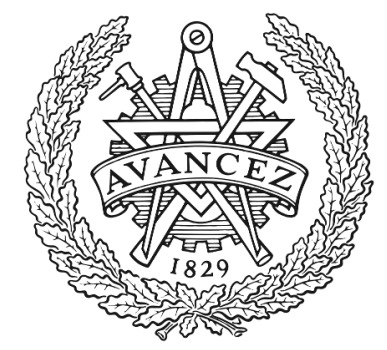

CHALMERS

UNIVERSITY OF TECHNOLOGY

\title{
Investigations of Tip Vortex Mitigation By Using Roughness
}

Downloaded from: https://research.chalmers.se, 2023-04-26 15:15 UTC

Citation for the original published paper (version of record):

Asnaghi, A., Svennberg, U., Gustafsson, R. et al (2020). Investigations of Tip Vortex Mitigation By Using Roughness. Physics of Fluids, 32(6). http://dx.doi.org/10.1063/5.0009622

N.B. When citing this work, cite the original published paper. 


\title{
Investigations of Tip Vortex Mitigation By Using Roughness
}

\author{
Abolfazl Asnaghi ${ }^{1}$, Urban Svennberg ${ }^{2}$, Robert Gustafsson ${ }^{2}$, and Rickard E. Bensow ${ }^{1, a}$ \\ ${ }^{1}$ Department of Mechanics and Maritime Sciences, Chalmers University of Technology, 41296 Gothenburg, Sweden \\ ${ }^{2}$ Kongsberg Hydrodynamic Research Centre, Kongsberg Maritime Sweden AB, 68195 Kristinehamn, Sweden \\ ${ }^{a}$ Author to whom correspondence should be addressed: rickard.bensow@chalmers.se
}

June 3, 2020

\section{Abstract}

The application of artificial roughness to mitigate tip vortex cavitation inception is analyzed through numerical and experimental investigations carried out on an elliptical foil. Different roughness configurations and sizes are tested and effects on cavitation inception, drag, and lift, are studied. Implicit Large Eddy Simulation (ILES) is employed to conduct the simulation on a proper grid resolution having the tip vortex spatial resolution as fine as $0.062 \mathrm{~mm}$. Two different approaches including using a rough wall function and resolving the flow around roughness elements are evaluated. New experiments, performed in the cavitation tunnel at Kongsberg Hydrodynamic Research Centre, for the rough foil are presented.

The vortical structures and vorticity magnitude distributions are employed to demonstrate how different roughness patterns and configurations contribute to the vortex roll-up and consequently on the tip vortex strength. It is found that the application of roughness on the leading edge, tip region and trailing edge of the suction side are acceptable to mitigate the tip vortex and also to limit the performance degradation. This is regarded to be in close relation with the way that the tip vortex forms in the studied operating condition. The analysis of boundary layer characteristics shows a separation line caused by roughness is the reason for a more even distribution of vorticity over the tip compared to the smooth foil condition leading to a reduction in vortex strength. For the optimum roughness pattern, both the numerical results and experimental measurements show a decrease in the tip vortex cavitation inception as large as $33 \%$ compared to the smooth foil condition with a drag force increase observed to be less than $2 \%$.

Keywords: Tip vortex, Mitigation, Roughness, Elliptical foil, LES. 


\section{Introduction}

There is a growing concern that underwater radiated noise (URN) introduced into the sea by human activities has detrimental effects on marine mammals. Among different URN sources, the most pervasive, yet more subtle, problem may be the increases in background noise levels from commercial shipping. Due to the low absorption rate of the oceans, low frequencies of URN travel miles away from their origin. Many sources of noise are located along with travelled paths in the sea, particularly in coastal and continental shelf waters, areas that often include important marine mammal habitats. Considering the worldwide shipping traffic, URN are known to affect the survivability of marine mammals by masking frequency bands used for their fundamental living activities $[1-3]$.

Ships URN can be classified into two categories according to their sources, machinery noise and flow-induced noise generated by the propeller rotation. Machinery noise is not strictly affected by the ship speed, and can be shielded by sound absorbing materials and vibration control. The flow-induced noise, however, is directly related to the ship speed. In general, when cavitation occurs on a propeller, the sound pressure level (SPL) increases considerably with the ship speed $[4,5]$. Therefore, there is a large difference between the SPL of a propeller operating in non-cavitating and cavitating conditions. As a result, the most important factor controlling the URN of a propeller is the cavity inception speed (CIS) [6].

Propeller cavitation can appear in many different forms, including tip vortex, sheet, cloud and supercavitation, depending on the propeller design and operating conditions. Among these forms, tip vortex cavitation (TVC) is usually the first type that appears on a propeller. This implies that TVC plays a key role in initiating an overall increasing SPL, and determining the URN [7]. Consequently, it is considered as the main controlled cavitation characteristics in the design procedure of low-noise propellers, where operating profile requires very low radiated noise emissions [8].

At present, there are various approaches to modify tip vortex structures in a way to delay or suppress the cavitation inception. Along with the common ways known from the classic propeller design such as the modification of profiles, pitch, and planform; some methods are developed specifically to affect the tip vortex region and to alter the tip vortex [9-13]. One of the promising passive methods to mitigate a tip vortex is the inclusion of roughness on the blade surfaces. Katz and Galdo [14] pointed out a distinct relation between surface roughness and tip vortex roll-up, demonstrating a shift in detachment point and a substantial reduction in tip vortex strength due to increasing the surface roughness. The blade surface roughness also contributes to the tip vortex properties. Johnsson and Ruttgerson [15] studied the influence of leading edge roughness on the tip vortex roll-up for different angles of attack. It is shown that application of roughness on the pressure side near the leading edge has a delaying effect on tip vortex cavitation, and leads to a substantial reduction in tip vortex strength. They also reported that applying the roughness can reduce the open water efficiency by $2 \%$. Later it was suggested by Philipp and Ninnemann [16] to apply the roughness only on a small area of the blade suction side close to the tip and trailing edge region, i.e. in the area of the tip vortex formation.

The turbulence generated by the roughness induced mixing in the shear layers around the vortex core, can destabilize the tip vortex and lead to its early breakdown [17]. By employing particle image velocimetry measurements, Ghimire and Bailey [18] investigated the effect of the free stream turbulence on the vortex decay. They showed that with the addition of free stream turbulence, the rate of decay of the peak tangential velocity of the vortex increases, whereas the rate of increase of core radius remains unchanged. Kruger et al. 
[19] reported that suction-sided sand grain roughness reduces the tip vortex strength and leads to pressure increase in the vortex core. Souders and Platzer [20] discussed that the thicker turbulent boundary layer on the wing tip results in an increased tip vortex core radius and a decrease in the tip vortex maximum tangential velocity as compared to the laminar flow case. They investigated this hypothesis by applying roughness on the tip region of a hydrofoil, and considered its contributions on the tip vortex characteristics. In order to avoid substantial increases in drag, the roughness was applied only to those areas of the foil tip whose streamlines contributed directly to the formation of the tip vortex. They reported an increase of TVC inception speed by $41 \%$ for pressure side roughness, $28 \%$ percent for suction side roughness, and $67 \%$ for pressure and suction side roughness.

Even though there have been several research studies, both numerically and experimentally, practical application of roughness to mitigate TVC inception has not been achieved yet. One possible reason for this is the lack of knowledge on the physics of tip vortex flows and how roughness interacts with the tip vortex. Most of the conducted studies have been either fully focused on experimental tests or on numerical analysis. The coexistence of the phase change and highly swirling flow creates a complex flow structure in the cavitating tip vortex regions which involve very small scale dynamics both in temporal and spatial coordinates [21-23]. This flow physics becomes even more challenging when the roughness is included [24]. Another limitation in roughness applications is the lack of study on the tip vortex mitigation using roughness on full scale applications. Most of the published studies and analysis are conducted on model scale cases without a proper attention on their extension into industrial cases. Experimental observations alone can show many of the phenomena occurring in the tip region but suffer from limitations in the measurements of all relevant flow features, e.g. velocity distributions and the pressure at the vortex core where cavitation inception occurs [25]. Advanced numerical simulations can provide further insights on the tip vortex properties but without validations and calibrations with experimental analysis, their outcomes become inconclusive. In this regard, both numerical and experimental investigations are employed in this study to provide further insights and knowledge on the TVC inception mitigation by using roughness.

In our previous studies, we employed computational fluid dynamics (CFD) and compared its results with high speed video recordings to evaluate the propeller tip geometry modification, as a classical way to mitigate TVC in the design stage [8]. The current paper extends the TVC inception analysis by including application of roughness. The main objective here is to evaluate and analyze the impact of roughness on formation of vortical structures, their interactions and effects on the main tip vortex, as well as on TVC mitigation. The analysis is conducted on an elliptical foil having the NACA $66_{2}-415$ cross section often used for experiments and analysis of tip vortex cavitation in the smooth surface condition [21,25-27]. The vortex structures around this type of foil resembles the propeller tip vortex behaviour while making it possible to be tested in more detail both experimentally and numerically. The simulations are conducted based on the numerical settings and modelling guideline, e.g. spatial mesh resolution and turbulence modelling that have been developed in our previous work [28].

As the roughness elements promote transition to turbulence in laminar boundary layers and alter the near-wall flow structures, they interfere with the tip vortex roll-up leading to a weaker tip vortex. However, this as well leads to a negative impact on the performance, i.e. having lower lift and higher drag forces, emphasizing the necessity of optimizing the areas covered by the roughness. Therefore, the overall focus of the current research is to find the most effective roughness size, and optimize the area where the roughness is applied in order 
to have a reasonable balance between the tip vortex mitigation and performance degradation. Based on the flow streamlines and vortical structures formed on the smooth foil, different sections of the foil surface on the suction side and pressure side are identified and their contribution to the tip vortex build up is analysed. Then, roughness is applied on different combinations of these sections to find the most optimum roughness area to mitigate the TVC. Similar to the suggestion by Souders and Platzer [20], roughness is applied only to those areas of the foil tip whose streamlines or vortical structures contribute directly to the formation of the tip vortex.

The generated spatial resolutions satisfy minimum requirements of tip vortex simulations using implicit LES in Open Source Field Operation and Manipulation (OpenFOAM) numerics, where at least 32 grid points are provided across the vortex diameter giving the spatial resolution as fine as $0.062 \mathrm{~mm}$. Two approaches are employed and compared to include the roughness effects: using a rough wall function and resolving the flow around the roughness elements [29,30]; these roughly correspond to an isotropic sand-grain roughness and a more sparse roughness distribution respectivly. In the modelling approach, the wall function is modified based on the non-dimensionalized roughness height to incorporate the roughness effects into the modelling. In one roughness pattern and in order to provide further insights about different methodologies of modelling roughness elements, the geometry of roughness elements is incorporated into the computational domain. As a result, two different computational domains having a similar cell resolution in the tip vortex trajectory but different wall normal resolutions are generated; $y^{+}=35$ prepared for modelling the roughness via the wall function and $y^{+}=3$ prepared for resolving the flow around the roughness elements. In both of the meshes, the baseline surface resolution on the foil has $x^{+}$and $z^{+}<250$, but is considerably finer in the refined tip region. To support the numerical simulations, a series of experimental tests are conducted by considering a few surface roughness configurations on the foil. These experiments are performed in the free surface cavitation tunnel at the Kongsberg Hydrodynamic Research Center (previously owned by Rolls-Royce), Kristinehamn, Sweden. We continue the paper by presenting the governing equations. Then, the elliptical foil geometry, computational domain, mesh specifications, and experimental set-up are briefly presented. The results start by the detailed description of the flow physics of smooth foil tip vortex flows to distinguish the foil surface regions effective in the tip vortex formation. Then, the results of the flow simulations around the fully rough foil are presented, including the evaluation of the roughness sizes. The numerical results of different roughness configurations are provided and compared with the experimental measurements where the distribution of vorticity magnitude is used as an indication of vortex roll-up and strength to evaluate different roughness configurations. Finally, the numerical results of wall modelled roughness and resolved flow around roughness elements are presented.

\section{Governing Equations}

The flow regime over a rough surface depends on how roughness elements interact with different parts of the boundary layer. If the roughness elements are embedded in the viscous sublayer, the friction drag is not affected by the roughness and the flow regime is smooth. In the case where the roughness elements heights are much larger than the boundary layer thickness, the fully rough regime forms where the viscous sublayer disappears and the drag significantly increases. In such a condition, wall skin friction and the roughness impact become independent of Reynolds number which means the viscous effect is no longer important; the drag increase is only due to pressure forces on the roughness elements. The transition regime is between these two regimes 
where both viscous and pressure forces on the roughness elements contribute to the drag.

The nondimensional roughness height used to characterize the flow regime can be represented by $\mathrm{K}_{\mathrm{s}}^{+}=u_{\tau} \mathrm{K}_{\mathrm{s}} / \nu$ where $K_{\mathrm{s}}$ is the roughness height, $u_{\tau}=\sqrt{\tau_{\mathrm{w}} / \rho}$ is the shear velocity, and $\tau_{\mathrm{w}}$ is the wall shear stress. In this study, we employ $\mathrm{K}_{\mathrm{s}}^{+}=2.5$ and $\mathrm{K}_{\mathrm{s}}^{+}=90$ as the limits of the transitional regime,

$$
\text { Flow regimes over rough surfaces }= \begin{cases}\mathrm{K}_{\mathrm{s}}^{+} \leq 2.5 & \text { smooth } \\ 2.5<\mathrm{K}_{\mathrm{s}}^{+}<90 & \text { transitionally rough } \\ 90 \leq \mathrm{K}_{\mathrm{s}}^{+} & \text {fully rough }\end{cases}
$$

The flow around the roughness elements can be either resolved or modelled. To resolve the flow, roughness geometries have to be included into the computational domain which obviously would demand for a higher computational resources. Modelling the flow around roughness elements requires much lower computational cells but as it involves simplification of the roughness geometry the flow physics may not correctly be modelled. In the current study, the wall function developed by Tapia (2009) for the inner region of the turbulent boundary layer, or the log-law region, (e.g. $11 \leq y^{+}$in OpenFOAM wall functions) is used,

$$
u^{+}=\frac{1}{\kappa} \ln \left(E \cdot y^{+}\right)-\Delta B
$$

with $\kappa=0.41, \mathrm{E}=9.8, y^{+}=u_{\tau} y / \nu$, and the velocity shift correction $\Delta B$ due to the roughness elements. As this model only affects the viscosity of the first cell adjacent to the wall, the height of the elements should be smaller than the height of the cells wall normal distance, i.e. $\mathrm{K}_{\mathrm{s}}^{+} \leq y^{+}$. Otherwise, the part of roughness elements that is located outside the adjacent cells will not be included in the modelling.

In a smooth regime represented by $\mathrm{K}_{\mathrm{s}}^{+} \leq 2.5$, the correction $\Delta B$ is set to zero and the wall function recalls the smooth wall function. For a transitionally rough regime where $2.5<\mathrm{K}_{\mathrm{s}}^{+}<90$, the correction reads,

$$
\Delta B=\frac{1}{\kappa} \ln \left[\frac{\mathrm{K}_{\mathrm{s}}^{+}-2.25}{87.75}+\mathrm{C}_{\mathrm{s}} \mathrm{K}_{\mathrm{s}}^{+}\right] \sin \left(0.425\left[\ln \left(\mathrm{K}_{\mathrm{s}}^{+}\right)-0.811\right]\right),
$$

where $C_{\mathrm{s}}$ is a constant representing shape and form of roughness elements. However, there is no clear guideline to adjust this coefficient. It is suggested that it varies from 0.5 to 1 where $\mathrm{C}_{\mathrm{s}}=0.5$ corresponds to the uniformly distributed sand grain roughness.

For a fully rough regime having $90 \leq \mathrm{K}_{\mathrm{s}}^{+}$, the $\Delta B$ correction is represented by,

$$
\Delta B=\frac{1}{\kappa} \ln \left[1+\mathrm{C}_{\mathrm{s}} \mathrm{K}_{\mathrm{s}}^{+}\right]
$$

The turbulent viscosity of cells adjacent to the rough wall is then recalculated using the following formula,

$$
\mu_{t}=\mu\left[\frac{y^{+} \kappa}{\ln \left(E y^{+} / e^{\kappa \Delta B}\right)}-1\right] .
$$

To model the flow field by using LES, the low pass filtered equations of mass and momentum are employed,

$$
\begin{gathered}
\frac{\partial \rho_{\mathrm{m}}}{\partial t}+\frac{\partial\left(\rho_{\mathrm{m}} \bar{u}_{i}\right)}{\partial x_{i}}=0 \\
\frac{\partial\left(\rho_{\mathrm{m}} \bar{u}_{i}\right)}{\partial t}+\frac{\partial\left(\rho_{\mathrm{m}} \bar{u}_{i} \bar{u}_{j}\right)}{\partial x_{j}}=-\frac{\partial \bar{p}}{\partial x_{i}}+\frac{\partial}{\partial x_{j}}\left(2 \mu \bar{S}_{i j}-B_{i j}\right)+\rho_{\mathrm{m}} g_{i}
\end{gathered}
$$


where the over bar denotes low pass filtered quantities, $B_{i j}=\rho\left(\overline{u_{i} u_{j}}-\overline{u_{i}} \overline{u_{j}}\right)$ is the subgrid stress tensor, $g_{i}$ is the body force, $\bar{u}_{i}$ and $\bar{p}$ are the velocity and pressure fields of the mixture flow, and $\rho_{\mathrm{m}}$ is the mixture density. The strain rate tensor, $S_{i j}$, is the symmetric part of the velocity gradient,

$$
\bar{S}_{i j}=\frac{1}{2}\left(\frac{\partial \bar{u}_{i}}{\partial x_{j}}+\frac{\partial \bar{u}_{j}}{\partial x_{i}}\right)
$$

In ILES, no explicit model is applied for $B_{i j}$, instead the numerical dissipation is considered enough to mimic the action of $B_{i j}$ [31-33]. Therefore, for fully resolved boundary layer mesh, $y^{+} \leq 5$, the dissipation of momentum convection scheme is the only source that mimics the turbulent viscosity. For wall-modelled ILES, the turbulent viscosity of the wall adjacent cells is recomputed by Eq. 5 .

In this study, the Q-criterion, which represents the local balance between shear strain and rotational tensor magnitudes, is employed to identify vortical structures in the flow [34,35],

$$
Q=\frac{1}{2}\left(\bar{\Omega}_{i j} \bar{\Omega}_{i j}-\bar{S}_{i j} \bar{S}_{i j}\right)
$$

where the vorticity is defined by,

$$
\bar{\Omega}_{i j}=\frac{1}{2}\left(\frac{\partial \bar{u}_{i}}{\partial x_{j}}-\frac{\partial \bar{u}_{j}}{\partial x_{i}}\right) .
$$

In order to evaluate the pressure field of the tip vortex, the cavitation number definition is employed,

$$
\sigma=\frac{p-p_{\mathrm{sat}}}{\frac{1}{2} \rho U_{\mathrm{ref}}^{2}}
$$

where $p_{\text {sat }}$ is the saturation pressure.

\section{Simluation set-up}

The tip vortex flow around an elliptical foil is selected as a test case in order to gain insight about the impact of roughness on the flow pattern and tip vortex properties. This type of elliptical foils is often used for experiments and analysis of tip vortex cavitation $[21,25,26,36,37]$. The geometry of the foil is an elliptical planform having the NACA $66_{2}-415$ cross section. Having NACA 6 series section introduces a low adverse pressure gradient over the foil and therefore longer laminar boundary layer.

In the previous studies conducted by the authors, the numerical requirements for accurately modelling the tip vortex formation on the smooth foil in the near field region have been evaluated $[28,38,39]$. The numerical case set-up here is thus continued based on the experimental study conducted in the cavitation tunnel in the Laboratory for Ship Hydrodynamics at Delft Technical University [26,36], and the results of the smooth foil are validated against these. The same set-up is used for the simulations with surface roughness but then compared with the new experiments described in the following section.

The tip geometry of the foil in different cross sections are presented in Fig.1. The trailing edge of the tested foil was truncated at a thickness of $0.3 \mathrm{~mm}$ due to manufacturing limitations where the root chord length after truncation is $\mathrm{C}_{0}=0.1256 \mathrm{~m}$. The projected surface area of the foil computed from the CAD file is $\mathrm{A}=0.01465 \mathrm{~m}^{2}$ where lift and drag coefficients are calculated based on this area, $\mathrm{C}_{\mathrm{l}}=\mathrm{L} /\left(0.5 \rho \mathrm{U}_{\text {inlet }}{ }^{2} A\right)$ and $\mathrm{C}_{\mathrm{d}}=\mathrm{D} /\left(0.5 \rho \mathrm{U}_{\text {inlet }}^{2} A\right)$. The foil has a half span of $150 \mathrm{~mm}$, so that the tip is positioned in the centre of the test section. The foil is also placed in the middle of the channel width where distance to each side is equal to $150 \mathrm{~mm}[26]$. 
(3)

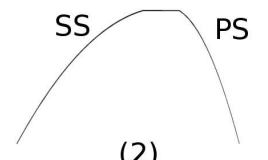

(2)

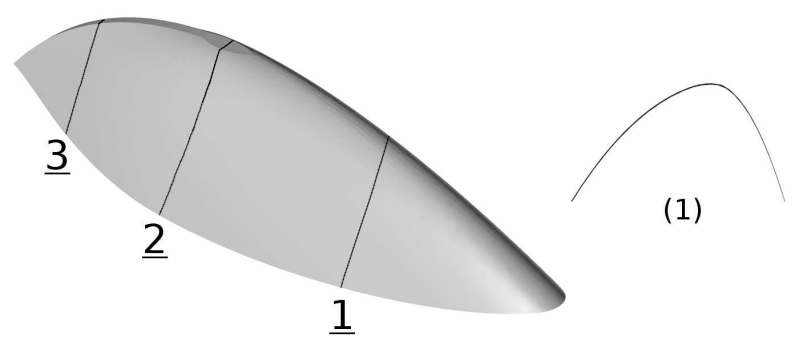

Fig. 1: Tip geometry at different cross sections of the foil, tip zoomed view, (1): a cross section in the leading edge part, (2): a cross section on the tip, (3): a cross section in the trailing edge part, PS: pressure side, SS: suction side.

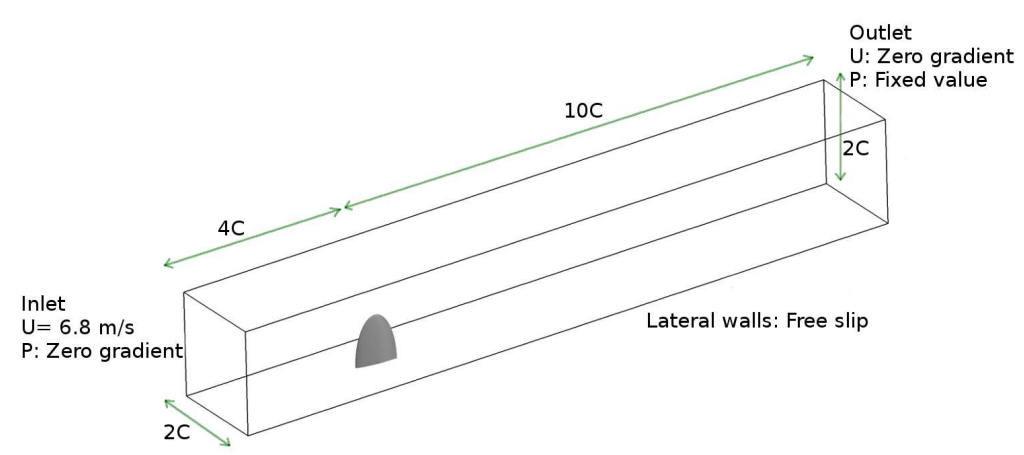

Fig. 2: Computational domain of the foil simulations.

In Fig. 2, the computational domain which mimics the Delft cavitation tunnel dimensions is presented. The simulations are conducted at the angle of attack equal to 9 degrees and a constant inlet velocity of $6.8 \mathrm{~m} / \mathrm{s}$ which corresponds to $\operatorname{Re}=8.95 \times 10^{5}$ based on the foil root chord length, $\mathrm{C}_{0}$. The outlet boundary is set as a fixed pressure boundary, and no-slip conditions are applied on the foil and bottom surfaces. The other boundaries are treated as slip conditions.

According to our findings, at least 32 grid points across the vortex diameter is required to predict a tip vortex flow in its near field region, i.e. 1.5 chord lengths downstream of the tip, using implicit LES in the OpenFOAM framework [28]. In Fig. 3, distribution of computational cells in the streamwise and in a cross section is presented. In this study, the tip vortex trajectory refinement is limited to 0.75 chord length downstream of the foil. An extra refinement box having the same resolution as the tip vortex refinement is added to refine the cells around the tip region, Fig. 3a.

Two different wall normal resolutions are considered. In Mesh (I) which is prepared for modelling the roughness via the wall function, the wall normal resolution is $y^{+}=35$, and in Mesh (II) which is prepared for resolving the flow around the roughness elements, the wall normal resolution is $y^{+}=3$. The prismatic layers in both mesh resolutions consist of 20 layers where different extrusion factors are used to provide a smooth transition between the prismatic layers and the volumetric cells outside the boundary layer. The baseline mesh resolution on the foil surface has both $x^{+}$and $z^{+}<250$, but considerably finer in the refined tip region. The baseline resolution is not sufficient for a proper wall-resolved LES, but the refined region is; it is thus expected that the 


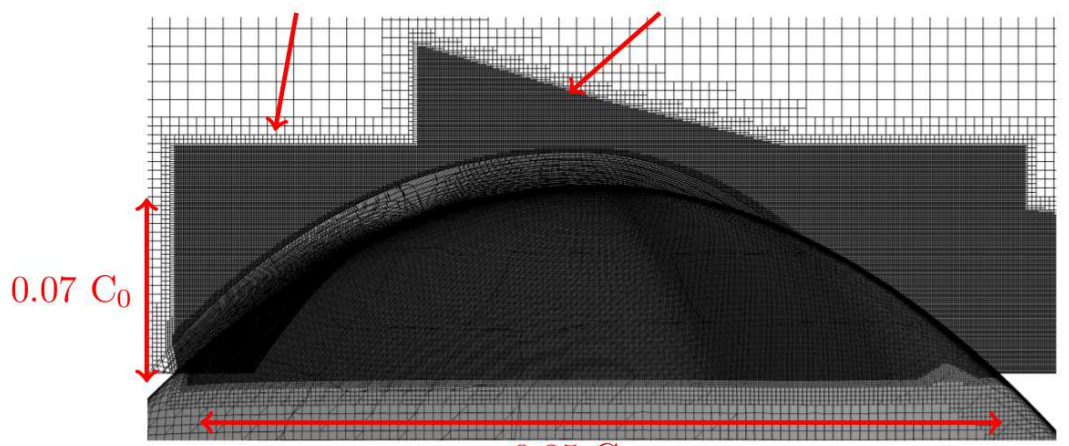

$0.35 \mathrm{C}_{0}$

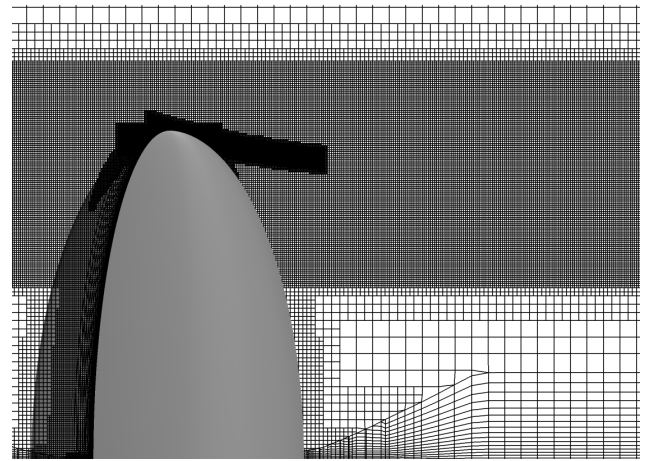

$\mathrm{b}$

Fig. 3: Mesh distribution in the streamwise and inplane directions. (a) Resolution around the tip, (b) Streamwise mesh distribution, and (c) Inplane mesh distribution.

vortex formation is correctly captured while the drag for the whole foil is somewhat underestimated.

Table 1: Mesh specifications of the elliptical foil.

\begin{tabular}{cccccc}
\hline Name & $y^{+}$ & $\begin{array}{c}\text { Total number } \\
\text { of Cells }(\mathrm{M})\end{array}$ & $\begin{array}{c}\text { In-plane cell } \\
\text { size }(\mathrm{mm})\end{array}$ & $\begin{array}{c}\text { Streamwise } \\
\text { cell size }(\mathrm{mm})\end{array}$ & $\begin{array}{c}\text { Number of cells in } \\
\text { the vortex core diameter }\end{array}$ \\
\hline Mesh (I) & 35 & 31.8 & 0.062 & 0.125 & 32 \\
\hline Mesh (II) & 3 & 35.4 & 0.062 & 0.125 & 32 \\
\hline
\end{tabular}

To include roughness elements in the Mesh (II), as a first step, the surface area where the roughness needs to be applied is selected. Then, a list of the surface mesh faces covering this area is created. Depending on the desired concentration of the roughness elements, a new list of randomly selected faces from the area original face list is created. In the next step, computational cells that are in a certain distance, here the roughness elements height, of the randomly selected faces are removed from the computational domain. The advantage of this approach is its simplicity and capability to apply random roughness elements. However, as it is applied on the original mesh resolution, the number of cells surrounding the roughness elements are restricted by the resolution of the original model.

The resolving flow around roughness elements is limited to the optimum roughness height obtained in the section 6.2 and also to the optimum roughness area derived in the section 6.3. As the surface resolution at the tip region 


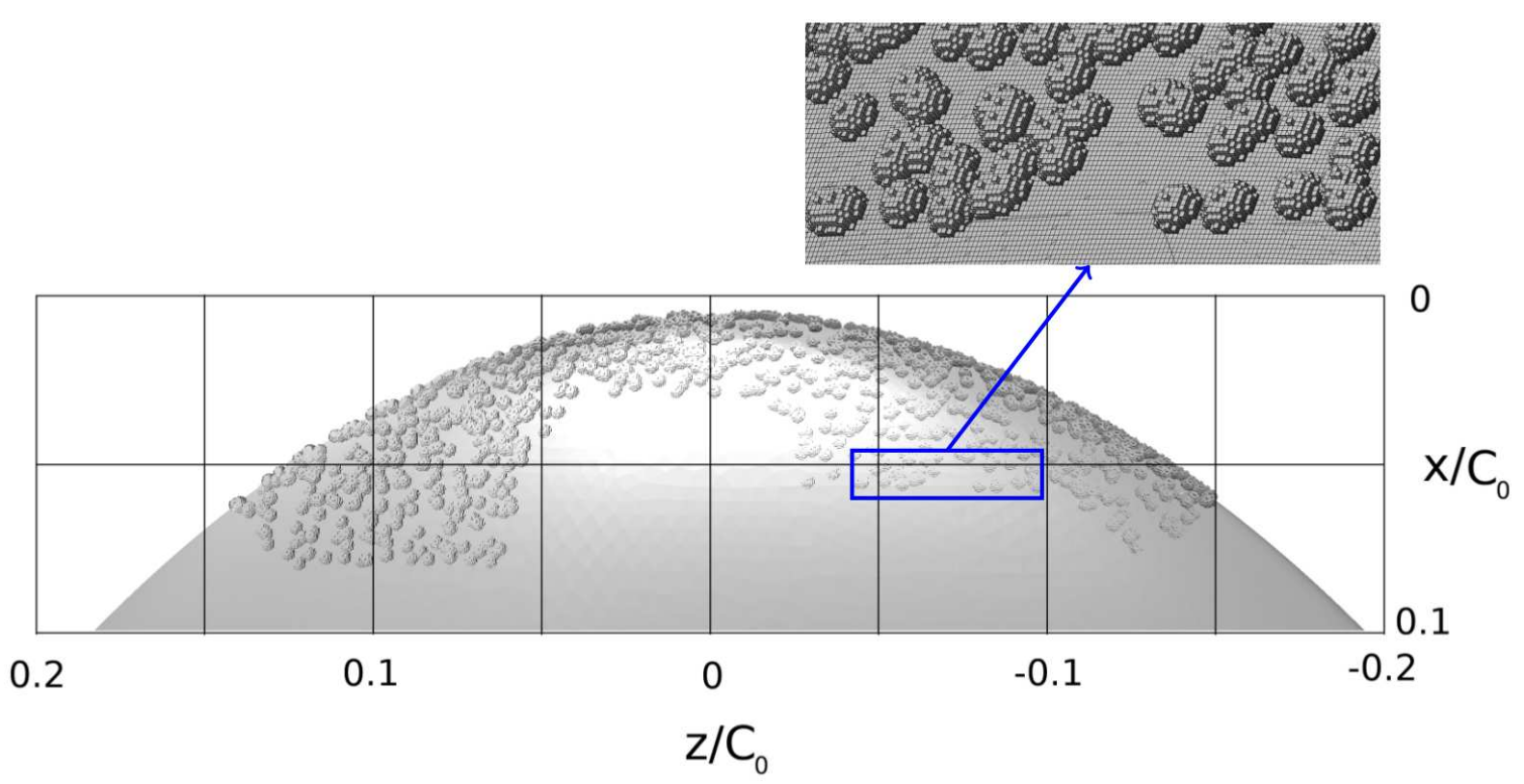

Fig. 4: Roughness elements distribution in Mesh (II).

is much finer than that the considered roughness height, each roughness element covers around 8 surface faces. The number of randomly selected faces is chosen in a way to roughly lead to roughness elements concentrations around 50 \%. In Fig. 4, the roughness elements distribution is presented. The close-up view can clearly show how the roughness elements are randomly distributed and how their surrounding resolutions are limited by the original mesh resolution.

The OpenFOAM package, used in this study for numerical simulations, is an open source code written in $\mathrm{C}++$ to model and simulate fluid dynamics and continuum mechanics [40]. Converted meshes from StarCCM+ to OpenFOAM generally contain some highly skewed faces, and also show high non-orthogonality between neighbouring cells. These poor quality cells usually appear either on the interface of the prismatic boundary layer and the volumetric cells, or at the interface of the refinement regions and volumetric cells. The consequences are stability issues, and therefore limitation in using high order schemes. Therefore, the divergence terms are computed by employing a vector based TVD limited second order scheme (limitedLinearV). The other face properties are interpolated by the second order linear scheme. All of the surface gradients have been corrected to consider non-orthogonality of the computational cells. This combination of schemes has previously been validated for ILES of tip vortex flows [41].

\section{Experimental tests setup}

As the available experimental measurements for this elliptical foil in open literature are only reported for smooth surface, a series of experimental tests are conducted by considering a few surface roughness configurations on the foil. These experiments are performed in the free surface cavitation tunnel at the Kongsberg Hydrodynamic Research Center, Kristinehamn, Sweden. The cross section of the tunnel test section is $0.8 \times 0.8 \mathrm{~m}^{2}$ at the inlet and $0.8 \times 0.82 \mathrm{~m}^{2}$ at the outlet. The vertical direction was extended gradually from inlet to outlet, to compensate for the growth of the boundary layer, in order to facilitate a nearly zero-pressure gradient in the streamwise direction. This is provided by having the top plate horizontal and the bottom plate with a downward 
slope of 0.4 degrees where at the foil section, the vertical distance is about $0.81 \mathrm{~m}$.

In order to test on a wider range of operating conditions, the experimental measurements are conducted on the foil having a uniform geometrical scale ratio of 2.398 compared to the elliptical foil tested at TU Delft $[26,27]$. This leads to the root chord length $\mathrm{C}_{0}=301.2 \mathrm{~mm}$ and the span length of $\mathrm{S}=360 \mathrm{~mm}$ in the tested foil. The measurements are performed with respect to the averaged free stream velocity ranging from $2.84 \mathrm{~m} / \mathrm{s}$ to $4 \mathrm{~m} / \mathrm{s}$ with typical fluctuations of $\pm 0.7 \%$. The operating conditions were monitored during each experiment to prevent any deviations during the measurements. The water temperature is measured with a PT-100 sensor placed submerged in the tunnel water downstream of the test section. The dissolved oxygen concentration (DO) was used as a measure of the amount of dissolved gas in the water using a fluorescence-based optical sensor (Trioxmatic 690). It has a range of 0-600\% which equals to 0-60 $\frac{\mathrm{mg}}{\mathrm{l}}$ and the measurements were conducted at around $40 \%$ corresponding to $4 \frac{\mathrm{mg}}{\mathrm{l}}$. As only dissolved oxygen is measured, this is taken as a representative indicator for the total amount of dissolved gas.

The cavitation tunnel static pressure is measured with a low pass filter at about $0.2 \mathrm{~Hz}$ with a digital absolute pressure sensor (Rosemount 3051S). The pressure is measured in the air volume and compensated for the water depth down to the foil tip. The typical accuracy is $0.05 \%$ of full-scale pressure $\left(1.1 \times 10^{5} \mathrm{~Pa}\right)$, which is $55 \mathrm{~Pa}$. The tunnel free stream velocity is measured using a pitot tube having the same type of the pressure transducers and located upstream of the wing. The measurements are conducted at the angle of attack (AOA) equal to 9 degrees where the inlet velocity is kept constant and the cavitation number is adjusted by changing the outlet pressure of the channel. Due to resource limitations, the roughness experimental tests are focused only on the roughness patterns, e.g. Fig. 5, determined through the CFD analysis with the roughness height equal to 230 $\mu \mathrm{m}$.

As presented in Fig. 5, different methods of applying roughness on the foil surface are investigated in the experimental tests. In one approach, roughness elements are applied uniformly on the surface creating a uniform rough patch. This provides a suitable case for comparison with wall-modelled roughness of the numerical results. The second approach applies roughness elements locally, more like randomly placed larger roughness elements. This corresponds to the resolved roughness flow numerical results.

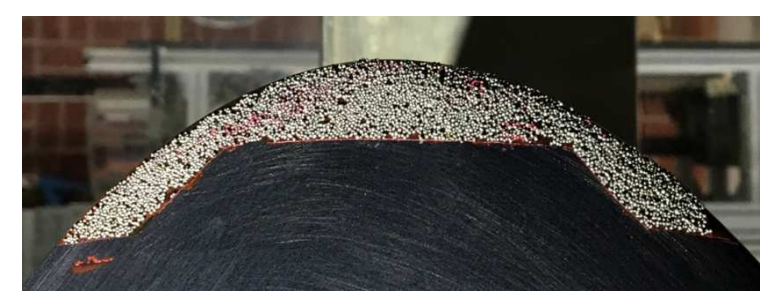

a

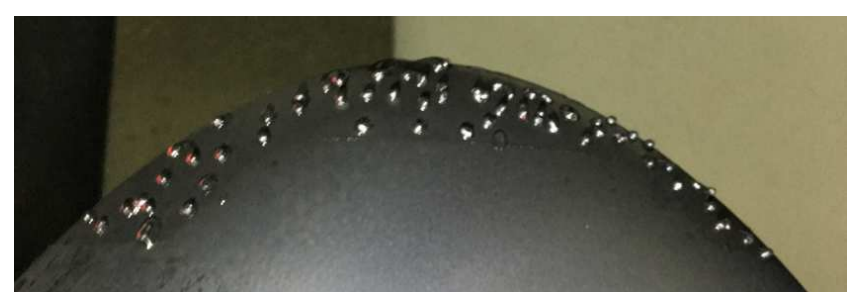

b

Fig. 5: Distributions of sand grains on the foil suction side, tip zoomed view. (a) Uniform distributions,(b) Sparse distributions.

\section{Results}

Formation of a tip vortex on a wing with finite span depends on the boundary layer development on both suction side and pressure side. Interactions of these two boundary layers and then the trailing vortices determine the tip 
vortex strength. As a result, tip vortex mitigation demands for identifications of key parameters in tip vortex formation and their origin; it should be defined which regions provide and feed momentum into the tip vortex. Then, the main goal of tip vortex mitigation is to apply roughness in these regions in a way to interrupt and disturb the tip vortex momentum delivery without a significant performance degradation.

\subsection{Smooth foil}

The first part of the results is to analyze the tip vortex flow in order to identify regions that provide the tip vortex momentum. In this regard, distribution of vortical structures and flow streamlines over the smooth foil are considered. To distinguish the streamlines that convey the tip vortex momentum, the streamlines passing through the vortex region are emphasized. The tip vortex region is defined as the solid body rotation vortex core part, $\mathrm{r} / \mathrm{r}_{\mathrm{v}}<1$, and its outer part where the flow tangential velocity is still effective [42]. Vortical structures are presented by $\mathrm{Q}$ iso-surfaces where the focus is on structures that contribute to the low pressure region of the tip vortex.

In Fig. 6, the flow streamlines that pass through the tip vortex region at the section $\mathrm{z} / \mathrm{C}_{0}=0.4$ downstream of the foil are presented. The streamlines are plotted separately for the flow that goes inside the vortex core, black lines, and for the flow that forms the outer region around the vortex core, white lines.

The figure clarifies that the leading edge and the region very close to the tip on both suction side and pressure side are effective regions in the tip vortex development. It is observed that most of the flow streamlines of the suction side leading edge form the tip vortex core rather than its outer region while the pressure side streamlines seem to feed both the vortex core and the outer region. As in the studied operating condition the tip vortex is formed on the suction side, the pressure side tip region seems to be less effective in feeding momentum into the vortex core.
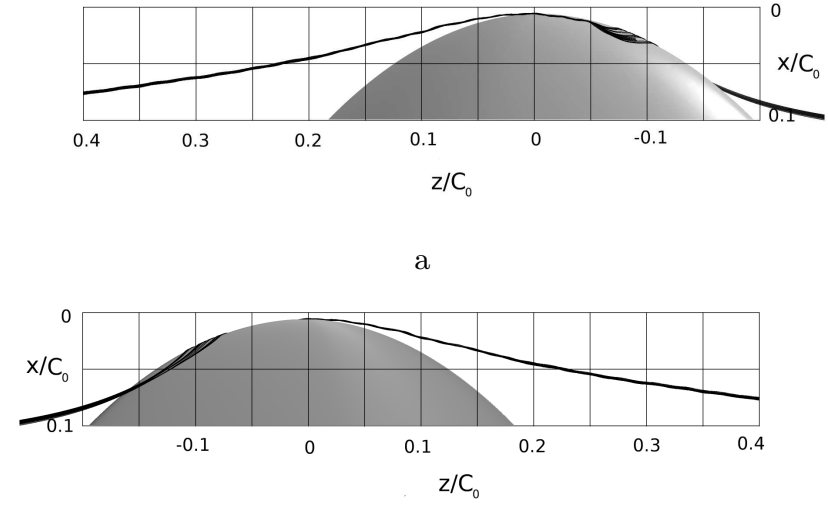

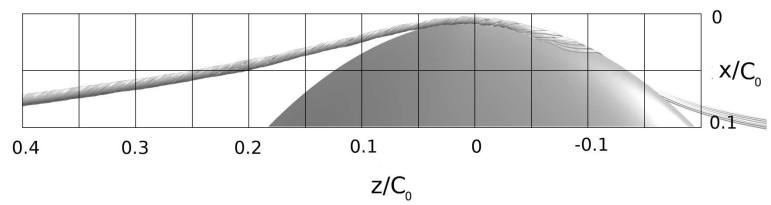

b

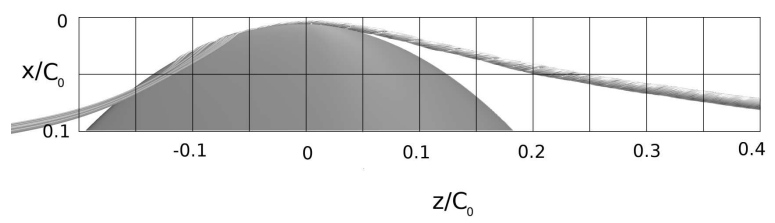

d

Fig. 6: Flow streamlines passing through the vortex core and its outer region at the section $\mathrm{z} / \mathrm{C}_{0}=0.4$, smooth foil. (a) Vortex core streamlines, suction side, (b) Outer region streamlines, suction side, (c) Vortex core streamlines, pressure side, (d) Outer region streamlines, pressure side.

The distribution of vortical structures, Fig. 7, indicates a very little contribution from the pressure side, only in a small region on the top of the foil. On the suction side, vortical structures are formed mostly on the leading edge, a region close to the tip that covers $(0,-0.05)<\left(\mathrm{x} / \mathrm{C}_{0}, \mathrm{z} / \mathrm{C}_{0}\right)<(0.05,0.1)$, and around the trailing edge. The trailing vortices are generated in the region located at $(0.05,0.1)<\left(\mathrm{x} / \mathrm{C}_{0}, \mathrm{z} / \mathrm{C}_{0}\right)<(0.1,0.2)$. These 
vortices are basically interactive with the low-pressure region of the tip vortex where the minimum tip vortex pressure usually occurs, i.e. $0.1<\mathrm{z} / \mathrm{C}_{0}<0.2$.
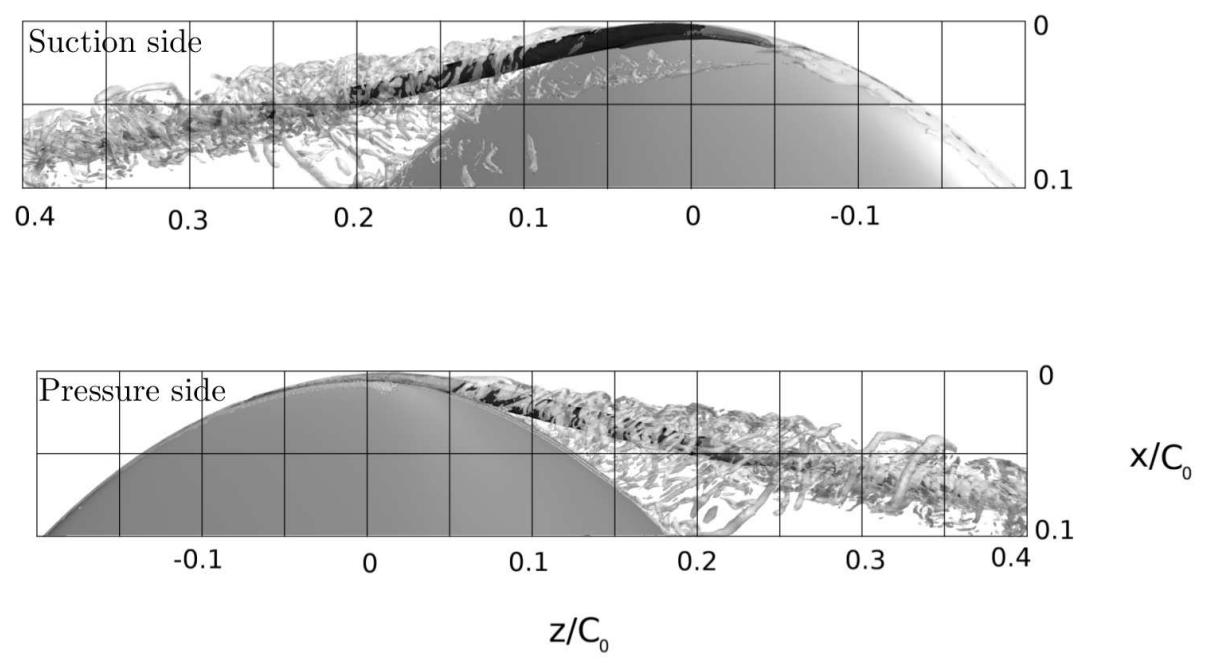

Fig. 7: Distribution of the iso-surface $\mathrm{Q}=800$ on the suction side and pressure side, smooth foil. The tip vortex is presented by averaged pressure iso-surface corresponding to $\mathrm{C}_{\mathrm{p}}=-2.4$ in black colour.

Based on the discussion provided, it can be concluded that the main effective areas on the tip vortex formation are the leading edge and the top region close to the tip of both sides, and the trailing region of the suction side. The selected areas based on results and discussions provided for Fig. 6 and 7 are described in Fig. 8. The figure only includes the suction side view, but the same area description on the pressure side is used.

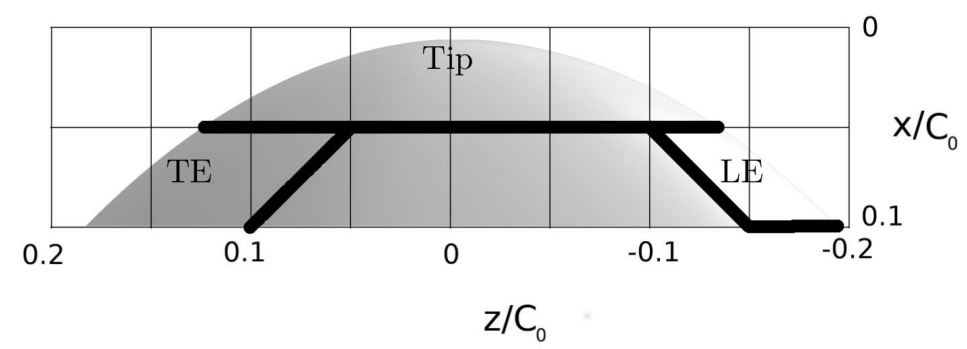

Fig. 8: Effective areas of the suction side in formation of tip vortex; leading edge (LE), trailing edge (TE).

\subsection{Fully rough foil}

We start the analysis of the roughness impact on the TVCI by examining a fully rough foil. In such a condition, it is assumed both sides of the foil are covered by a uniformly distributed sand grains and is modelled using the wall function approach. Then, the roughness height is varied to find the most optimum height in the current operating condition. In this regard, cavitation inception based on the minimum pressure is employed as a criterion to compare different roughness heights ranging from $10 \mu \mathrm{m}$ to $500 \mu \mathrm{m}$. The roughness sizes are selected in a way to be large enough to provide substantial increases in the tip boundary layer thickness, but also small enough to avoid local surface cavitation as recommended by [20].

As it is expected, roughness application leads to a weaker tip vortex compared to the one observed in the smooth foil condition, and therefore delays TVCI, Fig. 9. This comes at the cost of drag increase as roughness 
increases the turbulent viscosity on the foil surface. By increasing the roughness height, cavitation inception, which represents the strength of the tip vortex, decreases. However, after a certain value, $250 \mu \mathrm{m}$ in the current study, further increasing the roughness height does not affect the tip vortex mitigation but leads to higher drag forces. Consequently, it is concluded that roughness height equal to $250 \mu \mathrm{m}$ is the optimum height for the current operating condition.

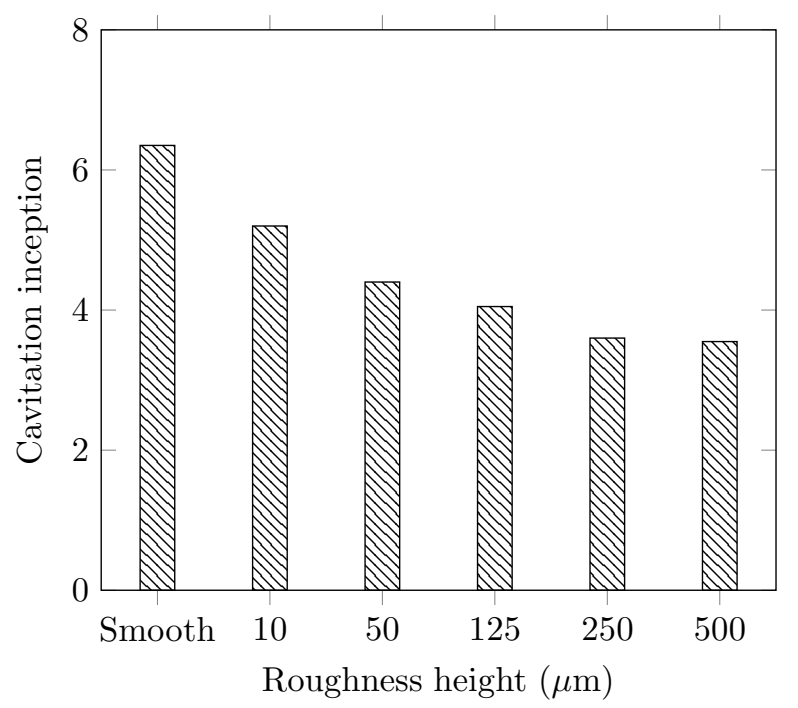

Fig. 9: Variation of the cavitation inception versus the surface roughness height for the fully rough foil.

As an example, illustrated in Fig. 10, the flow properties of the smooth foil and the fully rough foil having $\mathrm{K}_{s}=250 \mu \mathrm{m}$ are compared. The flow streamlines presented by white lines are limited to those streamlines passing through the vortex core in the smooth condition at the section $\mathrm{z} / \mathrm{C}_{0}=0.4$, similar to Fig. 6a. The foil surface is coloured by the averaged turbulent viscosity, and the tip vortex is presented by the iso-surface of pressure corresponding to $\mathrm{C}_{\mathrm{p}}=-3.2$ in grey colour. As can be observed, the distribution of the turbulent viscosity on the foil indicates a region with very high turbulent viscosity in the tip region of the fully rough condition that change the boundary layer and pressure distribution, and consequently the flow streamlines, over the foil. In the smooth surface condition, the streamlines are concentrated at the tip and form a leading edge vortex which initiates the tip vortex approximately at the section $\mathrm{z} / \mathrm{C}_{0}=-0.05$. In the fully rough condition, the flow streamlines are distributed more evenly on the tip, and therefore, are not concentrated or strong enough to form the tip vortex right on the tip of the foil. As a result, in the smooth condition, the streamlines provide the momentum of the tip vortex core while in the fully rough condition they rotate around the core and do not contribute to the vortex core momentum.

\subsection{Roughness area optimization}

To find a balance between the TVC mitigation and performance degradation, the roughness area optimization is limited to the regions that are highlighted in the smooth foil analysis, Fig. 8. Different cases containing different combinations of these roughness areas are constructed, Table 2. Evaluation of these combinations not only provides the possibility to find an optimum roughened pattern, but also gives the chance to analyse the flow physics of boundary layers at different regions around the foil and their interaction with tip vortex formation and development. In this evaluation, the roughness area is assumed to be uniform, i.e. wall functions 

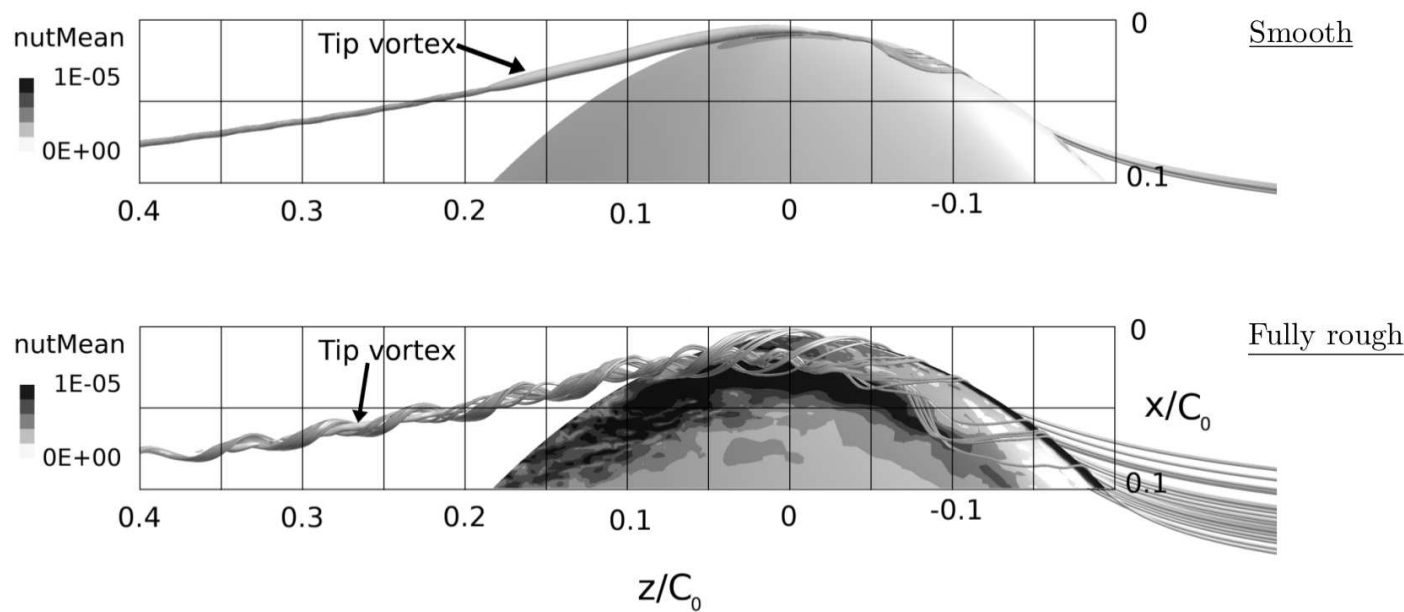

Fig. 10: Suction side view of the vortex core streamlines (while lines) for the smooth and rough surface $\left(\mathrm{K}_{s}=250\right.$ $\mu \mathrm{m})$. The foil surface is coloured by the turbulent viscosity and the tip vortex is represented by the iso surface of $\mathrm{C}_{\mathrm{p}}=-3.2$ in grey colour.

with $\mathrm{C}_{\mathrm{s}}=0.5$ in Eq. 3 and 4 are used, with the average roughness height equal to $250 \mu \mathrm{m}$.

Table 2: Case description of different roughness areas; SS: suction side, PS: pressure side, LE: leading edge, TE: trailing edge.

\begin{tabular}{|c|c|c|c|c|c|}
\hline Case & $(\mathrm{I})$ & (II) & (III) & (IV) & $(\mathrm{V})$ \\
\hline Roughness & SS Tip & PS Tip & SS Tip and LE & SS Tip & SS Tip, LE and TE \\
areas & SS LE & PS LE & PS Tip and LE & SS LE & PS Tip and LE \\
\hline
\end{tabular}

In Table 3, the lift and drag forces of these cases are presented where the values are normalized by the smooth foil forces. The experimental measurements of cases (I) and (IV) are also included in this table. The results of the FR foil is also included in this table as the reference of the extreme performance degradation condition. As discussed before, it is observed that having roughness all over the foil increases the drag force by $85.7 \%$ and decreases the lift force by $6.51 \%$. The predicted force variations of the limited roughness area cases show a much lower negative impact of the roughness on the performance. Also, it can be noted that having roughness on the pressure side, i.e. case (II),(III), and (V), contributes more to the performance degradation. Among the cases, the highest increased drag force is for case (III) where the roughness is applied on the leading edge and tip of the suction side and pressure side. Inclusion of roughness on the suction side trailing edge, i.e. case (V), somehow reduces the negative impact of the roughness on the forces. The results clarify that having roughness only on the suction side, case (I) and (IV), are favourable in terms of the forces evaluation of the roughness impact. The comparison with the experimental measurements shows a reasonably good agreement, and also indicates that the performance degradation is less than $1.44 \%$ in the optimized roughness area.

The predicted cavitation inception with different roughness patterns is presented in Fig. 11 along with the experimental measurements. Among the studied cases, the lowest cavitation inception points occur for the case (V), and then case (IV). However, as the performance degradation is much lower in case (IV), this case is considered as the outcome of the roughness area optimization. It is noted that when the roughness is applied 
Table 3: Percentage of the drag and lift forces variations relative to the smooth foil condition. Numerical results are averaged ILES, $\alpha=9^{\circ}$, roughness height $=250 \mu \mathrm{m}, \operatorname{Re}=8.95 \times 10^{5}$; Experimental measurements conditions: $\alpha=9^{\circ}$, roughness height $=230 \mu \mathrm{m}, \operatorname{Re}=8.55 \times 10^{5}$.

\begin{tabular}{|c|cc|cc|}
\hline \multirow{2}{*}{ Case } & \multicolumn{2}{|c|}{ Numerical results } & \multicolumn{2}{c|}{ Experimental measurements } \\
\cline { 2 - 5 } & Drag force(\%) & Lift force(\%) & Drag force(\%) & Lift force(\%) \\
\hline (I) & 0.8 & -0.04 & 1.44 & 0.27 \\
(II) & 10.5 & -0.93 & - & - \\
(III) & 16.9 & -1.51 & - & - \\
(IV) & 1.7 & -0.07 & 1.0 & -0.24 \\
(V) & 9.0 & -0.81 & - & - \\
\hline FR & 85.7 & -6.51 & - & - \\
\hline
\end{tabular}

only on the leading edge and tip of the pressure side, the TVCI is not reduced, i.e. case (II). This indicates the necessity of having roughness on the side where the tip vortex forms, e.g. suction side in the evaluated operating condition. During the experimental tests, the inception point is determined through visual observations of cavitation appearance in the tip vortex region obtained from high-speed recordings. The condition where the expanded bubble inside the tip vortex can be detected by a naked eye is considered as an inception point. The comparison between the numerical results (bars with dashed lines) and the experimental measurements (bars with the solid colour) shows a very good agreement. It clearly highlights the capability of the current method in modelling the roughness and its impact on the tip vortex cavitation.

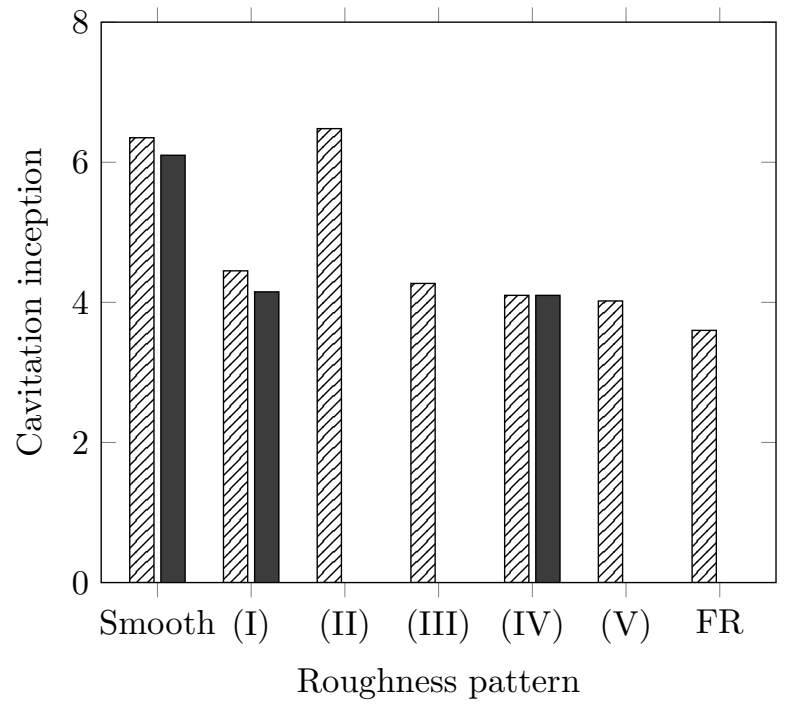

Fig. 11: Variation of the cavitation inception versus different surface roughness areas, FR: fully rough foil, roughness height $=250 \mu \mathrm{m}$, solid bars (smooth, (I) and (IV)) are the experimental measurements.

In Fig. 12, the vorticity magnitude distribution at $\mathrm{z} / \mathrm{C}_{0}=0$ as an indicator of vortex formation state is presented for different roughness arrangements. The figure includes the zoomed tip view in the free stream direction where the dimensions are normalized by the root chord length. The comparison clearly shows that in the smooth foil condition, Fig. 12a, the formation of the tip vortex has the largest high-vorticity region located close to the tip at the suction side, almost at $\left(\mathrm{x} / \mathrm{C}_{0}, \mathrm{y} / \mathrm{C}_{0}\right)=(0,0.01)$. In case (II) where the roughness is applied only on 
the pressure side, the region covering the tip vortex center is slightly shifted downward on the suction side while the strength of the vorticity magnitude remains very similar to the smooth foil condition. Application of roughness on the suction side, however, has a larger impact on the tip vortex region and its strength. As can be observed from cases (I), (IV) and (V), the tip vortex strength has been reduced significantly compared to the smooth foil condition, and has been moved further downward to $\left(\mathrm{x} / \mathrm{C}_{0}, \mathrm{y} / \mathrm{C}_{0}\right)=(0.01,0.02)$. The displacement of the tip vortex region downward indicates that the momentum has to be distributed over a larger area leading to a smaller high momentum magnitude region compared to the smooth condition. The vorticity distributions of case (I) and case (IV) at this section, Fig. 12b and Fig. 12d, are found to be similar. This clarifies that roughness application on the trailing edge area has a little impact on the tip vortex formation.

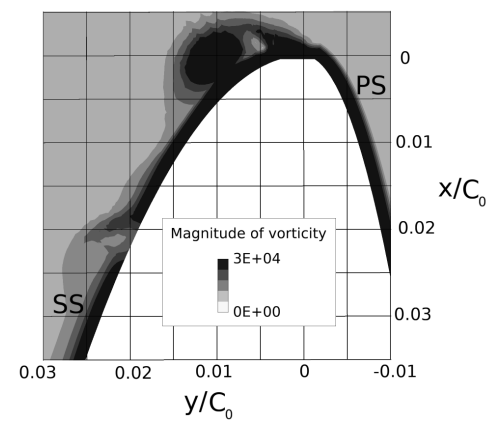

a

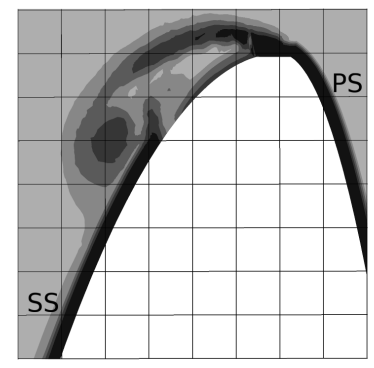

d

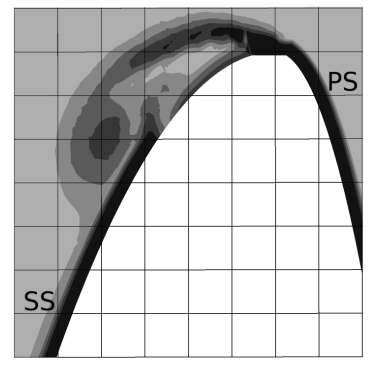

$\mathrm{b}$

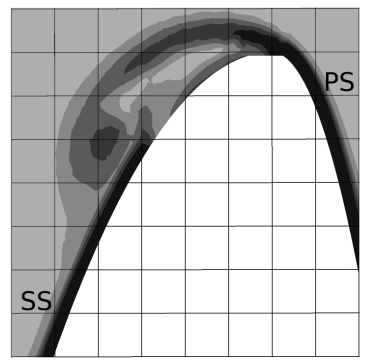

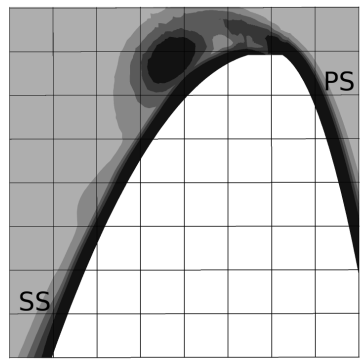

C

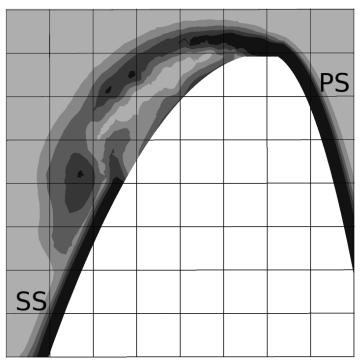

f

Fig. 12: Vorticity magnitude distribution at $\mathrm{z} / \mathrm{C}_{0}=0$, SS: suction side, PS: pressure side. (a) Smooth, (b) Case (I), (c) Case (II), (d) Case (IV), (e) Case (V), (f) Fully rough.

While the boundary layer separation on the suction side in the smooth foil happens at $\left(\mathrm{x} / \mathrm{C}_{0}, \mathrm{y} / \mathrm{C}_{0}\right)=(0.0225,0.02)$, Fig. 12a, for the suction side roughness application presented in cases (I), (IV) and (V) it is moved further upward happening at $\left(\mathrm{x} / \mathrm{C}_{0}, \mathrm{y} / \mathrm{C}_{0}\right)=(0.0075,0.0125)$. The flow streamlines over the foil indicates that in the roughness application two separation lines located at the leading edge and the tip regions of the suction side are observed, Fig. 13b and Fig. 13c. The separation line on the leading edge of the smooth condition, Fig. 13a, is noted to happen later compared to the rough condition.

Fig. 14 includes the vorticity magnitude distributions of different roughness arrangements at $\mathrm{z} / \mathrm{C}_{0}=0.1$. As expected, the smooth condition has the largest high vorticity magnitude region compared to other cases, Fig. 14a. For this case, the vorticity is well concentrated around the vortex center indicating the largest vortex roll-up among other cases. While the impact of pressure side roughness is not visible at section $\mathrm{z} / \mathrm{C}_{0}=0$, Fig. 12c, its impact on the vortex roll-up can be highlighted by comparison between Fig. 14a and Fig. 14c. The difference between case (I) and case (IV) highlights the impact of the trailing edge roughness on the vortex 


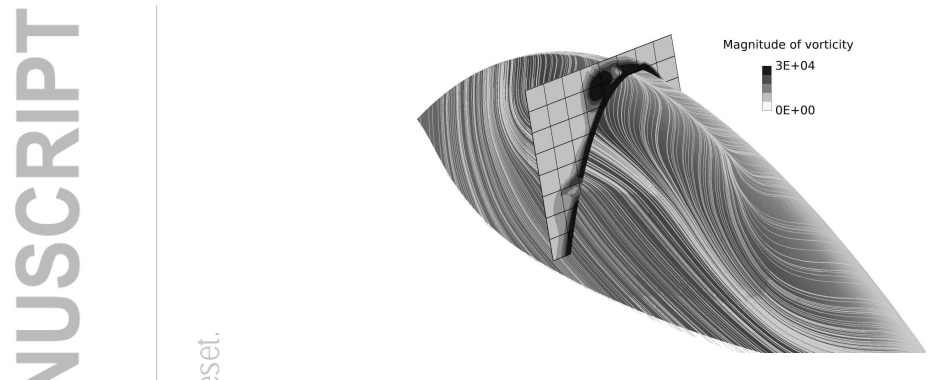

a

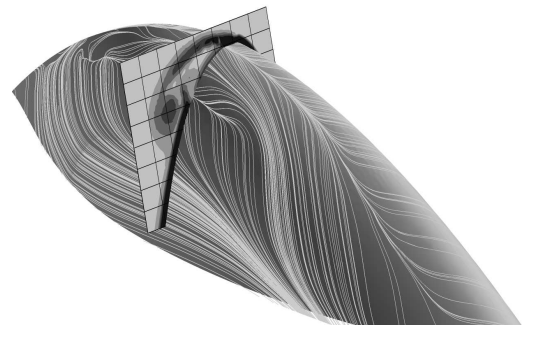

$\mathrm{b}$

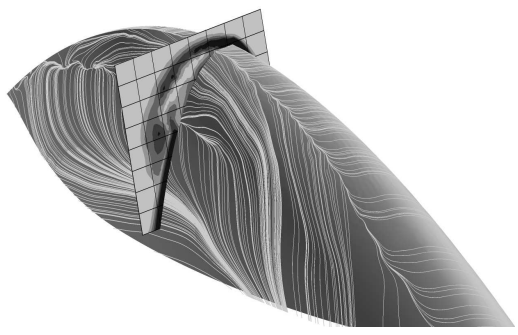

Fig. 13: Flow streamlines over the foil surface along with the vorticity magnitude distribution at $\mathrm{z} / \mathrm{C}_{0}=0$, isometric view of the suction side. (a) Smooth, (b) Case (IV), (c) Fully rough.

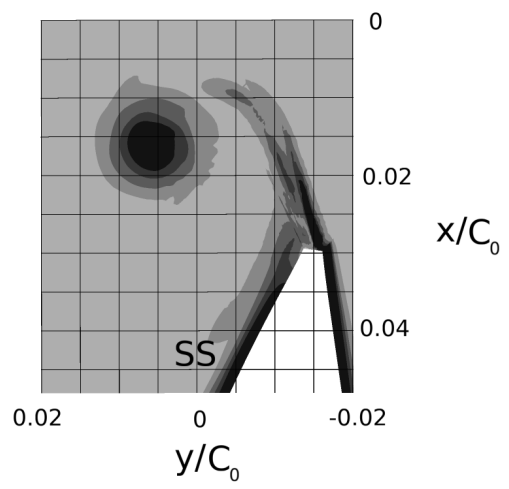

a

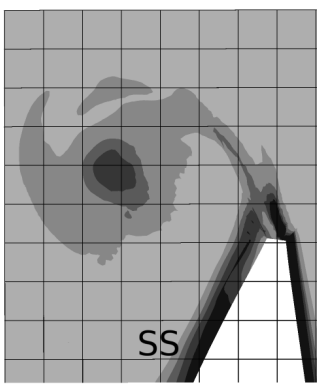

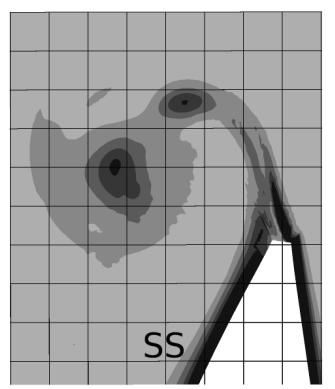

b

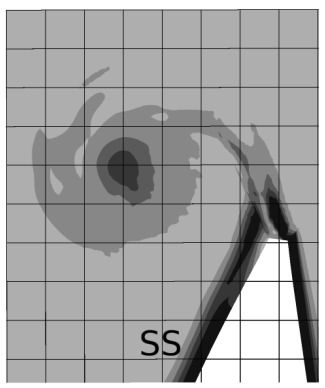

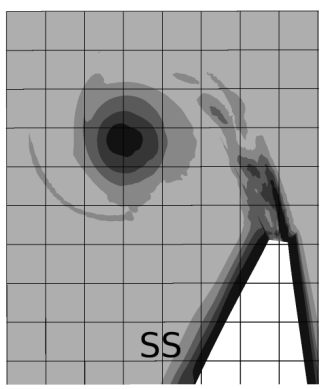

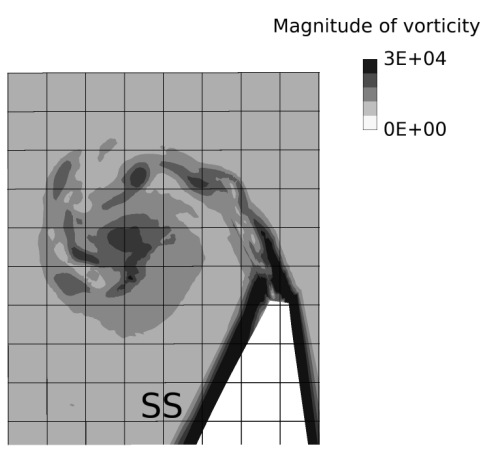

d

f

Fig. 14: Vorticity magnitude distribution at $\mathrm{z} / \mathrm{C}_{0}=0.1$, SS: suction side. (a) Smooth, (b) Case (I), (c) Case (II), (d) Case (IV), (e) Case (V), (f) Fully rough.

roll-up and tip vortex development. As noted from Fig. 12b and Fig. 12d, the vorticity distribution of these two cases at the vortex formation state, e.g. at $\mathrm{z} / \mathrm{C}_{0}=0$, is similar. However, further downstream of the tip where the trailing vortices roll-up impact are important, e.g. $\mathrm{z} / \mathrm{C}_{0}=0.1$, the difference between these two cases becomes more visible, Fig. 14b and Fig. 14d. In case (IV) where the roughness is applied on the trailing area as well, the strength of the tip vortex is weaker compared to the case (I) indicating lower trailing vortices roll-up strength in this case. Further inclusion of roughness on the pressure side, comparison of case (IV) and (V), only 
slightly weakens the tip vortex. Among the studied cases, the fully rough condition presented in Fig. 14f shows the most distributed and scattered high vorticity magnitude regions indicating that the flow consists of many small vortical structures interacting with each other. This prevents formation of a single strong tip vortex and consequently leads to the most suppressed tip vortex cavitation inception. 


\subsection{Physics of tip vortex development}

A detailed investigation of the near tip region can determine the relation between boundary layer (BL) characteristics and vortex roll-up in the smooth and roughened cases. For the studied foil, the tip geometry consists of a rounded tip in the leading edge part, i.e. $\mathrm{z} / \mathrm{C}_{0}<0$, and a truncated section in the aft part, Fig.1. The truncated section acts similar to a bluff body forcing a clear flow separation at the trailing edges where the flow discontinuity happens. The form of the BL development in the leading edge part, however, depends on the load distribution and the pressure difference between the pressure and suction sides of the foil. In the previous study of the authors [43], it is presented that the BL development on the foil and specifically the BL separation line, contributes significantly to the vortex core formation and its characteristics. Here, it will be discussed how these characteristics differ between smooth and roughened cases.

In Fig. 15, the flow streamlines for the smooth and roughness pattern (IV) are presented. The comparison of the flow streamlines on the pressure side shows an insignificant difference between the two cases. In both conditions, a laminar BL development and a mid-chord separation line maintaining a constant relative chordwise position in approximately 72 percent of the local chord length are observed. These results are in good agreement with the experimental oil flow visualisations conducted by Maines and Arndt [44] where they reported a similar pressure side separation line occurring in approximately 70 percent of the local chord length. More interestingly, it is also observed that the pressure side characteristics of BL are less sensitive to variation of angles of attack and load conditions [45]. This clarifies that the tip vortex strength reduction observed in roughened cases is not mainly because of the cross flow momentum transfer change from the pressure side into the suction side, but rather is a result of how this momentum has been distributed around the tip vortex in the suction side of roughened cases.

Looking now to the suction side, the BL forward of the separation line remains almost two-dimensional out to the tip. This is believed to be related to a favourable pressure gradient developed over the foil to approximately 60-70 percent of the local chord length from the leading edge; also observed and reported by Maines and Arndt [44].

The main contrast between the smooth and roughened cases can be noted close to the tip, especially in $\mathrm{x} / \mathrm{C}_{0}<0.1$, where the flow shows different separation lines and angles of interaction between the tip vortex and BL, Fig. 16. It is observed that while the smooth BL shows only one dominant separation line very close to the tip, the roughened case shows an extra separation line almost parallel to the tip vortex trajectory, Fig. 16b. For a rounded tip geometry, cross flow load distribution determines the location of the leading edge separation line and the strength of the circulation zone around it while the roughness separation line depends mostly on the turbulent friction introduced into the flow due to the interaction with the roughness elements.

To clarify how the turbulent friction introduced by the interaction of the BL and roughness elements affects the pressure field, the distributions of the pressure coefficient and turbulent viscosity ratio $\left(\mathrm{nu}_{t} / \mathrm{nu}\right)$ at the tip section, i.e. $\mathrm{z} / \mathrm{C}_{0}=0$, are presented in Fig. 17. As can be seen, roughness causes a huge increase in the turbulent viscosity ratio, as high as $\mathrm{nu}_{t} / \mathrm{nu}=27$, and consequently a large turbulent friction over the roughened area. This friction decelerates the BL close to the foil and forces an extra separation line, noted by the area having the lowest turbulent viscosity ratio. Before this separation line, the BL experiences an adverse pressure gradient which results in the tip vortex formation earlier than the separation line. For the smooth foil, the turbulent viscosity ratio is much lower and the BL only experiences one separation line very close to the tip. Consequently, 


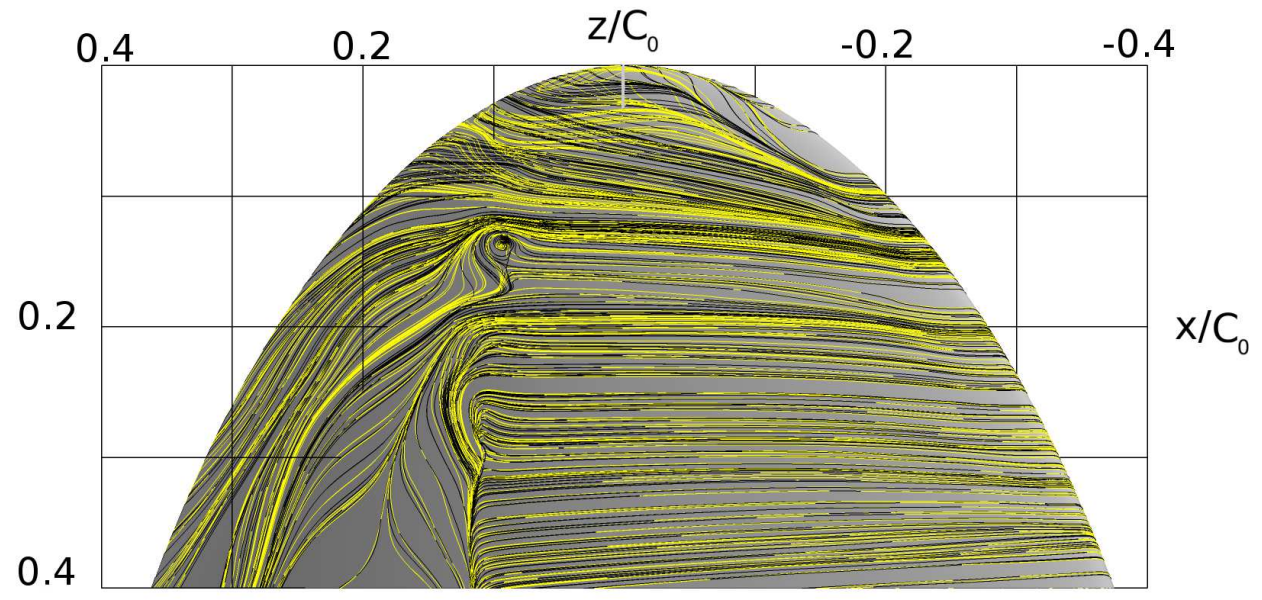

a

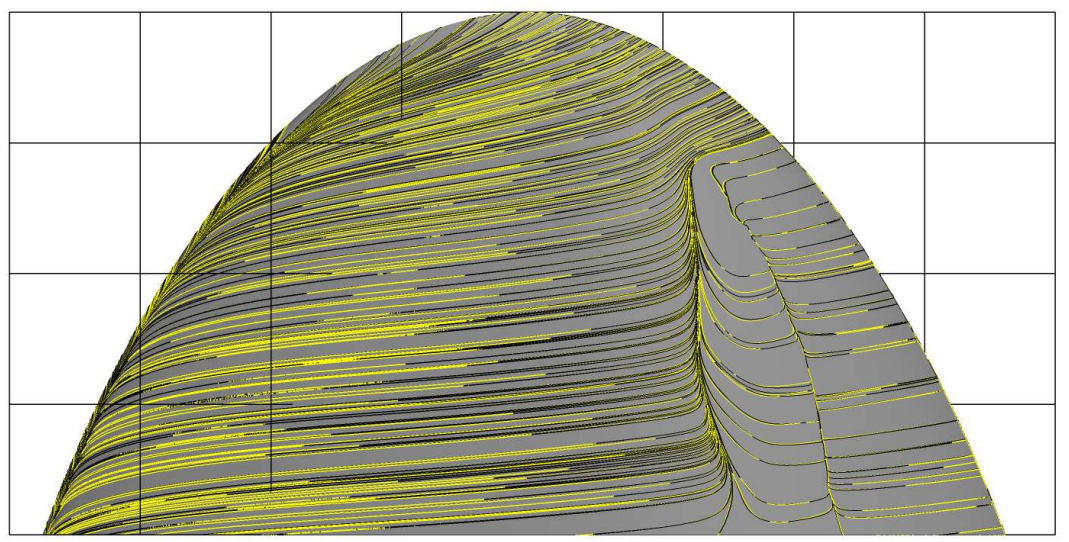

b

Fig. 15: Flow streamlines on the suction and pressure sides of the smooth foil presented by black lines and the roughness pattern (IV) presented by yellow lines. (a) Suction side view, (b) Pressure side view.

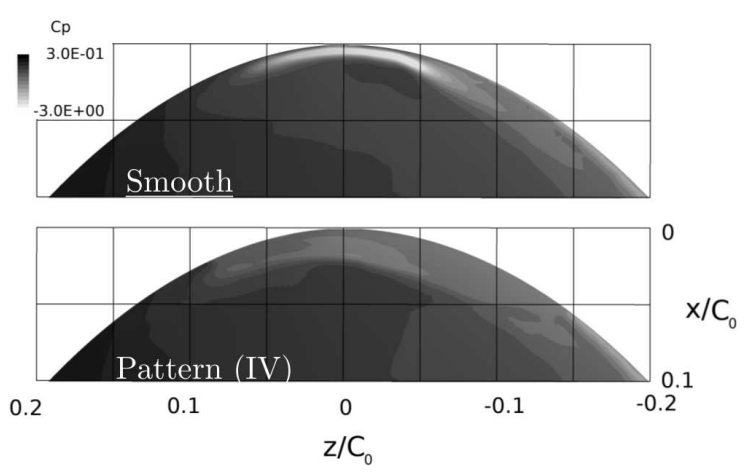

a

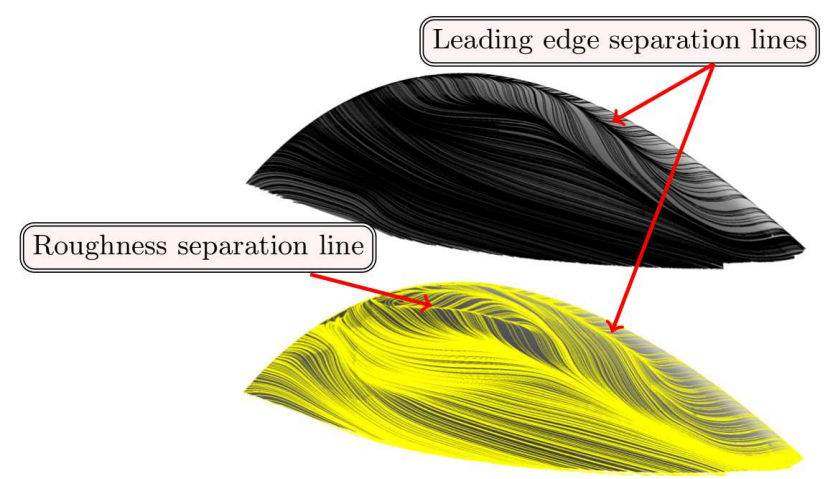

$\mathrm{b}$

Fig. 16: Pressure coefficient and flow streamlines around the tip of the foil. (a) Pressure coefficient, (b) Streamlines.

in the smooth foil the lowest pressure region which represents the tip vortex core happens close to the tip. The comparison of pressure coefficients of Fig. 17 shows that the tip vortex of the smooth foil is stronger and 


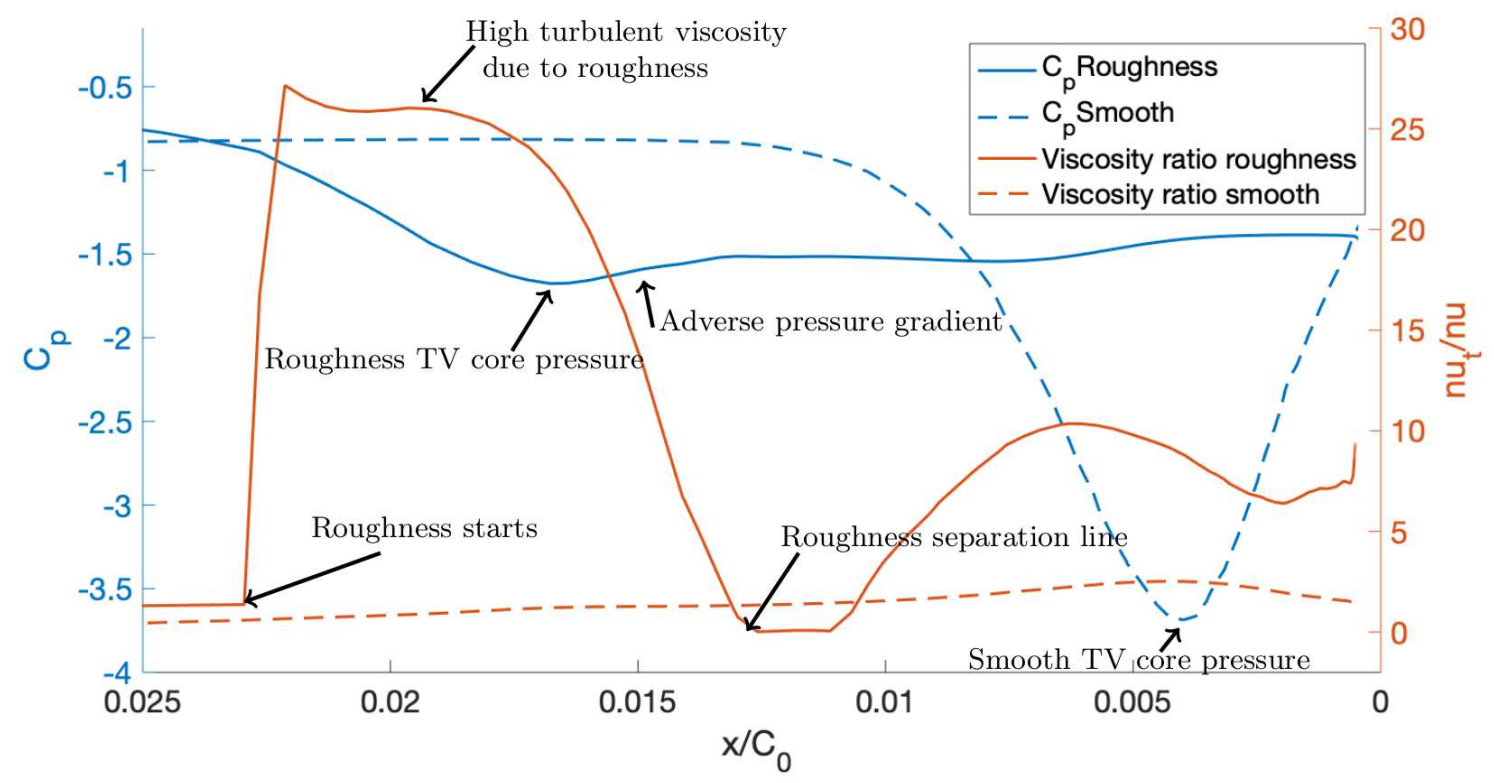

Fig. 17: Distribution of the averaged pressure coefficient and turbulent viscosity ratio $\left(\mathrm{nu}_{t} / \mathrm{nu}\right)$ at $\mathrm{z} / \mathrm{C}_{0}=0$ section of the suction side.

has lower core pressure value even in its initiation stage. This is interesting that the pressure value at the tip edge, i.e. $\mathrm{x} / \mathrm{C}_{0}=0$, and also before the roughness pattern, i.e. $\mathrm{x} / \mathrm{C}_{0}=0.025$, are very similar in the smooth and roughened cases. One can assume that roughness alters the formation of circulation zone before the leading edge separation line, and consequently changes the pressure distribution. In Fig. 18, a detailed investigation of the near tip region is presented to determine how roughness affects the relation between BL characteristics and vortex roll-up. The figure includes the flow streamlines close to the foil surface, and the vorticity distributions as well as the velocity contours at different cross sections for the smooth and roughened cases.

As the flow moves from leading edge cross sections towards the mid-chord cross section, i.e. from z $/ \mathrm{C}_{0}=-0.06$ to $\mathrm{z} / \mathrm{C}_{0}=0$, a dominant circulation zone forms behind the leading edge separation line. The size of this circulation zone depends on the distance between the leading edge and the region where the flow is concentrated on the inboard area. The area containing the concentrated flow is closer to the tip in the smooth condition, and therefore the circulation zone covers a smaller area compared to the roughened case which then results in a stronger rotational region and tip vortex strength already in its initial formation for the smooth condition. In the following cross sections, the roughness separation line prevents the flow towards the tip leading edge and pushes the concentrated flow streamlines inboard. This forms a circulation zone that covers the area between the tip separation line and the roughness separation line. As can be seen from the results of the section $\mathrm{z} / \mathrm{C}_{0}=0$, having circulation zone over a larger are results in lower vorticity distribution around the tip vortex in the roughened case compared to the smooth case. 


\subsection{Resolving flow around roughness elements}

When the flow around the roughness elements is resolved more flow structures are captured in the numerical simulations, Fig. 19. Note that the two roughness cases can be considered to represent different types of roughness, where the wall function simulation models an isotropic sand grain roughness while the roughness resolved mimics a more sparse distribution of roughness elements.

Compared to the smooth condition, in the roughness wall modelling approach the vortical structure formed on the leading edge and trailing area are increased. The leading edge structure is obviously larger than the one observed in the smooth condition leading to a much larger vortex tube in the leading edge region. The area ttablehat contains the trailing vortices, i.e. $(0.05,0.05)<\left(\mathrm{x} / \mathrm{C}_{0}, \mathrm{z} / \mathrm{C}_{0}\right)<(0.1,0.2)$, has also become larger with more and finer structures forming and separating compared to the smooth condition. The roughness resolved case clearly differs on the foil with small structures generated from the roughness elements in the whole region on the foil tip.

Another obvious difference between the flow structures of these three cases is the level of small structures surrounding the tip vortex. While both the smooth and wall modelled results show interactions of very fine small structures around the main tip vortex, the results of the resolved case shows a very much less structures at that part. If it is believed these small structures around the main tip vortex are the products of the roll-up process, then the lower structures around the tip vortex in the roughness resolved flow condition indicates that in this condition the roll-up process is the weakest. This, however, could not be captured by the wall modelling approach.

The distribution of the vorticity magnitude at the section of $\mathrm{z} / \mathrm{C}_{0}=0$ and 0.1 are presented in Fig. 20 and Fig. 21. While in the wall modelled case there is a distinct tip vortex formed from the roll-up of the pressure side flow, in the resolved condition the presence of roughness elements interacts with the roll-up and prevents the formation of a strong tip vortex. This can be observed in both of the sections where the vorticity magnitude distributions are presented.

In Fig. 22a (Multimedia view) and Fig. 22b (Multimedia view), comparison of a snapshot of experimental observations and numerical results of resolving flow around roughness elements are presented. In the numerical results, the iso-surface of Q-criterion is used to represent vortical structures and an iso-surface of pressure is employed to highlight TVC. It should be noted that the concentration of roughness elements used in the experimental test is much lower than the one used in the numerical simulation. Even though, the comparison clearly shows a general good agreement between numerical results and experimental observations in terms of the extent of TVC and its development.

The tip vortex cavitation inception results for having roughness on the leading edge, tip and trailing edge regions of the suction side, Case (IV), are presented in Fig. 23. In this figure, the experimental measurements related to the uniform roughness sand distribution and the randomly sparse distribution of roughness sand grains are included. The uniform distribution can be correlated to the numerical wall modelling approach where a uniform distribution of sand grains is assumed, and the sparse distribution can be correlated to the randomly distributed roughness elements and resolving approach. As discussed before, the predicted tip vortex strength is lower for the resolved flow condition resulted from having a weaker roll-up and also a more scattered vorticity distribution compared to the wall modelled results. Both of the experimental measurements and numerical results predict a weaker tip vortex in the randomly distributed roughness elements condition. As discussed before, this can 
be related to having a weaker roll-up and also a more scattered vorticity distribution in this surface roughness condition compared to the uniform roughness distribution and its related wall modelled results. It can be noted that the general agreement between different roughness modelling approaches and their related experimental tests is satisfactory.

\subsection{Tip vortex velocity profiles}

Numerical and experimental distributions of the vortex velocity for the smooth and case (IV) conditions are presented in Fig. 24. By assuming that the vortex core center have zero rotational velocity, half of the distance between maximum and minimum tangential velocities is considered as the vortex radius which then is used in these figures for normalizations. In each condition, the velocity is normalized using the inlet velocity.

The tangential velocity comparison shows a very good agreement between numerical results and experimental measurements in the smooth and roughened conditions. Both the magnitude of the tangential velocity and its variation over vortex core are predicted reasonably good. This corresponds to an accurate prediction of angular momentum distributions in the simulations. The results indicate that in the roughened surface condition the magnitude of the tangential velocity is decreased compared to the smooth condition. The vortex core axial velocity, i.e. streamwise velocity, shows more discrepancy between numerical results and experimental measurements. While in the numerical results the maximum axial velocity is predicted to happen relatively close to the vortex core center, the experimental measurements show the axial velocity peak happens slightly off the center, e.g. in the smooth condition it happens at $\mathrm{r} / \mathrm{r}_{v}=0.5$. This discrepancy can be the result of the all possible uncertainties available between CFD and experimental methods, e.g. roughness size and its distribution over the surface.

\section{Conclusion}

In this paper, the possibility of artificial roughness application to suppress tip vortex and consequently mitigate cavitation inception is demonstrated by using numerical and experimental analyses of the flow over an elliptical foil. The analysis includes the optimization of the roughness pattern and its size based on the balance of the tip vortex cavitation suppression and the performance degradation.

Both the numerical results and experimental measurements prove the capability of using roughness to suppress the tip vortex cavitation inception. The analysis shows a decrease in the tip vortex cavitation inception as large as $33 \%$ in the optimized roughness pattern compared to the smooth foil condition where the drag force increase is observed to be less than $2 \%$.

The roughness area optimization is conducted by considering two flow criteria to find effective regions in the formation and development of tip vortex flows, the vortical structures distribution and the flow streamlines passing through the tip vortex region. Based on the analyses of these two criteria over the smooth foil condition, different roughness area configurations are created. The comparison between the cavitation inception and the foil performance of these configurations shows that application of roughness on the leading edge, tip and trailing edge of the suction side is the optimum roughness configuration. The impact of the roughness height on the tip vortex is noted, and it is observed that for the studied condition, the roughness size of $250 \mu \mathrm{m}$ is sufficient. The detailed investigation of the near tip region characteristics of the boundary layer and vortex roll-up in 
the smooth and roughened cases shows the impact of the separation lines on the vortex development. It is highlighted that while the smooth foil only has one dominant separation line close to the tip, the roughened foil experiences an extra separation lines due to the roughness presence. This extra separation line is believed to be responsible for prevention of vorticity accumulation close to the tip and consequently leading to a weaker tip vortex compared to the smooth foil condition.

Further, both an isotropic sand grain roughness and a sparse distribution of roughness elements are considered in both the simulations and the experiments. The difference between the cavitation inception predicted by the wall modelled and resolved flow is found to be considerable, where the predicted cavitation inception by wall modelled approach is found to be close to the experimental measurements using sand grain roughness while the resolved case is similar to the experimental case with distributed roughness.

As the current study focuses only on one operating condition where the tip vortex is formed on the suction side of the foil, further studies on a wider range of operating conditions are necessary to obtain a generalized optimum roughness configuration, e.g. roughness pattern and size. However, the presented analysis is proved to be able to distinguish effective regions in tip vortex formation, and showed the possibility of tip vortex mitigation by using roughness with a small impact on the performance degradation. 


\section{Acknowledgements}

Financial support for this work has been provided by VINNOVA through the RoughProp project, Grant number 2018-04085. The simulations were performed on resources at Chalmers Centre for Computational Science and Engineering (C3SE) provided by the Swedish National Infrastructure for Computing (SNIC).

Data availability Raw data were generated at the cavitation tunnel facility of Kongsberg Maritime AB, Kristinehamn, Sweden. Derived data supporting the findings of this study are available from the corresponding author upon reasonable request.

\section{References}

[1] J. A. Hildebrand. Impacts of anthropogenic sound. In Marine mammal research: conservation beyond Crisis. JHU Press, 2005.

[2] W.J. Richardson, C.R. Greene, C.I. Malme, and D.H.. Thomson. Marine mammals and noise. Academic Press, San Diego, Calif, 1995.

[3] B. Aktas, S. Turkmen, W. Shi, R. Sampson, P. Fitzsimmons, E. Korkut, and M. Atlar. Underwater radiated noise investigations of cavitating propellers using medium size cavitation tunnel tests and full-scale trials. In Proceedings of Fourth International Symposium on Marine Propulsors,SMP'15, Austin, Texas, USA, 2015.

[4] C. E. Brennen. Cavitation and Bubble Dynamics. Oxford University Press, New York, 1995.

[5] Bernd Budich, S. J. Schmidt, and N. A. Adams. Numerical simulation and analysis of condensation shocks in cavitating flow. Journal of Fluid Mechanics, 838:759-813, 2018.

[6] J. Bosschers. Propeller tip-vortex cavitation and its broadband noise. PhD thesis, University of Twente, 2018.

[7] J.-K. Choi and G.L. Chahine. Noise due to extreme bubble deformation near inception of tip vortex cavitation. Physics of Fluids, 16(7):2411-2418, 2004.

[8] A. Asnaghi, U. Svennberg, and R.E. Bensow. Numerical and experimental analysis of cavitation inception behaviour for high-skewed low-noise propellers. Applied Ocean Research, 79:197 - 214, 2018.

[9] G.L. Chahine, G.F. Frederick, and R.D. Bateman. Propeller tip vortex cavitation suppression using selective polymer injection. Journal of Fluids Engineering, Transactions of the ASME, 115(3):497-503, 1993.

[10] D.H. Fruman and S.S. Aflalo. Tip vortex cavitation inhibition by drag-reducing polymer solutions. Journal of Fluids Engineering, Transactions of the ASME, 111(2):211-216, 1989.

[11] A. Rivetti, M. Angulo, C. Lucino, and S. Liscia. Mitigation of tip vortex cavitation by means of air injection on a Kaplan turbine scale model. In IOP Conference Series: Earth and Environmental Science, volume 22, 2014. 
[12] S.I. Park, S.J. Lee, G.S. You, and J.C. Suh. An experimental study on tip vortex cavitation suppression in a marine propeller. Journal of Ship Research, 58(2):1-11, 2014.

[13] A. Amini, J. Seo, S.H. Rhee, and M. Farhat. Mitigating tip vortex cavitation by a flexible trailing thread. Physics of Fluids, 31(12), 2019.

[14] J. Katz and J. Galdo. Effect of roughness on rollup of tip vortices on a rectangular hydrofoil. J. Aircraft, 26:247-253, 1989.

[15] C. A. Johnsson and O. Ruttgerson. Leading edge roughness: a way to improve propeller tip vortex cavitation. In Propeller Shafting Symposium, 1991.

[16] O. Philipp and P. Ninnemann. Wirkung von fluegelrauigkeiten auf kavitation und erregung. In Maritime Speech Day, Technical University Hamburg Harburg, 2007.

[17] S. C. C. BAILEY and S. TAVOULARIS. Measurements of the velocity field of a wing-tip vortex, wandering in grid turbulence. Journal of Fluid Mechanics, 601:281-315, 2008.

[18] H.C. Ghimire and S.C.C. Bailey. An experimental investigation of wing-tip vortex decay in turbulence. Physics of Fluids, 29(3), 2017.

[19] C. Kruger, N. Kornev, and L. Greitsch. Influence of propeller tip roughness on tip vortex strength and propeller performance. Ship Technology Research, 63(2):110-120, 2016.

[20] W. G. Souders and G. P. Platzer. Tip vortex cavitation characteristics and delay of inception on a threedimensional hydrofoil. Naval Ship Research and Development Center,, 1981.

[21] R.E.A. Arndt and A.P. Keller. Water quality effects on cavitation inception in a trailing vortex. Journal of Fluids Engineering, 114(3):430 - 438, 1992.

[22] B. Zhang, J. Lou, C.-W. Kang, A. Wilson, J. Lundberg, U. Svennberg, and R.E. Bensow. CFD modeling of propeller tip vortex over large distances. International Journal of Offshore and Polar Engineering, 24(3):181-183, 2014.

[23] A. Ducoin, J.A. Astolfi, and J.F. Sigrist. An experimental analysis of fluid structure interaction on a flexible hydrofoil in various flow regimes including cavitating flow. European Journal of Mechanics, B/Fluids, $36: 63-74,2012$.

[24] E. Amromin. Analysis of cavitation inception and desinence behind surface irregularities. Physics of Fluids, $28(7), 2016$.

[25] R.E.A. Arndt, V.H. Arakeri, and H. Higuch. Some observations of tip-vortex cavitation. Journal of Fluids Mechanics, 229(1):269 - 289, 1991.

[26] P.C. Pennings, J. Westerweel, and T.J.C. van Terwisga. Flow field measurement around vortex cavitation. Experiments in Fluids, 56(11):206 - 218, 2015.

[27] P.C. Pennings. Dynamics of vortex cavitation. PhD -thesis, TU Delft University, 2016. 
[28] A. Asnaghi. Computational modelling for cavitation and tip vortex flows. PhD -thesis, Chalmers University of Technology, 2018.

[29] H. Liu, X. Qin, S. Ahmad, Q. Tong, and J. Zhao. Molecular dynamics study about the effects of random surface roughness on nanoscale boiling process. International Journal of Heat and Mass Transfer, $145: 118799,2019$.

[30] J.W.R. Peeters and N.D. Sandham. Turbulent heat transfer in channels with irregular roughness. International Journal of Heat and Mass Transfer, 138:454 - 467, 2019.

[31] C. Fureby. ILES and LES of complex engineering turbulent flows. In European Conference on Computational Fluid Dynamics, ECCOMAS CFD, 2006.

[32] R.E. Bensow and G. Bark. Implicit LES predictions of the cavitating flow on a propeller. Journal of Fluids Engineering, 132(4):041302, 2010.

[33] A. Asnaghi. Developing computational methods for detailed assessment of cavitation on marine propellers. Licentiate thesis, Chalmers University of Technology, 2015.

[34] J.C.R. Hunt, A.A. Wray, and P. Moin. Eddies, stream, and convergence zones in turbulent flows. Center for Turbulence Research Report CTR-S88, 1998.

[35] V. Kolar. Vortex identification: New requirements and limitations. International Journal of Heat and Fluid, 28, 2007.

[36] J.J.A. Schot, P.C. Pennings, M.J.B.M. Pourquie, and T.J.C. van Terwisga. Modelling of tip vortex cavitation for engineering applications in OpenFOAM. In 11th World Congress on Computational Mechanics, July 20-25, 2014, Barcelona, Spain.

[37] L. Chen, L. Zhang, X. Peng, and X. Shao. Influence of water quality on the tip vortex cavitation inception. Physics of Fluids, 31(2), 2019.

[38] A. Asnaghi, A. Feymark, , and R. Bensow. Numerical analysis of tip vortex flow. In Proceedings of the 19th Numerical Towing Tank Symposium (NuTTS'16),Oléron, French, pages 11 - 15, 2016.

[39] A. Asnaghi, , R.E. Bensow, and U. Svennberg. Implicit large eddy simulation of tip vortex on an elliptical foil. In Proceedings of Fifth International Symposium on Marine Propulsion (SMP 2017), Espoo, Finland, pages WB2-1, 2017.

[40] OpenFoam: The Open Source CFD Toolbox openfoam® foundation. http://www.openfoam.com. Accessed: 2020-5-14.

[41] R.E. Bensow and M. Liefvendahl. Implicit and explicit subgrid modeling in LES applied to a marine propeller. In 38th AIAA Fluid Dynamics Conference and Exhibit, 2008.

[42] A. C. DeVoria and K. Mohseni. Vortex sheet roll-up revisited. Journal of Fluid Mechanics, 855:299-321, 2018 . 
[43] A. Asnaghi, , R. Bensow, and U. Svennberg. Comparative analysis of tip vortex flow using RANS and LES. In Proceedings of VII International Conference on Computational Methods in Marine Engineering (MARINE 2017), Nantes, French, 2017.

[44] B.H. Maines and R.E.A. Arndt. Tip vortex formation and cavitation. Journal of Fluids Engineering, Transactions of the ASME, 119(2):413-419, 1997.

[45] B.H. Maines and R.E.A. Arndt. Viscous effects on tip vortex cavitation. In Intl. Symp. Cavitation Inception, ASME,N.Y., 1993. 

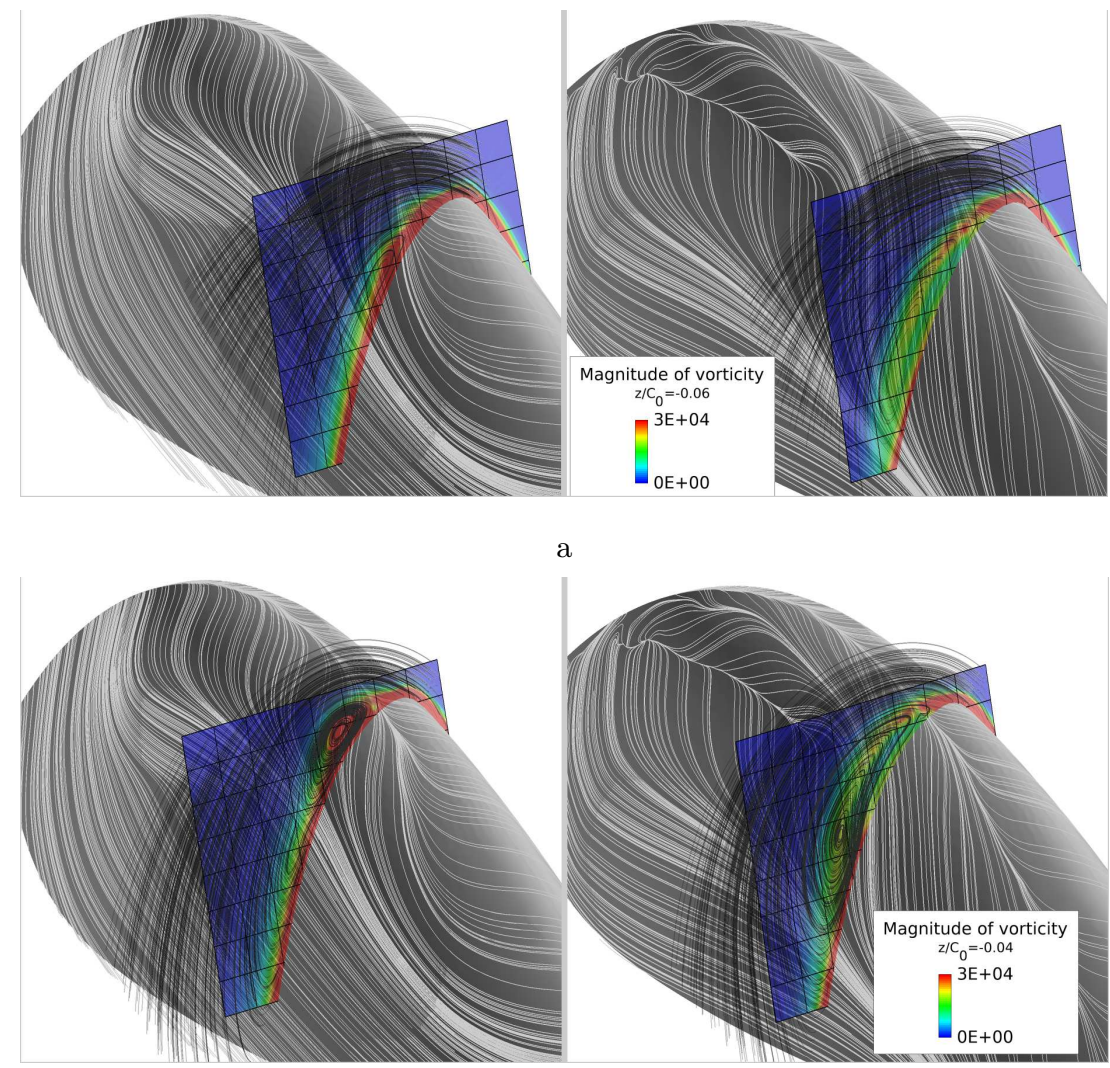

$\mathrm{b}$
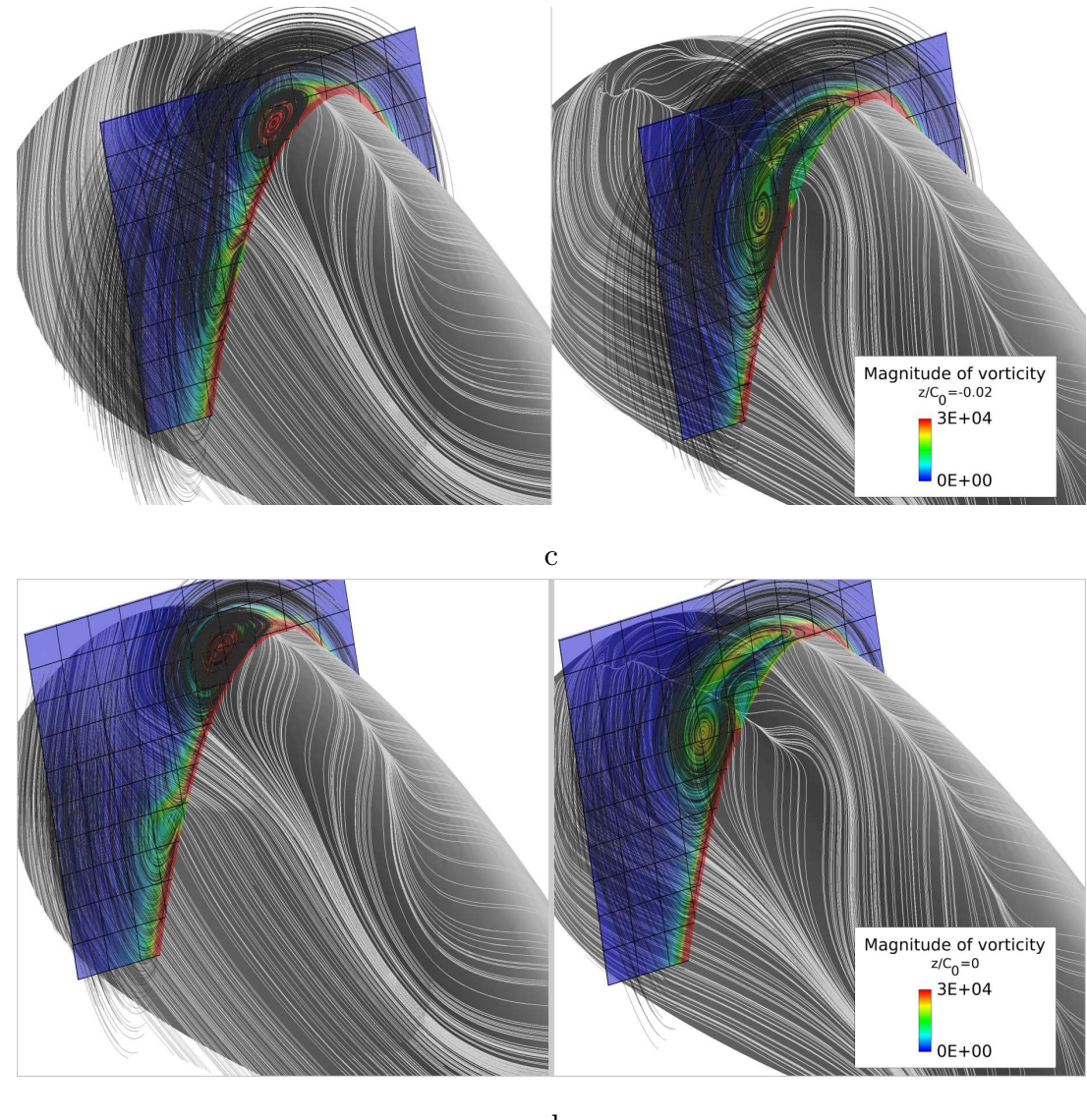

d

Fig. 18: Distribution of the averaged vorticity magnitude and velocity streamlines at different sections of the foil, isometric view of the suction side for the smooth and roughness pattern (IV). (a) z/ $\mathrm{C}_{0}=-0.06$, (b) $\mathrm{z} / \mathrm{C}_{0}=-0.04$, (c) $\mathrm{z} / \mathrm{C}_{0}=-0.02,(\mathrm{~d}) \mathrm{z} / \mathrm{C}_{0}=0$. 

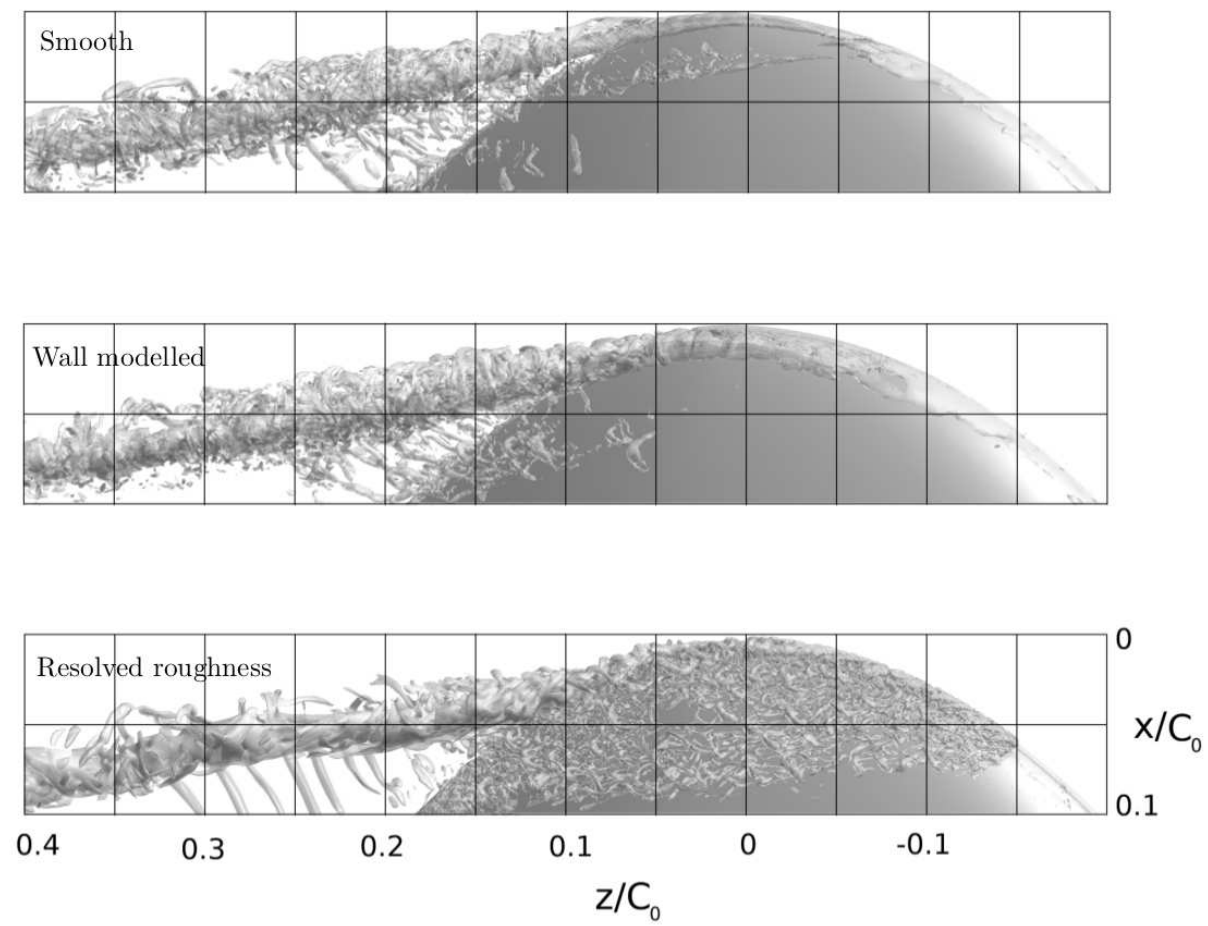

Fig. 19: Distribution of the iso-surface $\mathrm{Q}=800$ on the suction side.

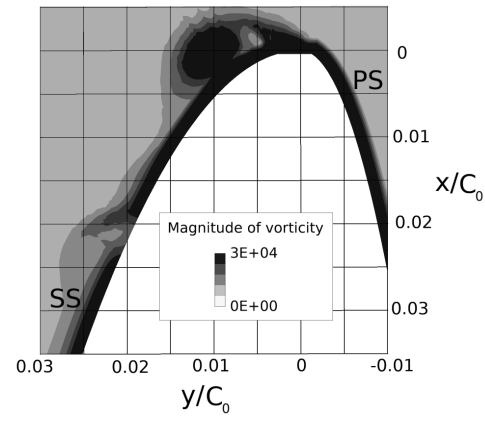

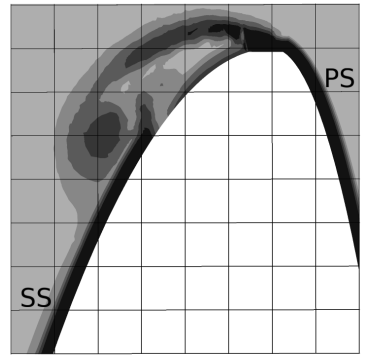

$\mathrm{b}$

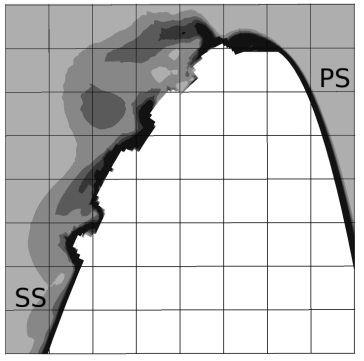

c

Fig. 20: Vorticity magnitude distribution at $\mathrm{z} / \mathrm{C}_{0}=0$, SS: suction side, PS: pressure side. (a) Smooth, (b) Case (IV), (c) Resolved roughness. 


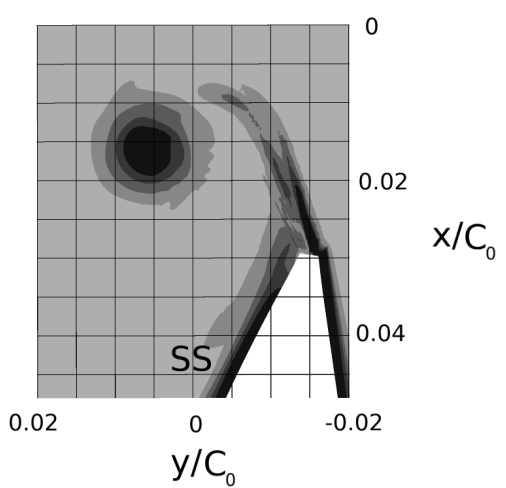

a
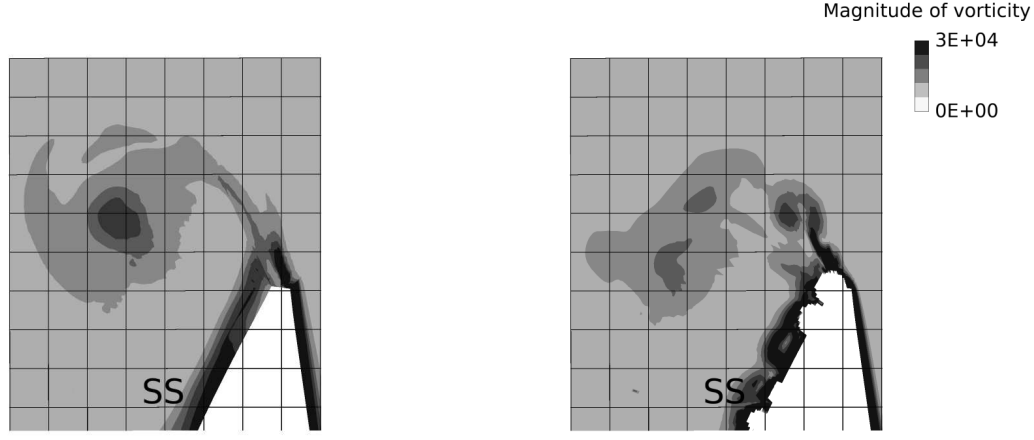

Fig. 21: Vorticity magnitude distribution at $\mathrm{z} / \mathrm{C}_{0}=0.1$, SS: suction side. (a) Smooth, (b) Case (IV), (c) Resolved roughness.

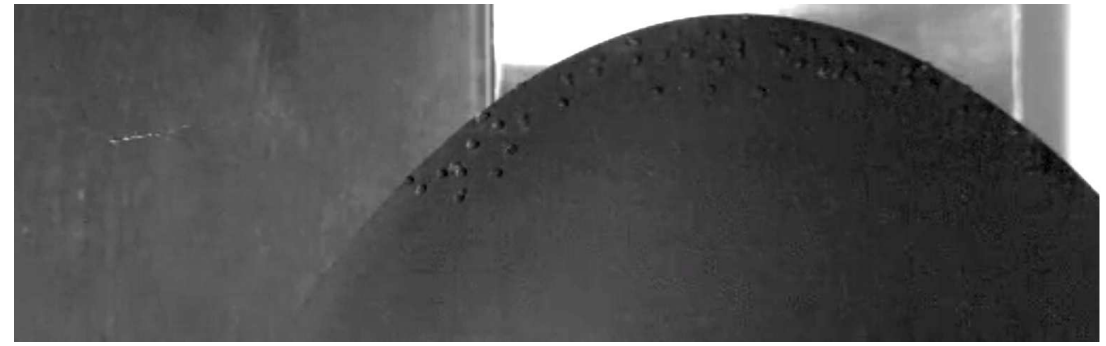

a

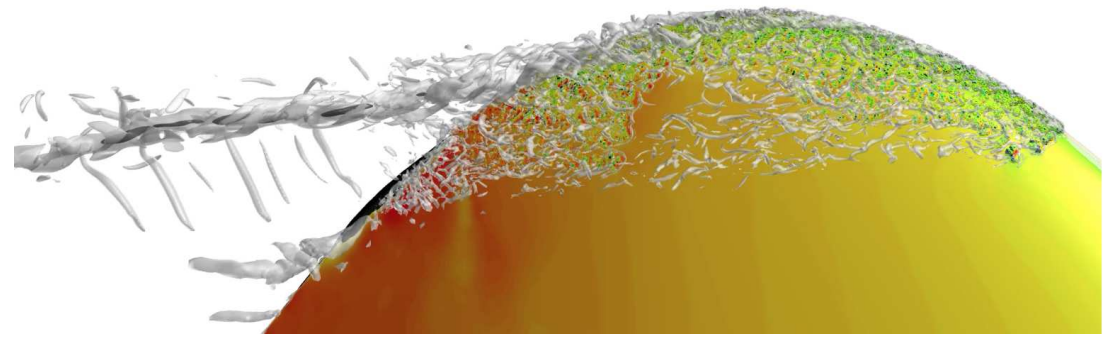

b

Fig. 22: Comparison of TVC between numerical results and experimental observations, tip zoomed view of the suction side. TVC is distinguished by colored ovals. (a) Experimental observations (Multimedia view), and (b) Numerical results (Multimedia view). 


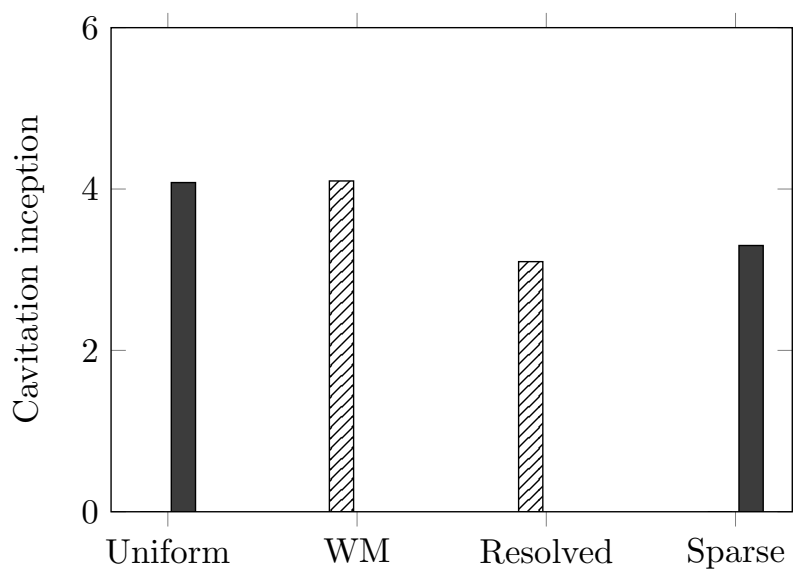

Fig. 23: Cavitation inception prediction of the roughness pattern (IV) for different numerical modelling approaches versus the experimental measurements with uniform and sparse sand grains distributions.

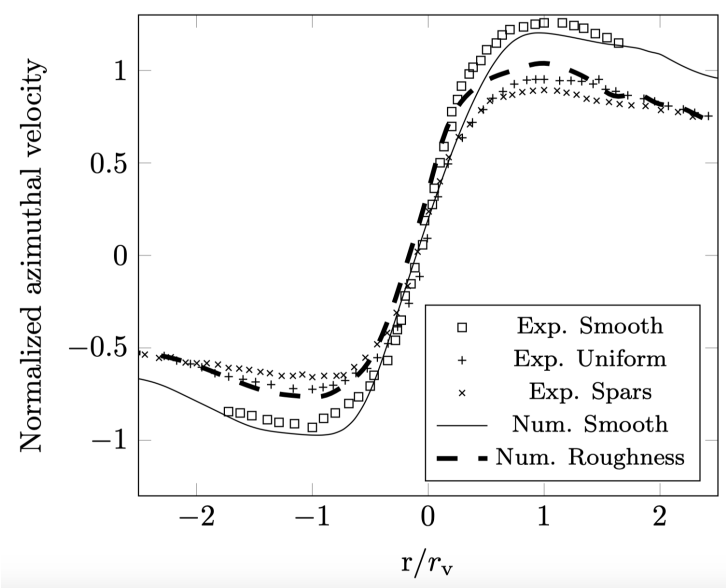

a

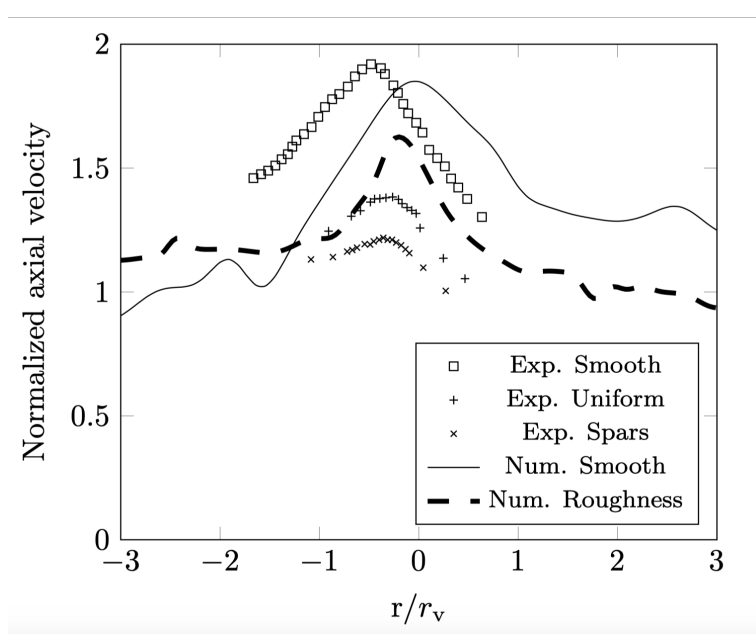

$\mathrm{b}$

Fig. 24: Variation of the normalized azimuthal and streamwise velocities in the vortex core region at $\mathrm{z} / \mathrm{C}_{0}=0.5$ downstream of the foil tip. (a) Normalized azimuthal velocity, and (b) Normalized streamwise velocity. 


\section{SS}

PS

(3)

(2)

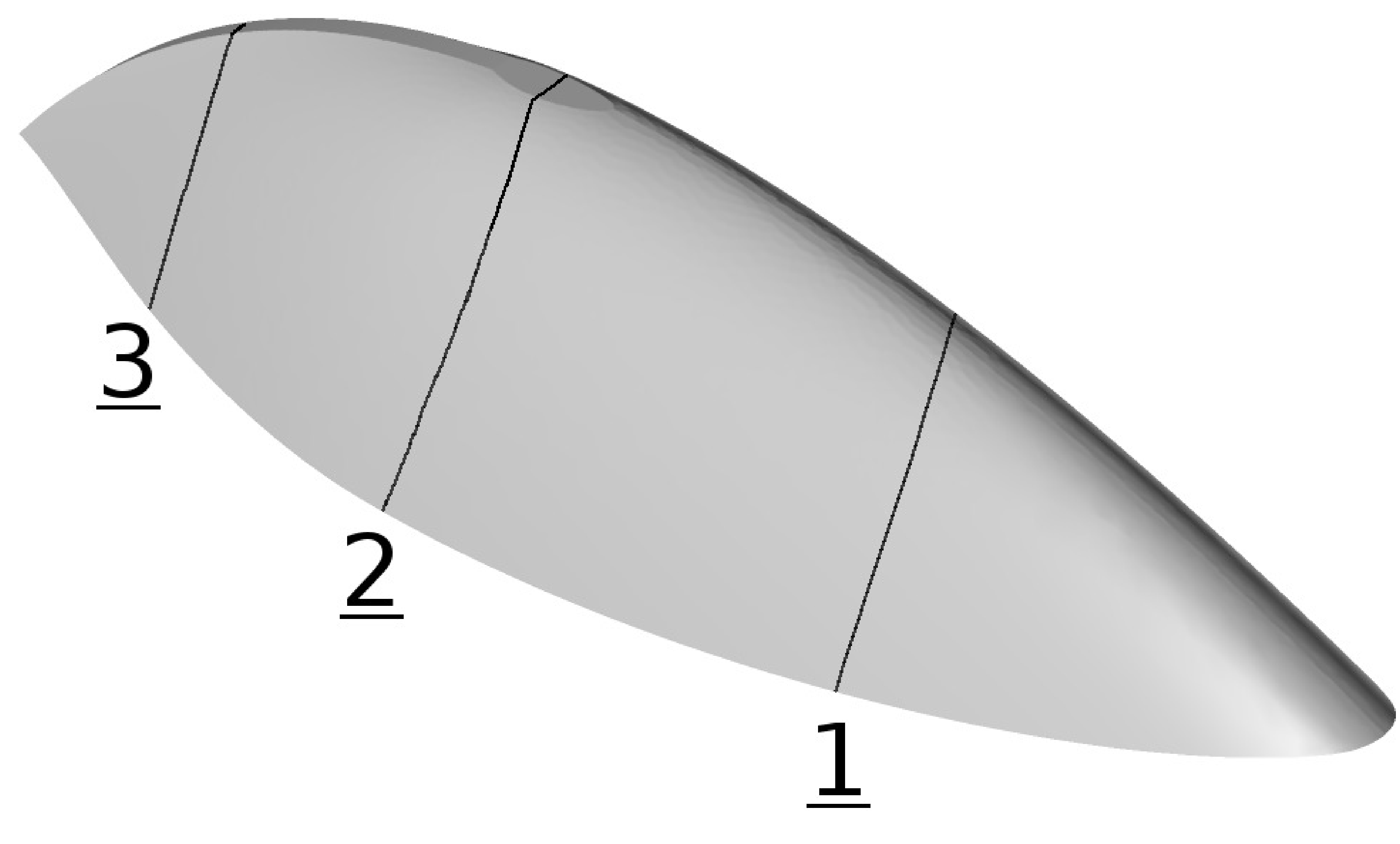

(1) 
Outlet

U: Zero gradient

Inlet

$\mathrm{U}=6.8 \mathrm{~m} / \mathrm{s}$

$\mathrm{P}$ : Zero gradient

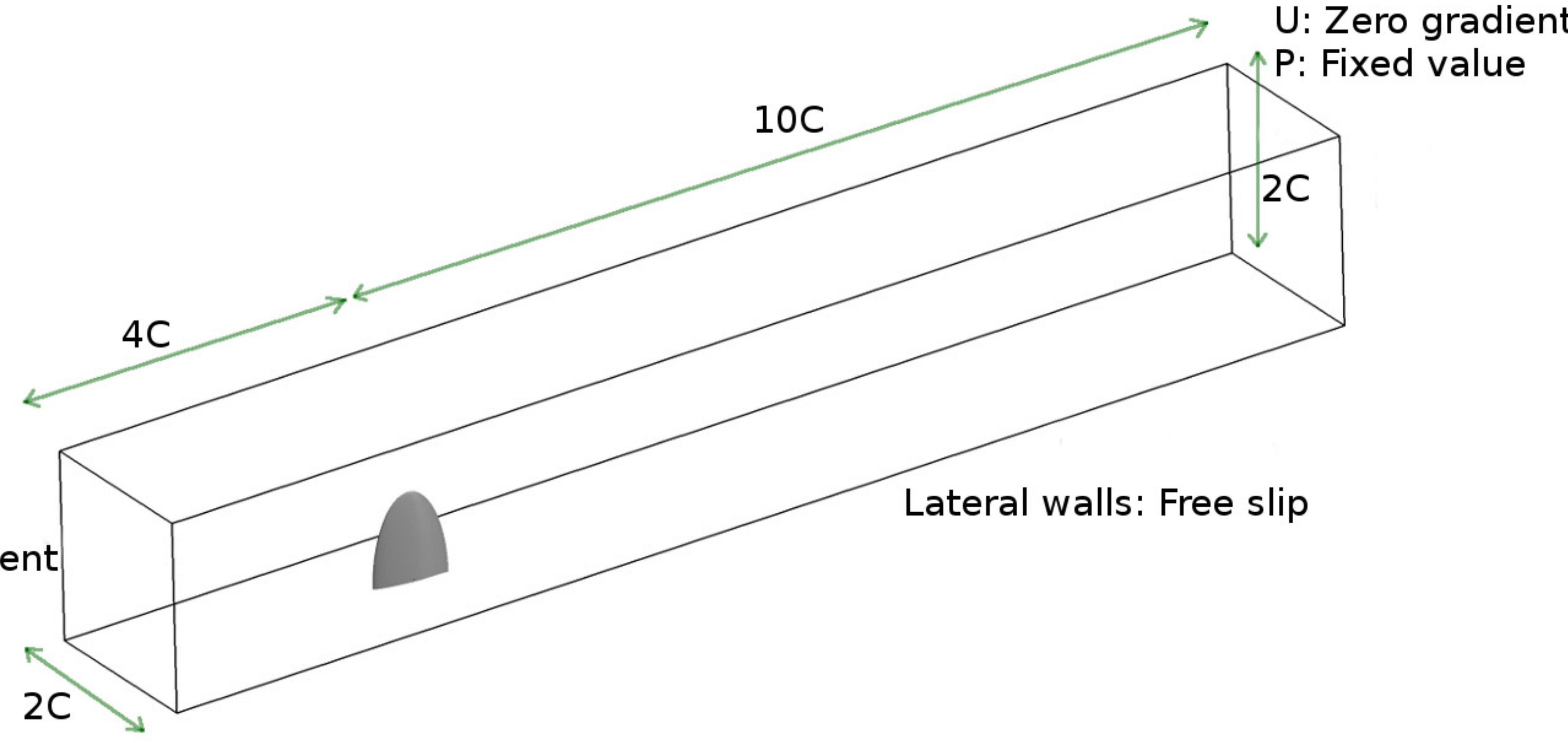


Tip region refinemnet

Tip vortex trajectory refinemnet

$0.07 \mathrm{C}_{0}$

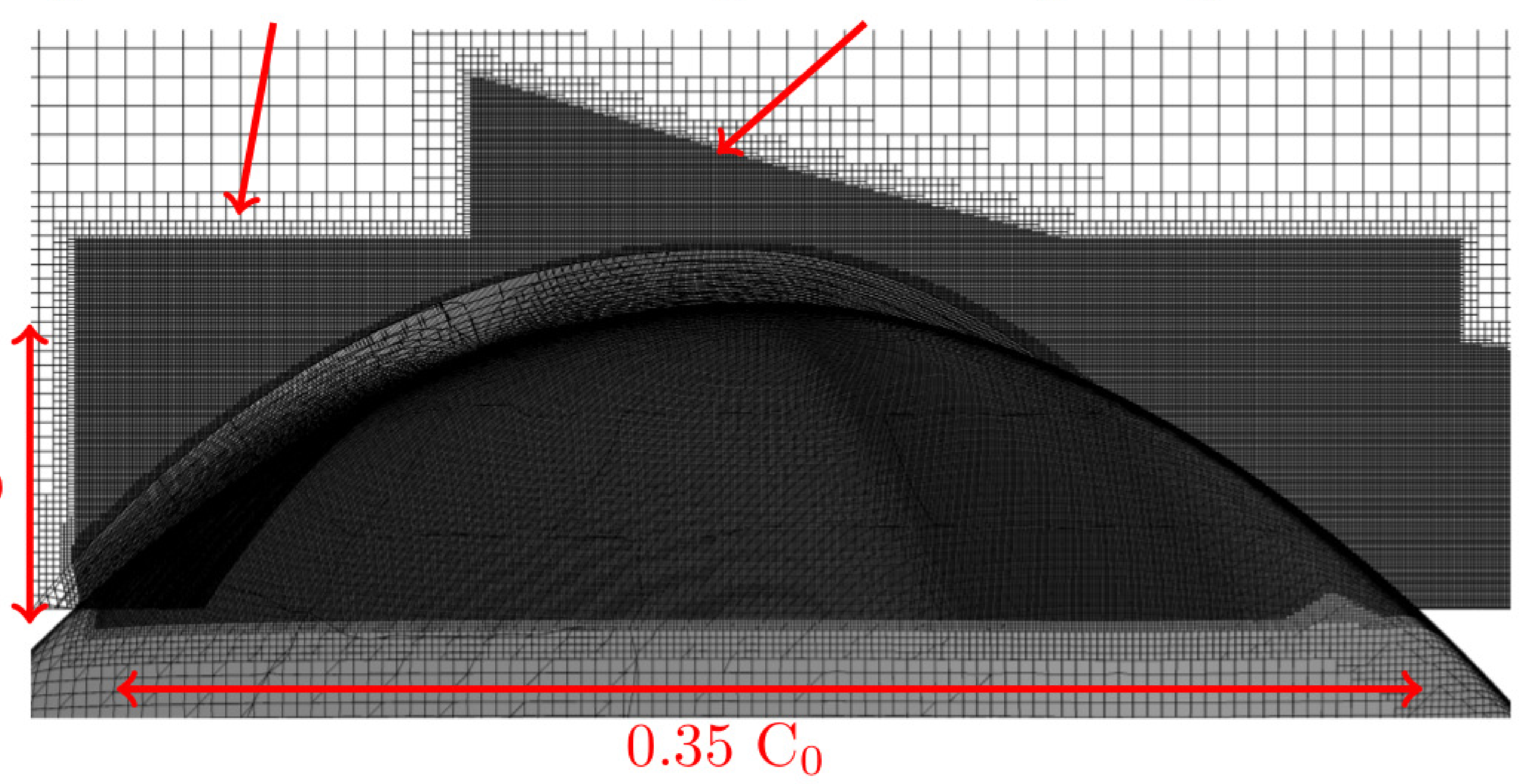


$+0$ \begin{tabular}{lll}
\hline &
\end{tabular}

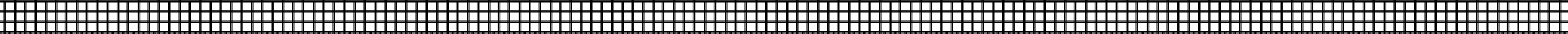

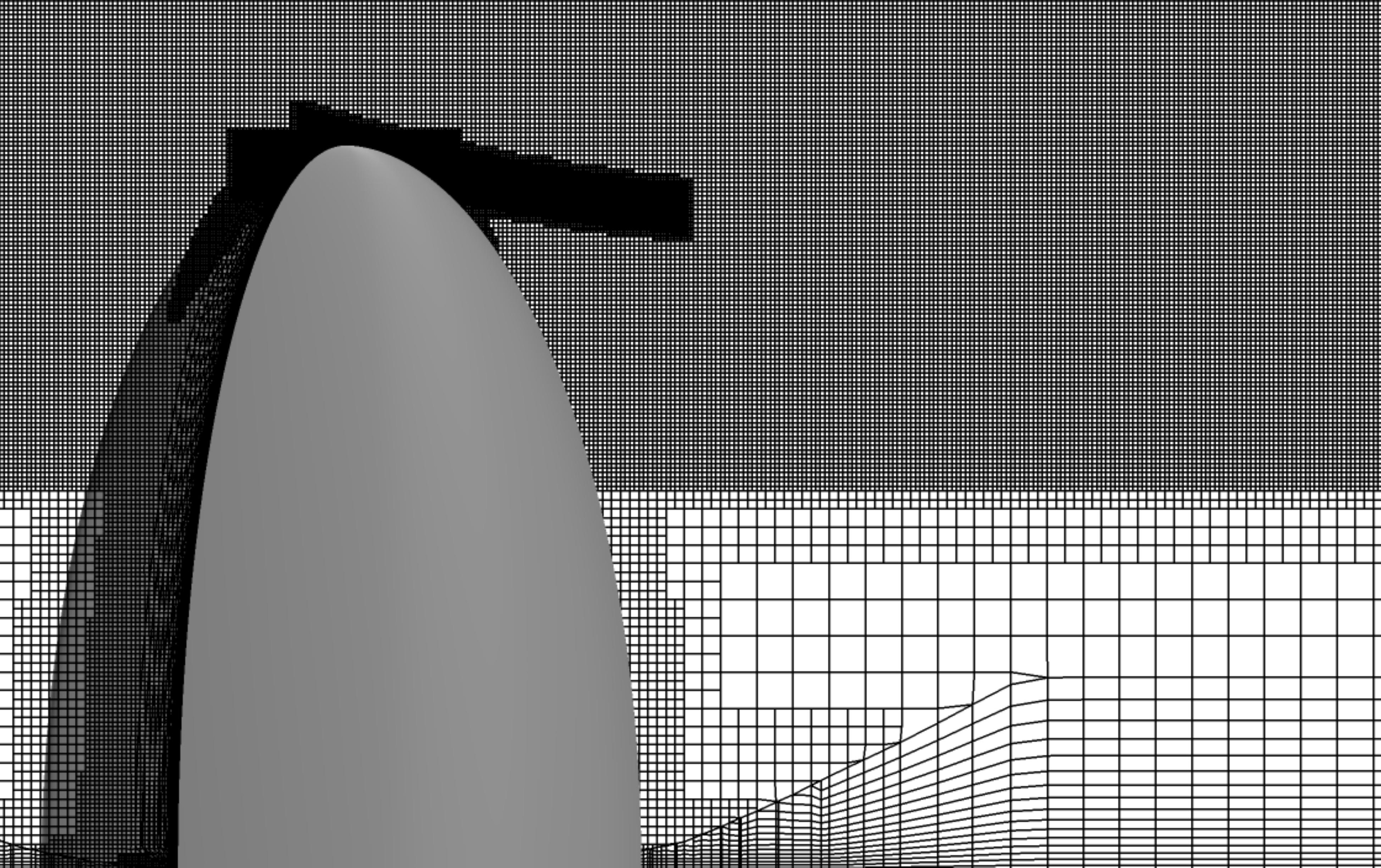





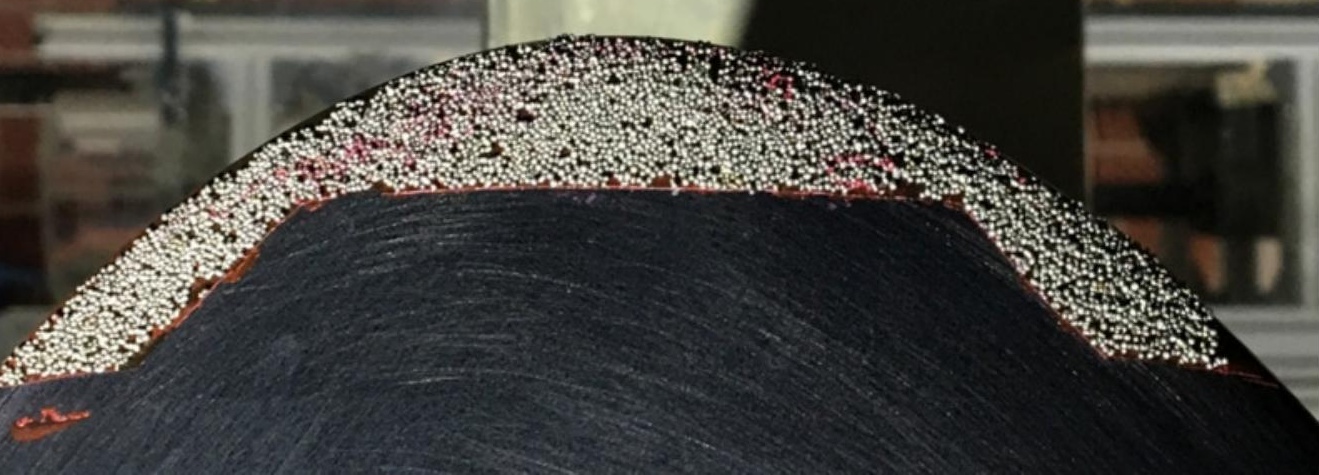





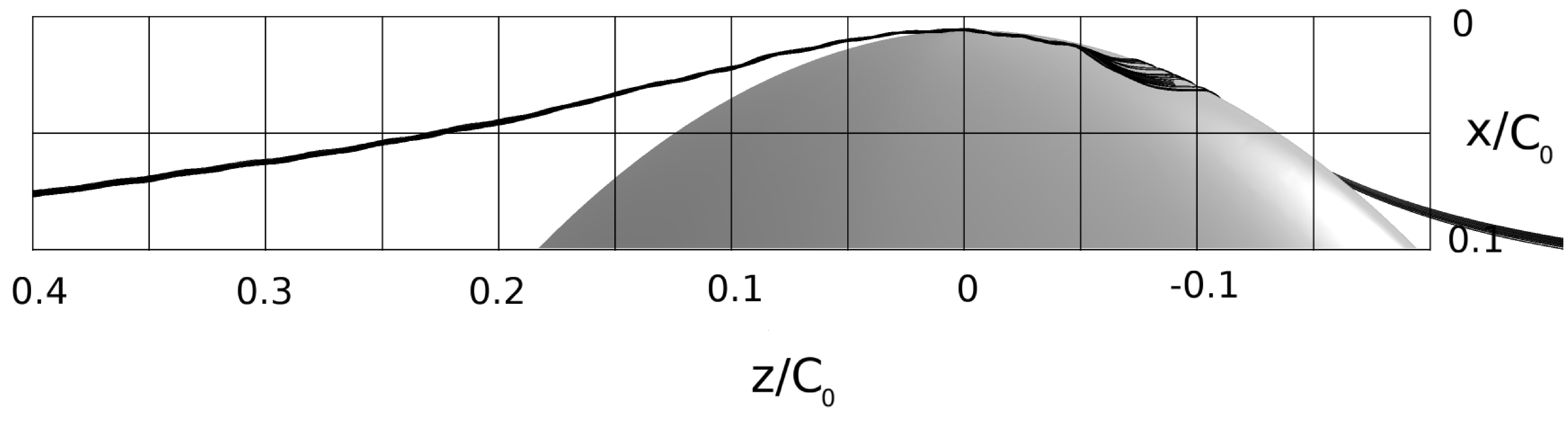




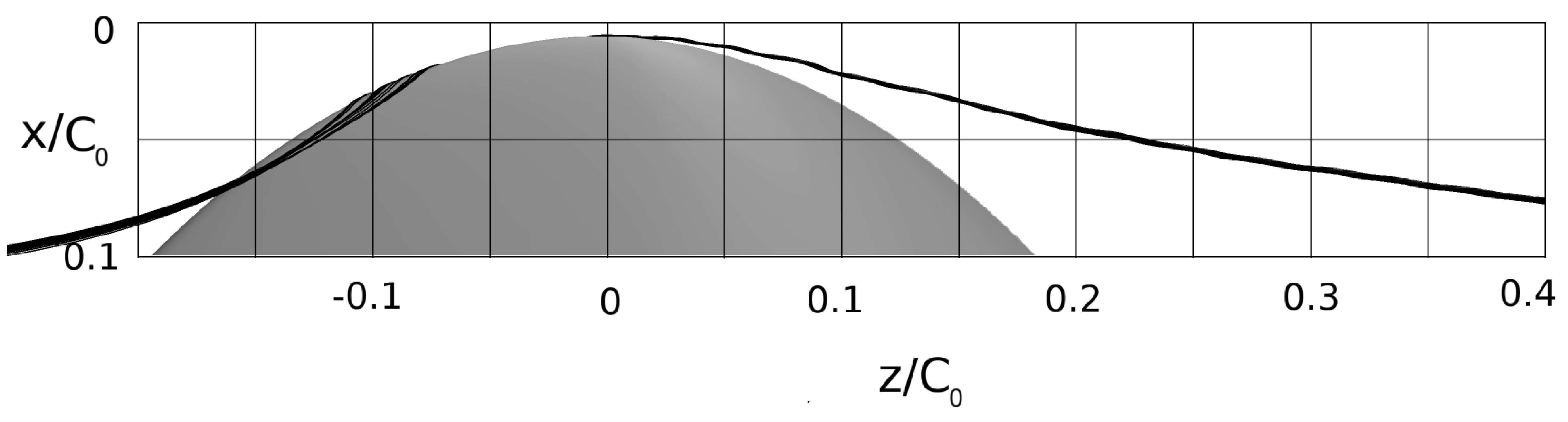




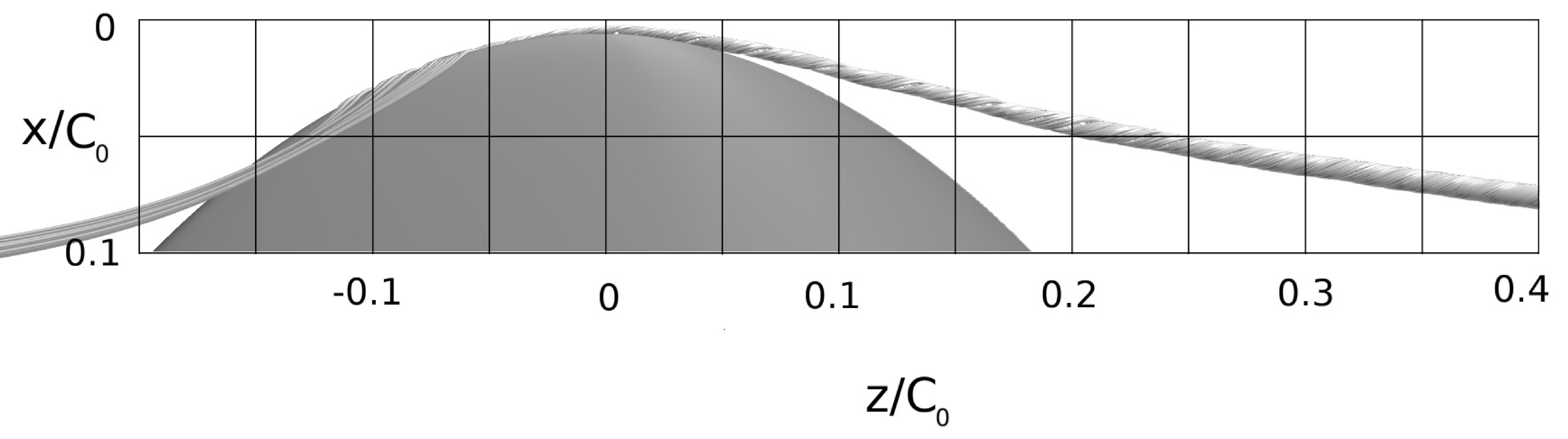



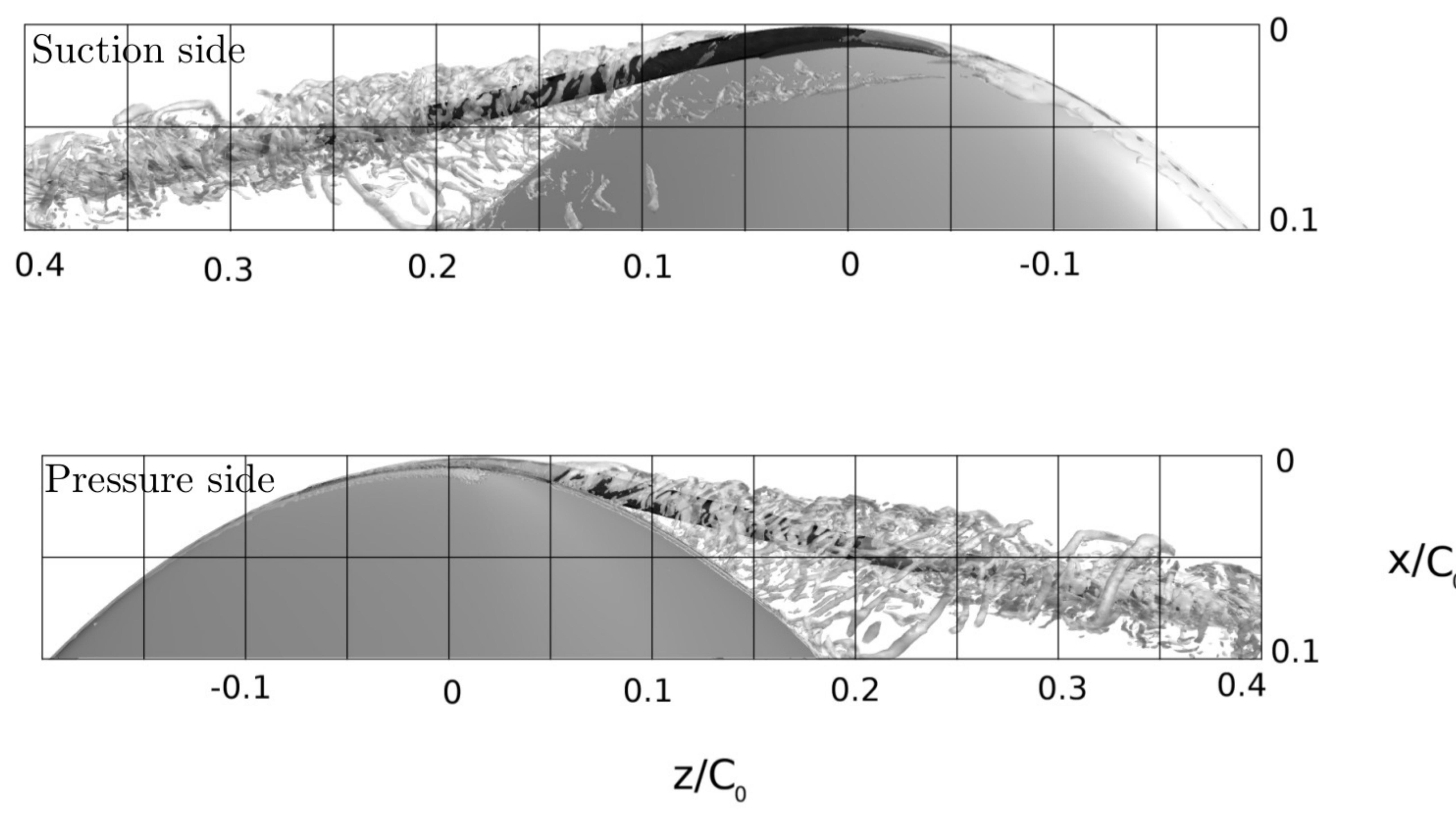


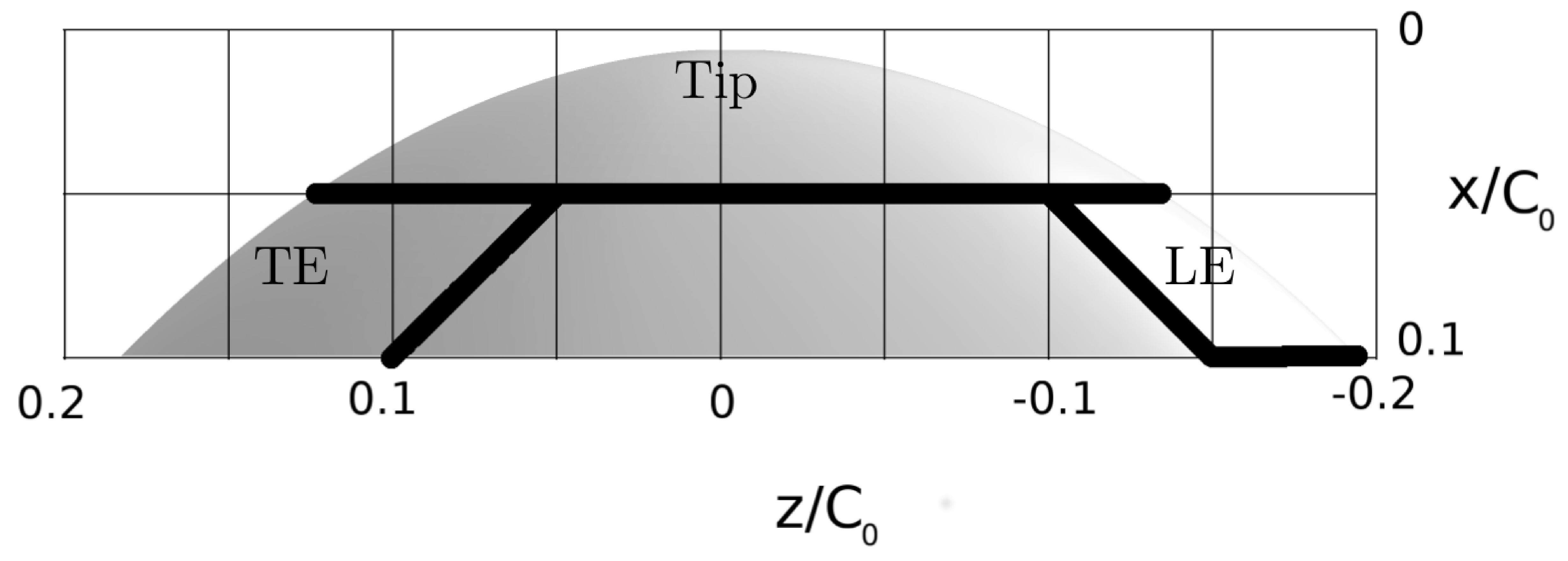




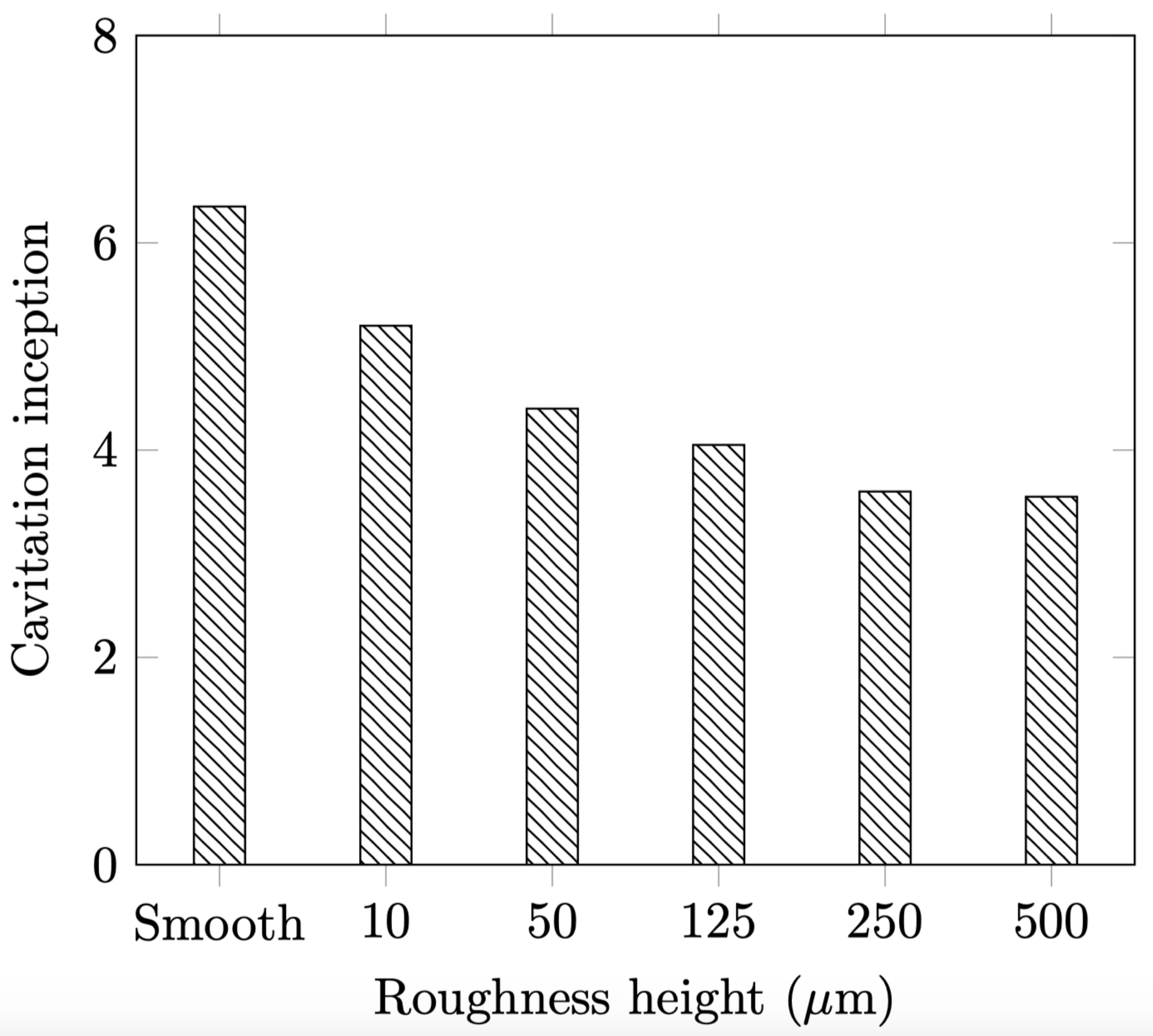




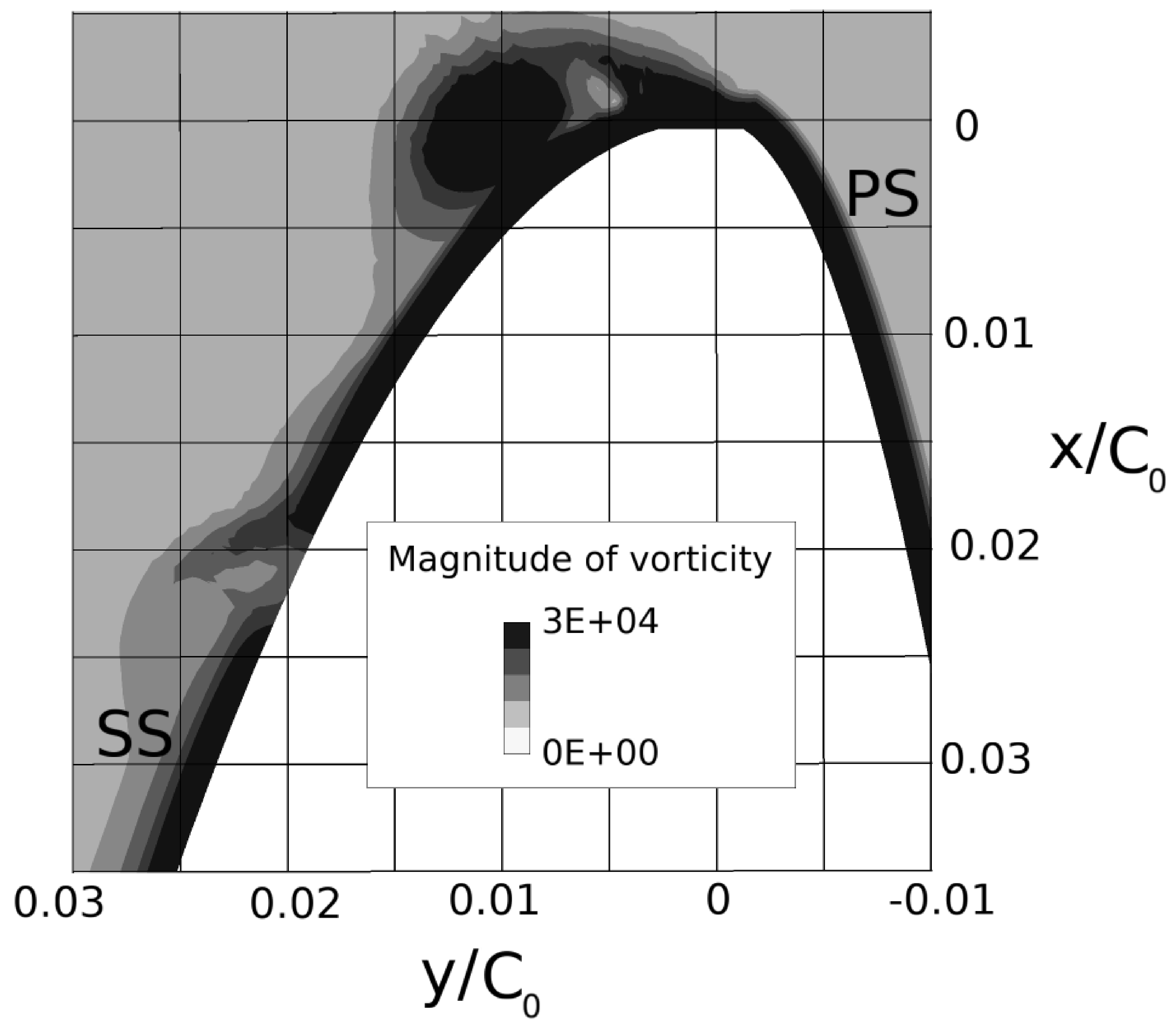




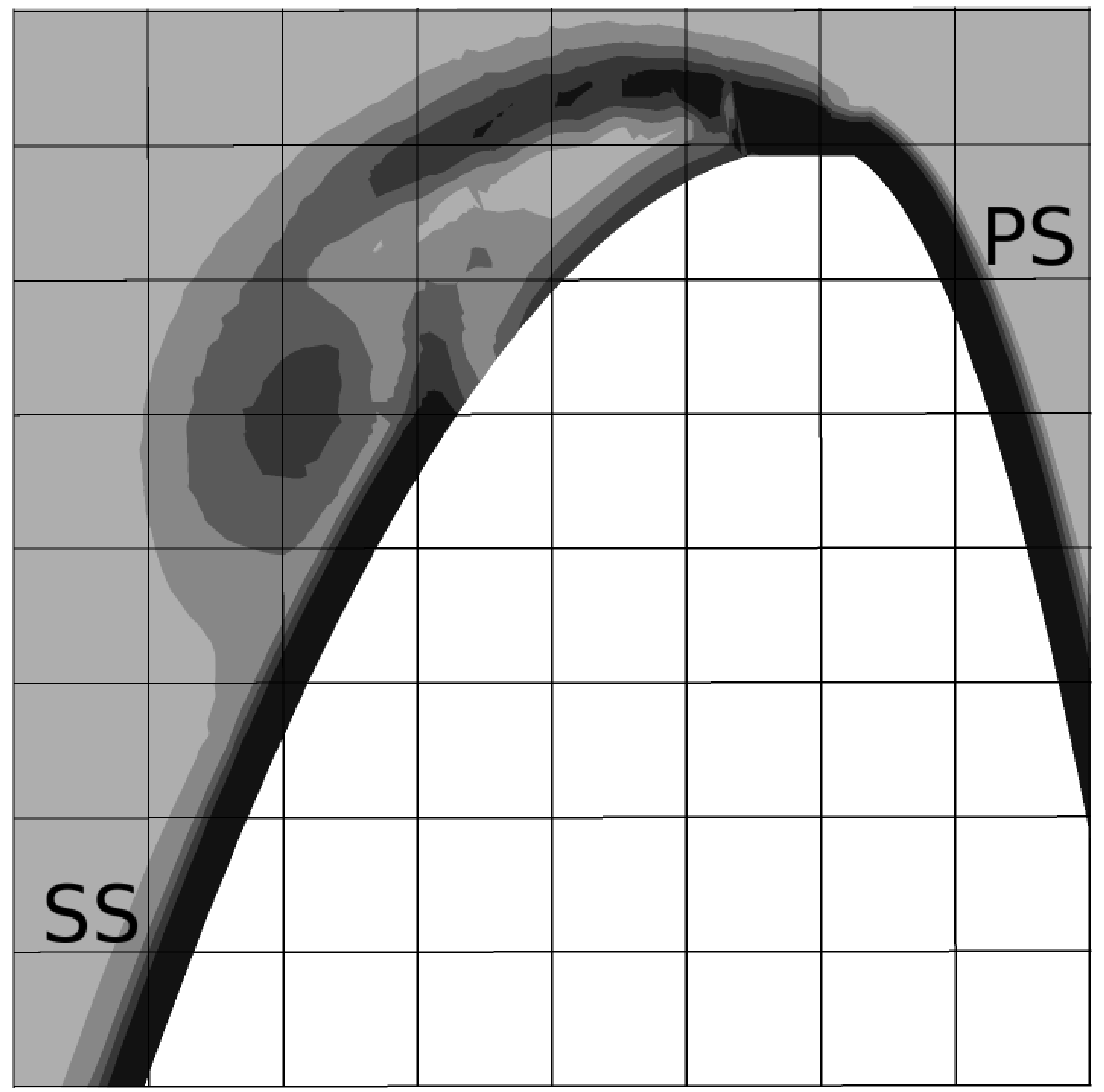




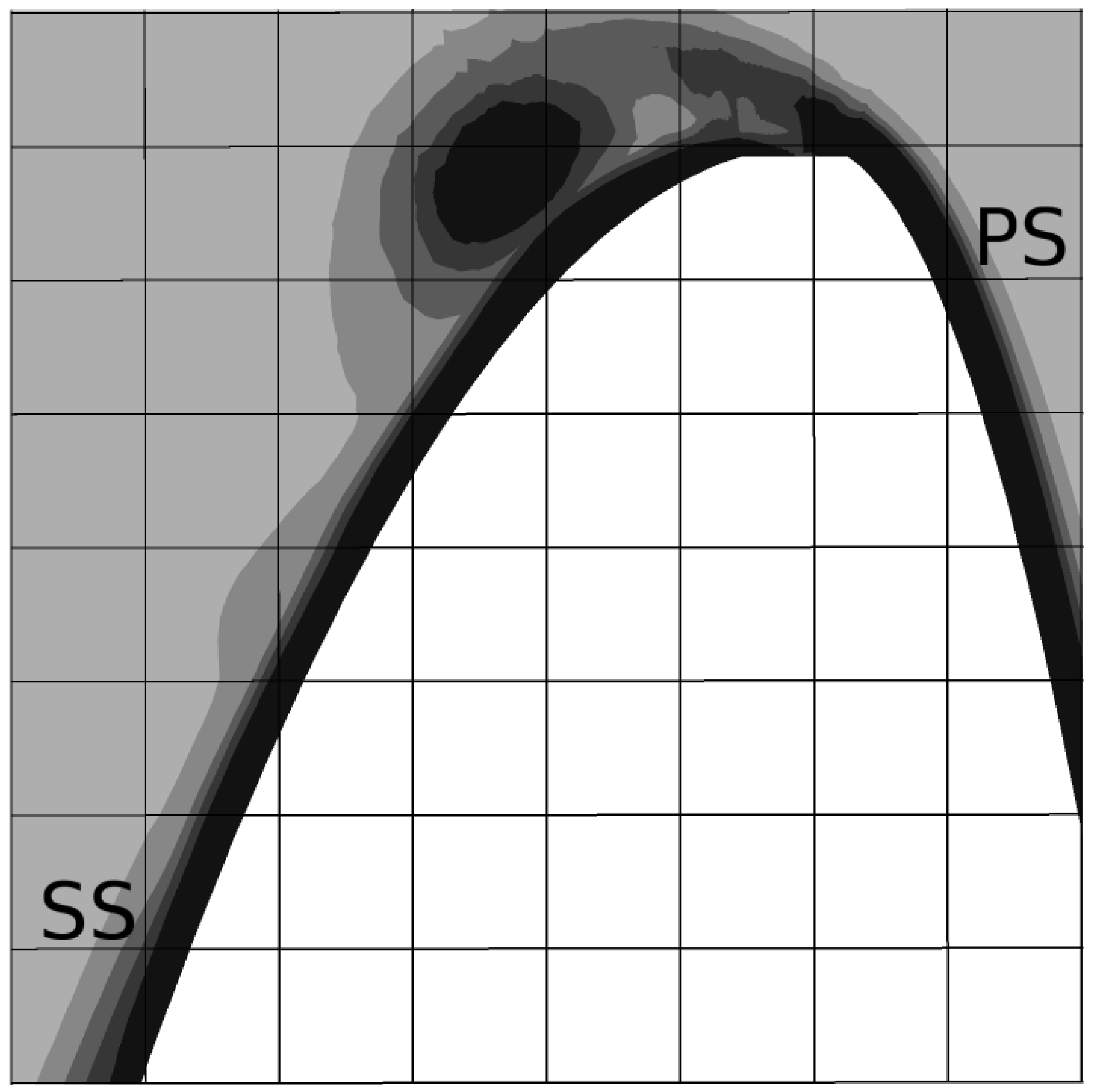




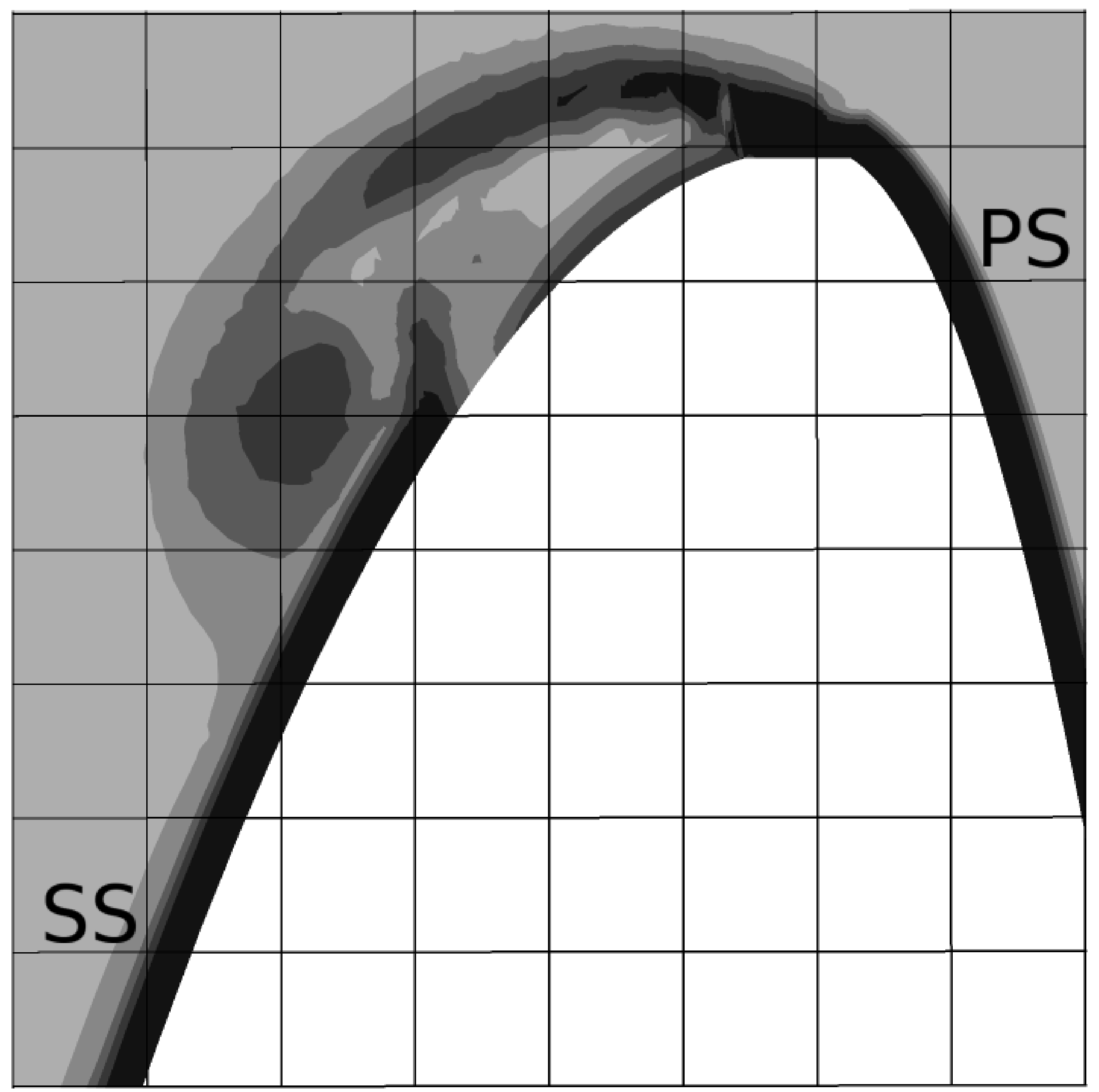




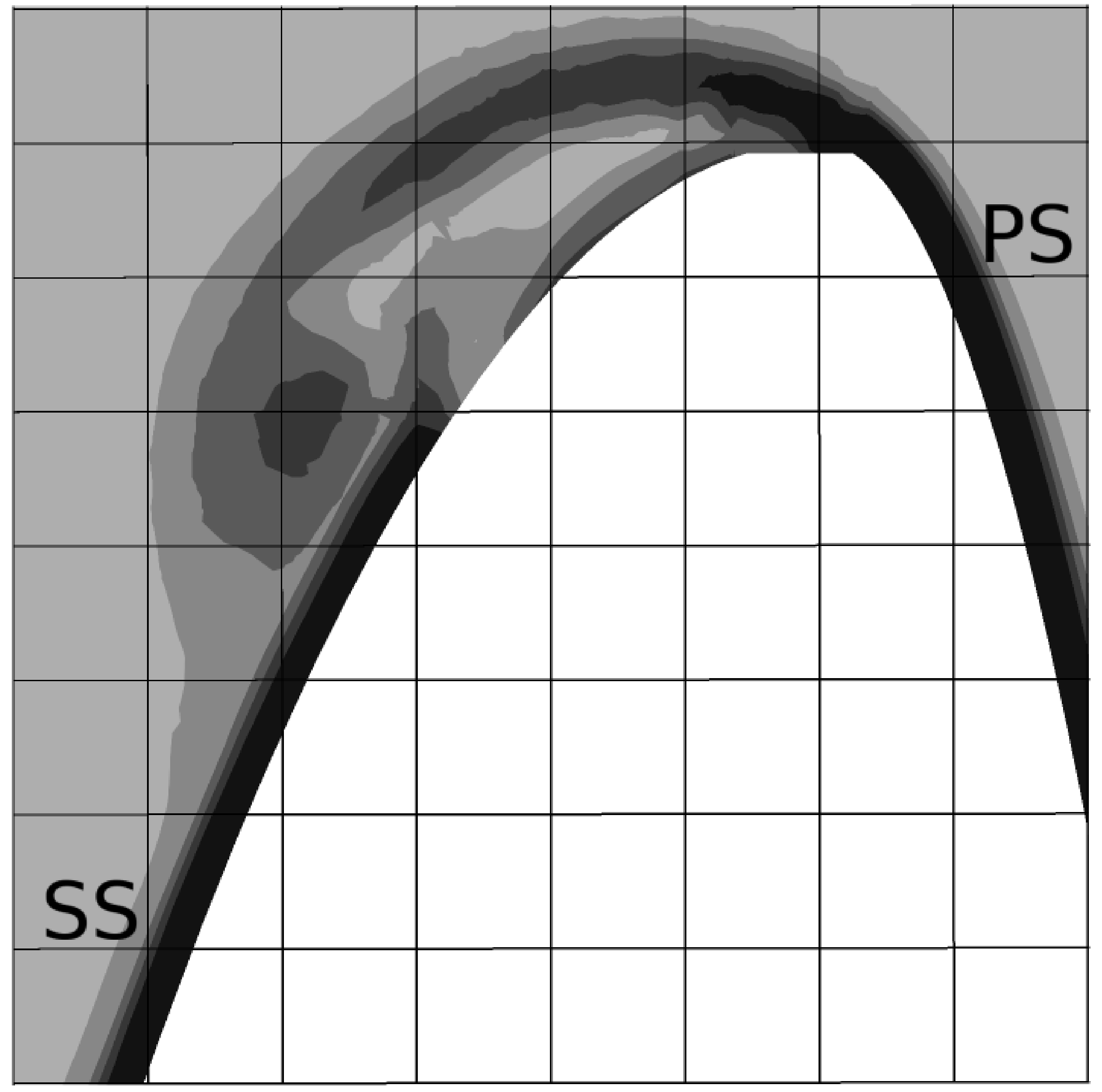




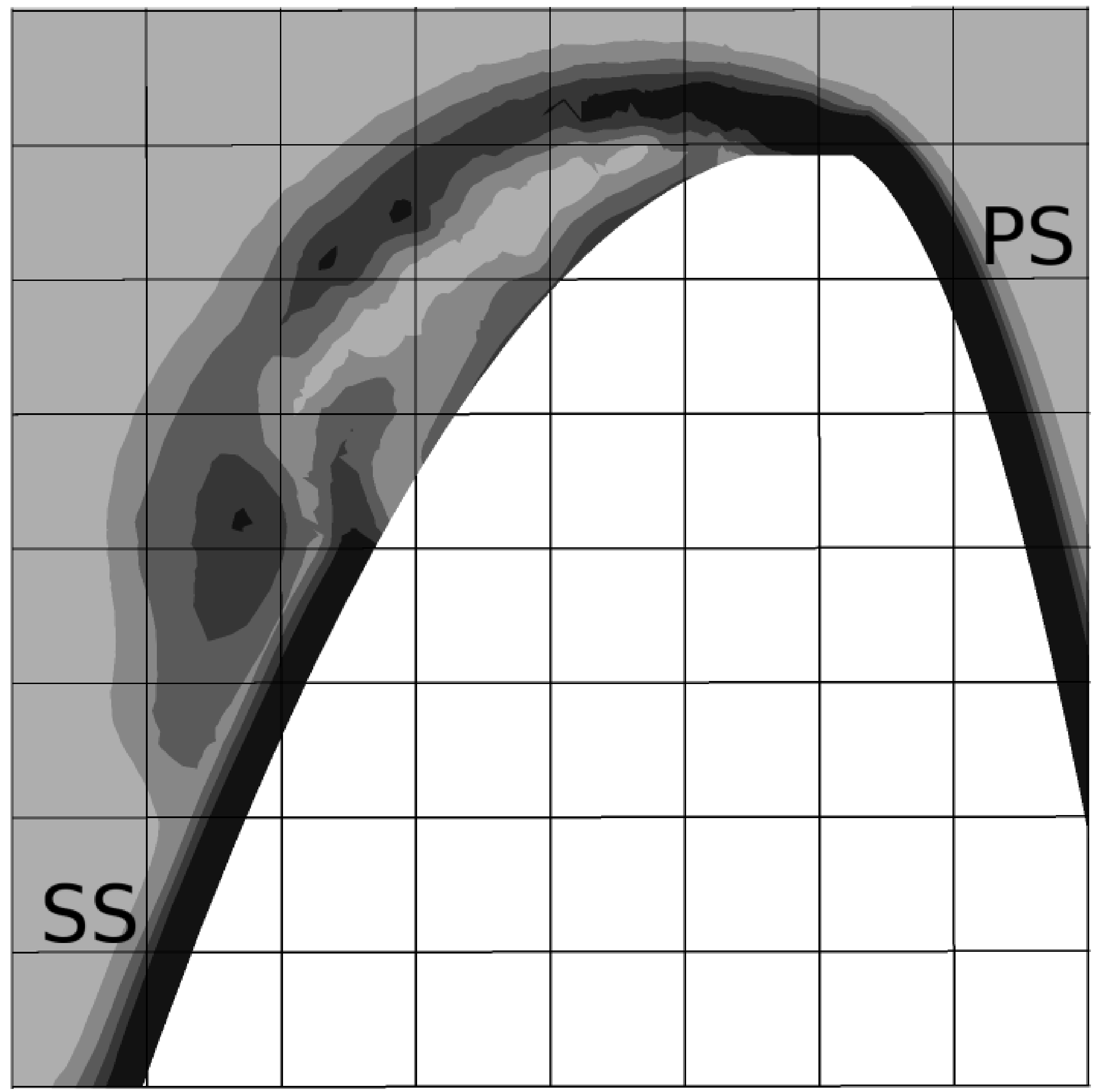




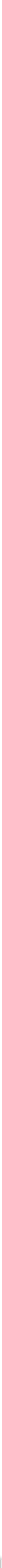




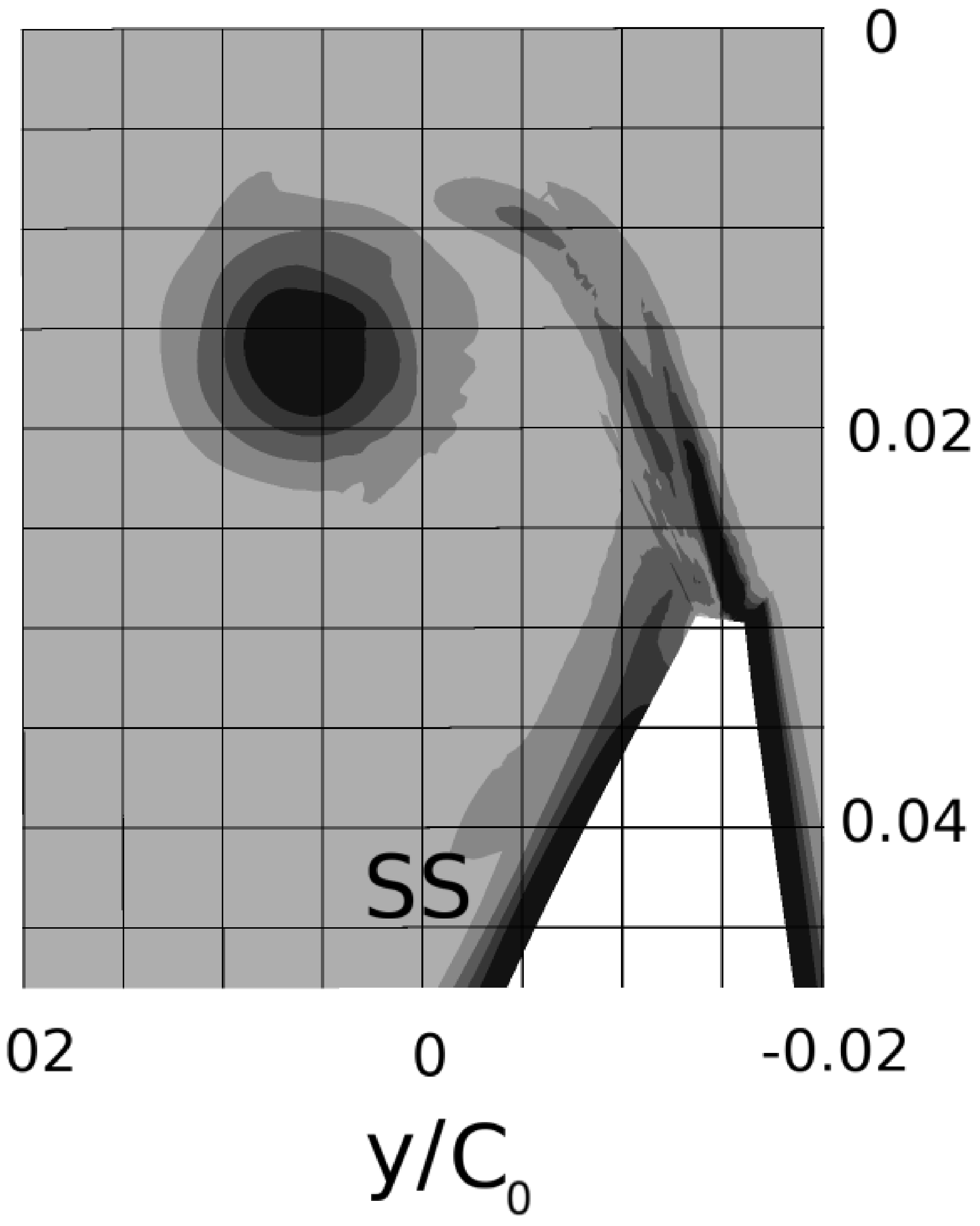

0.02 


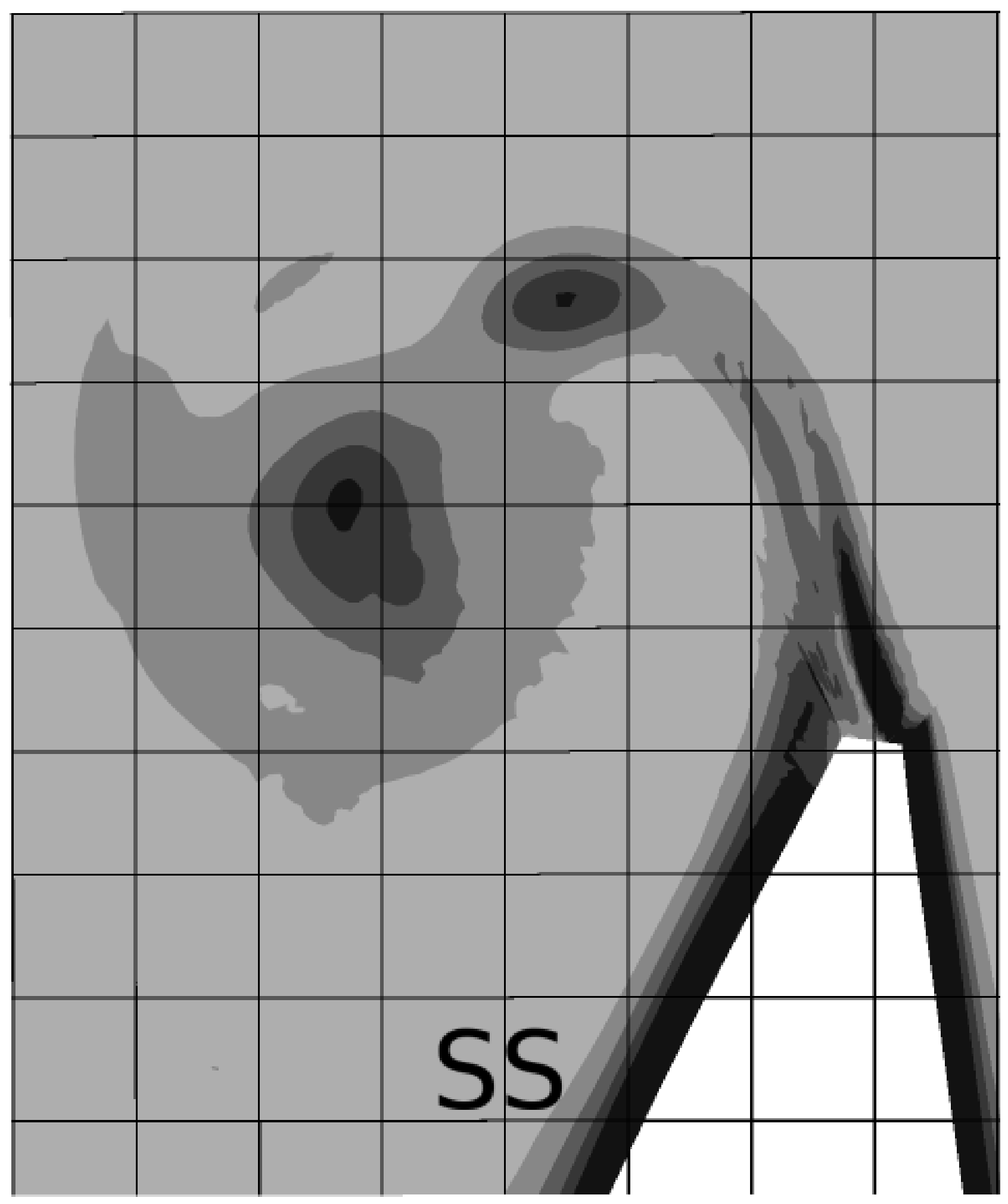




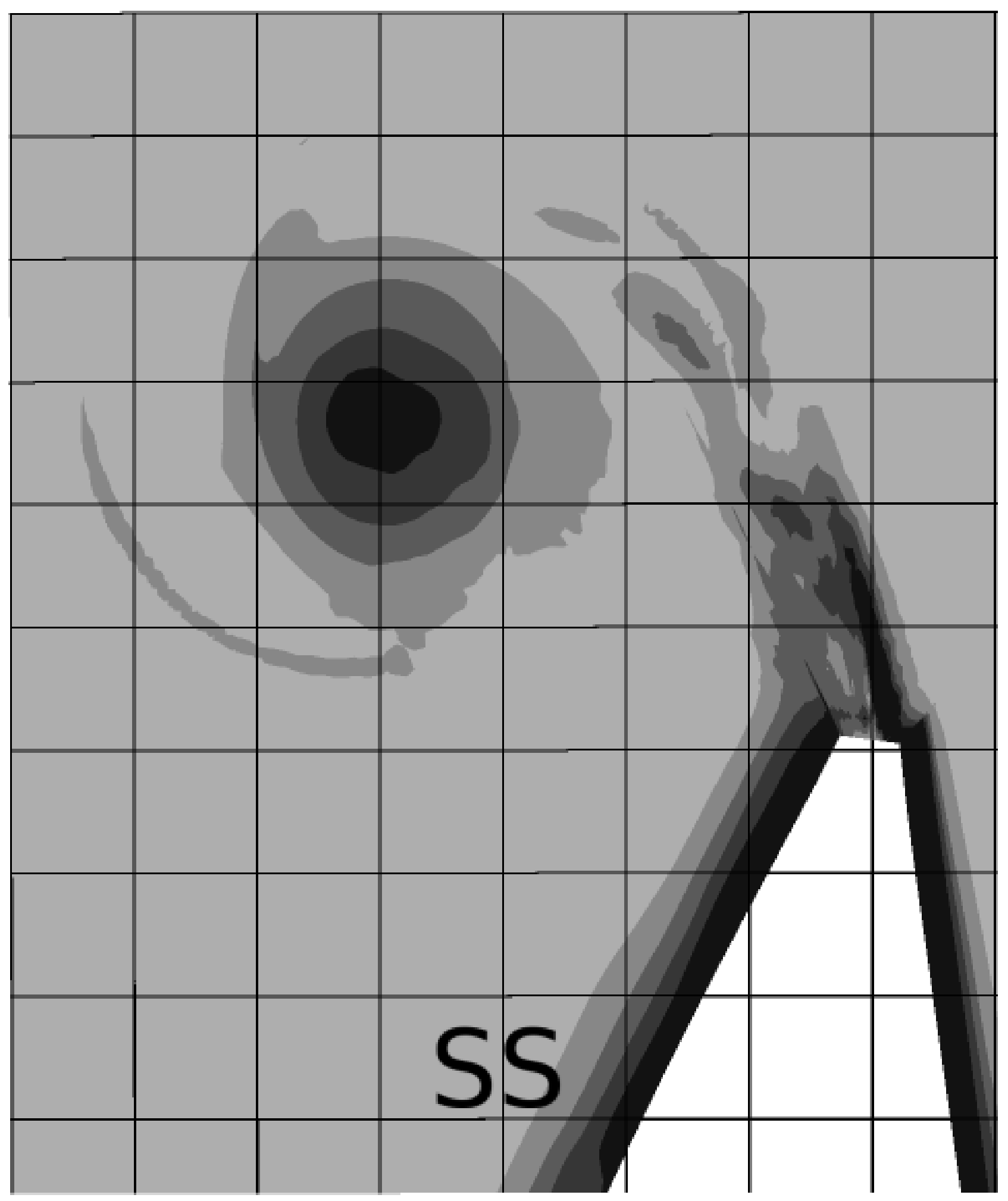




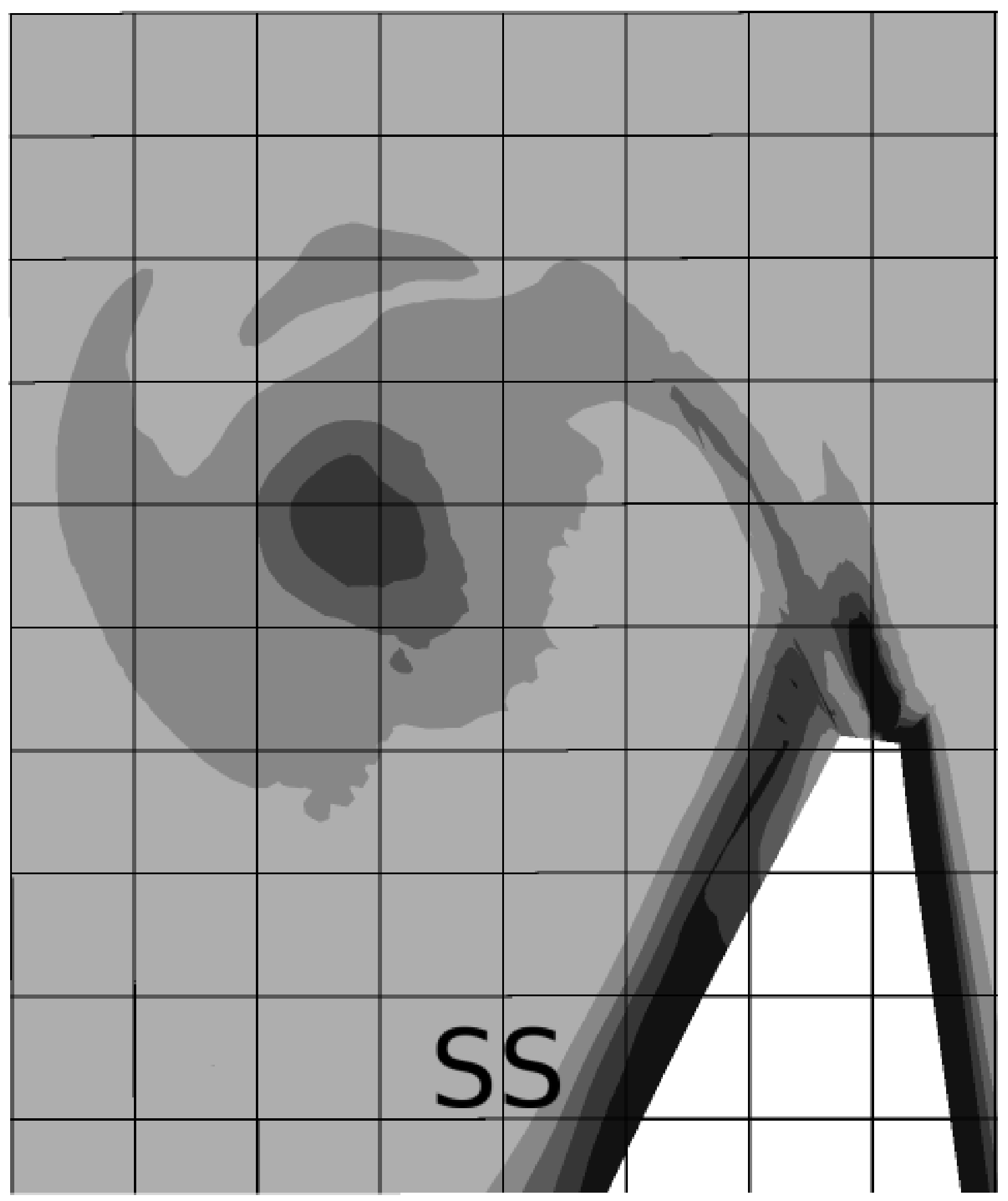




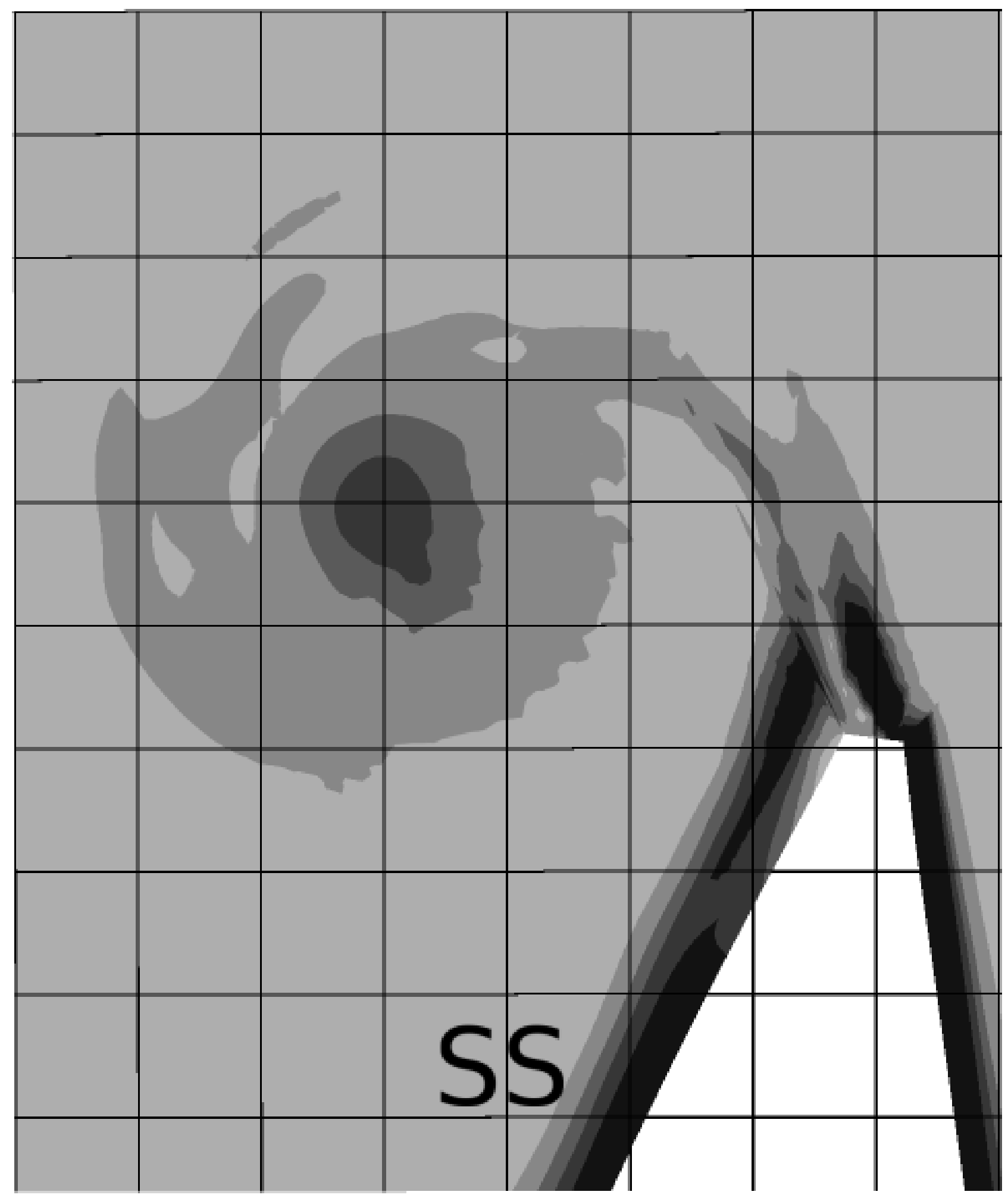


Magnitude of vorticity

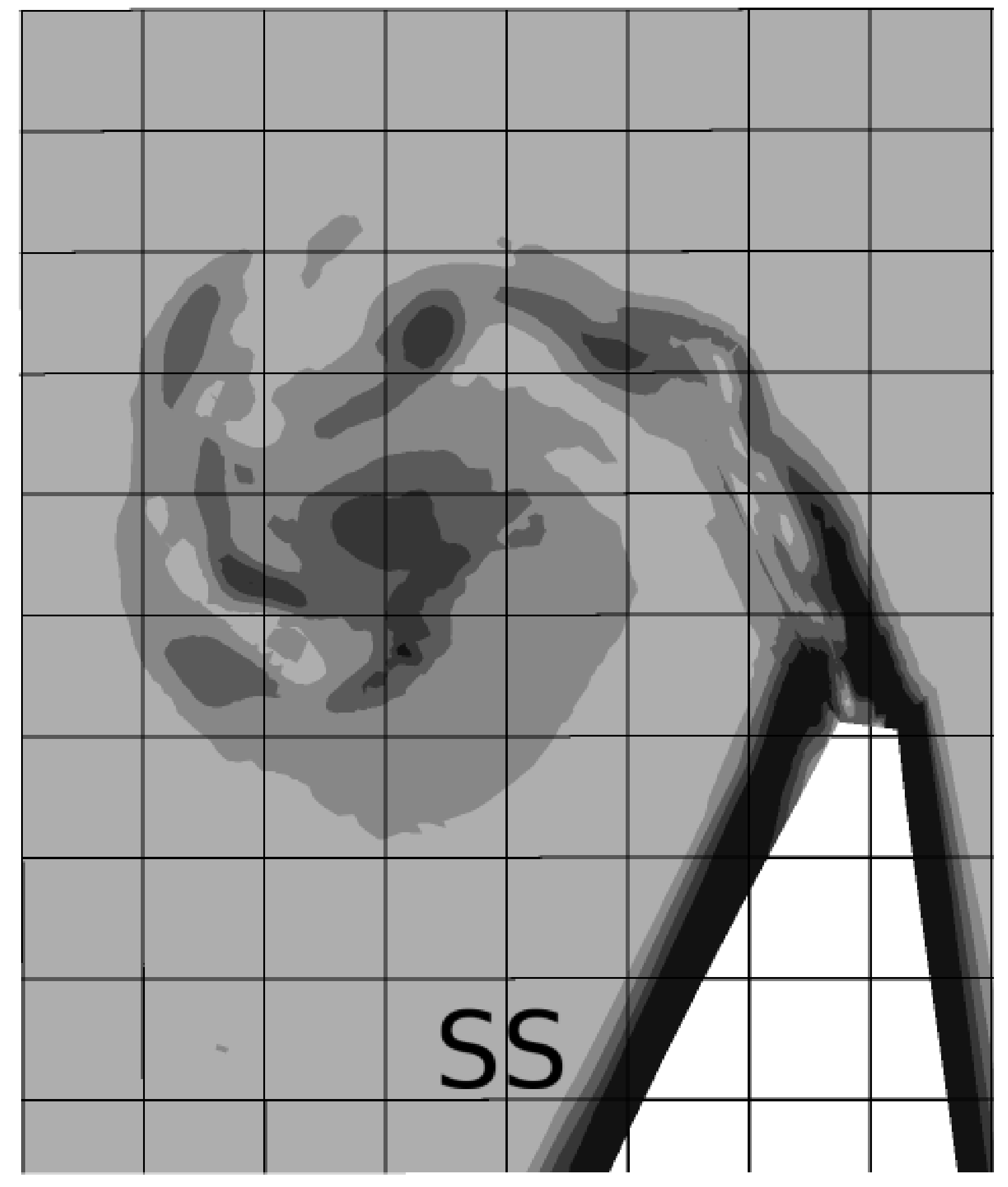

$3 \mathrm{E}+04$

$0 \mathrm{E}+00$ 


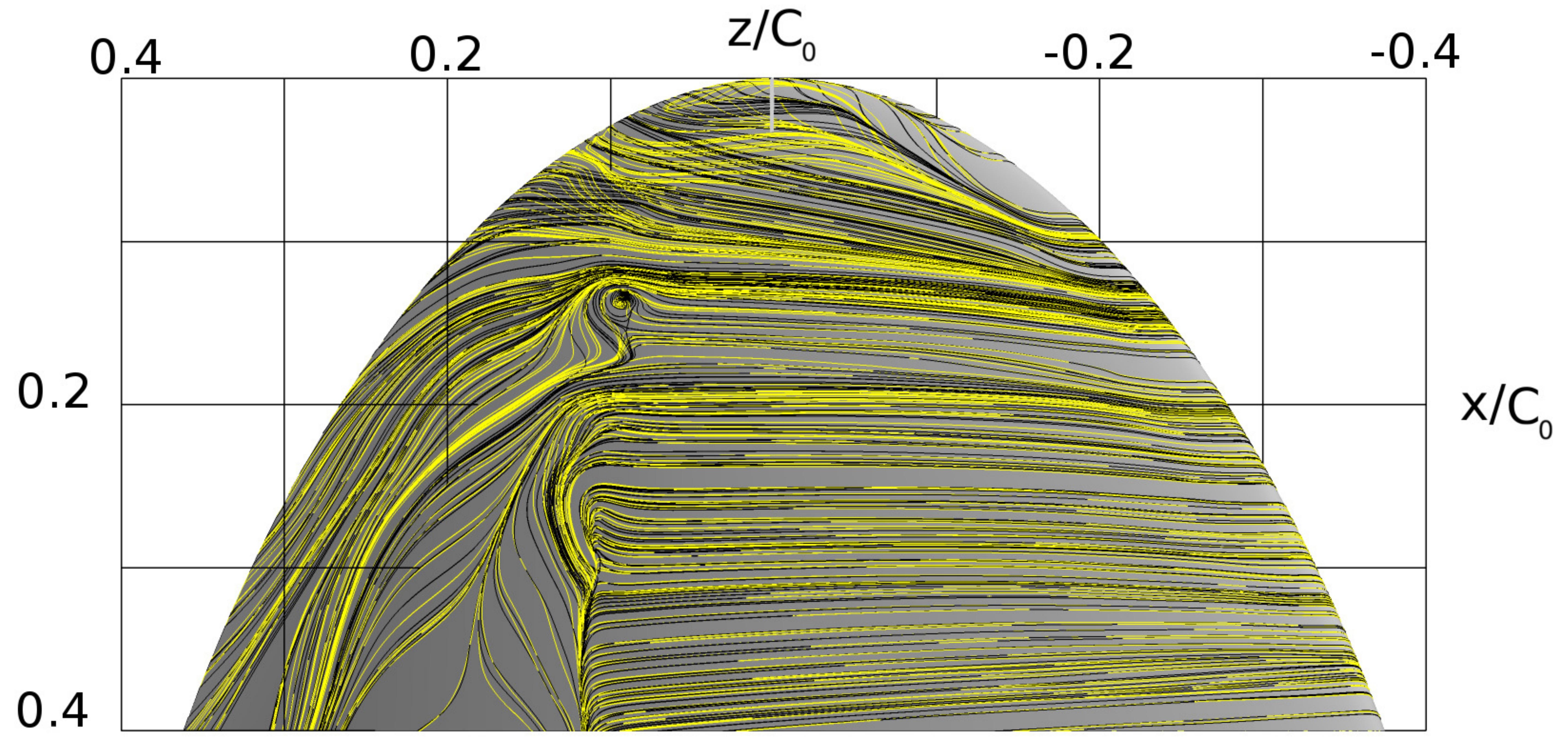




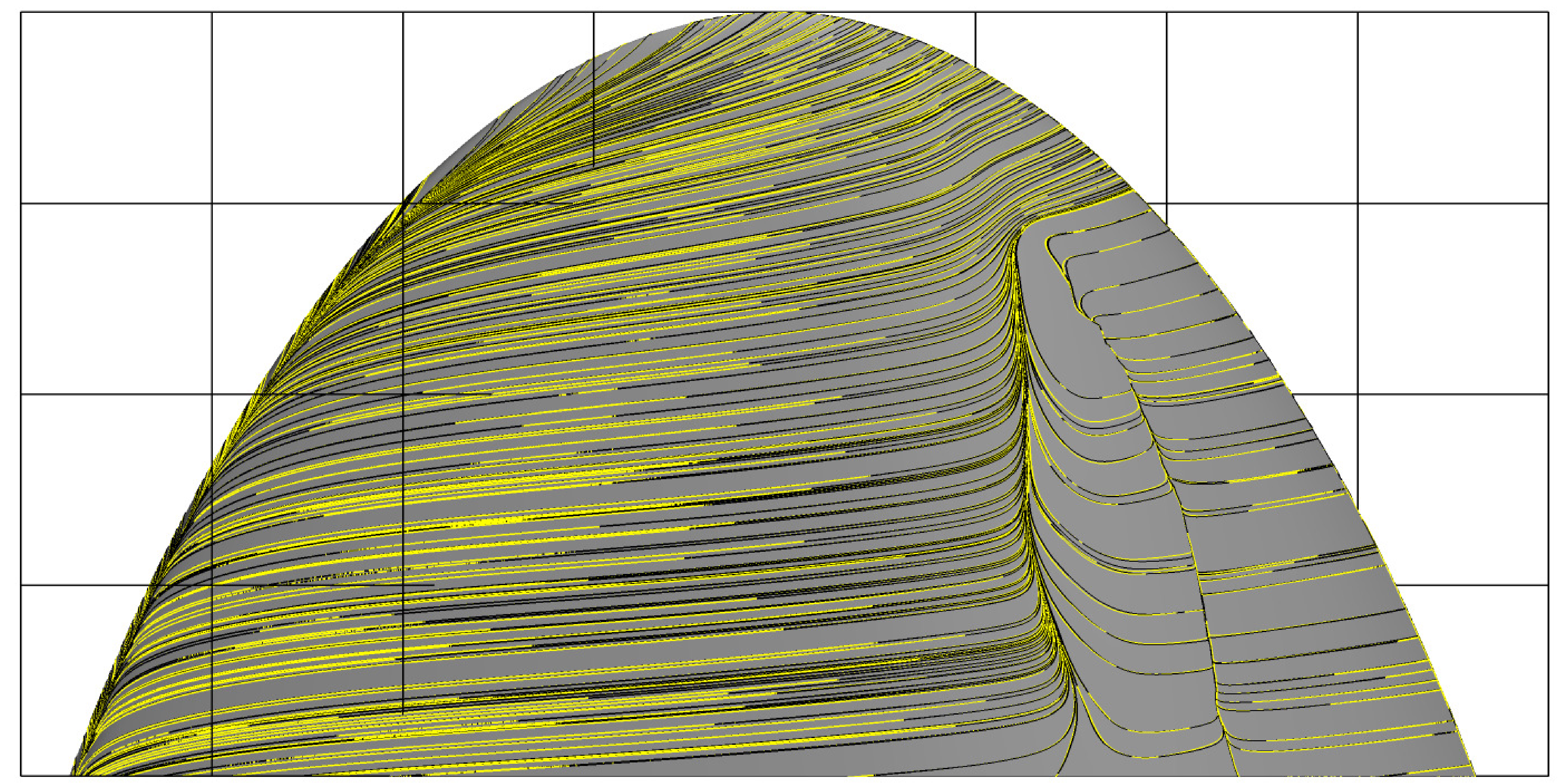



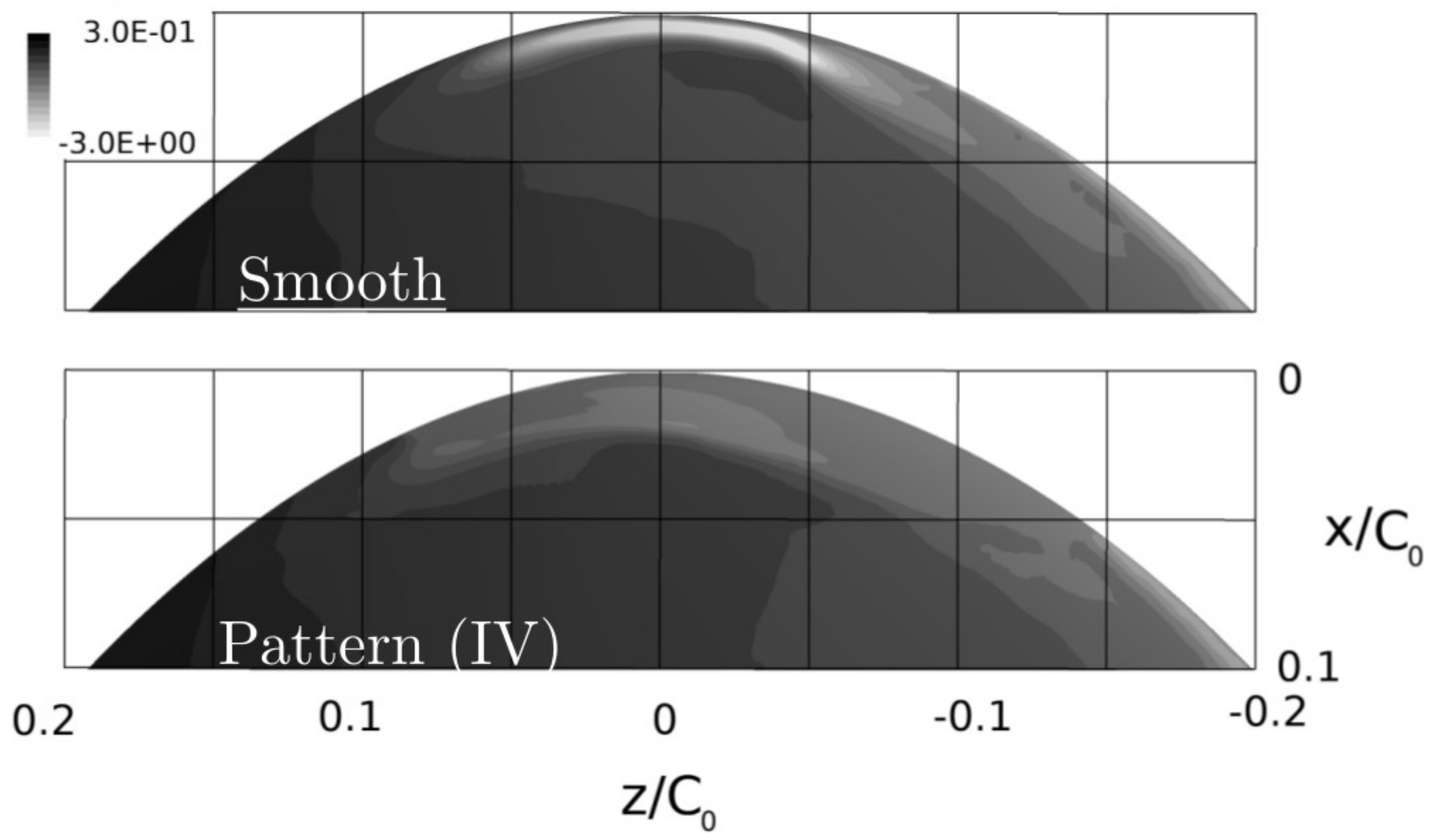
Roughness separation line

Leading edge separation lines
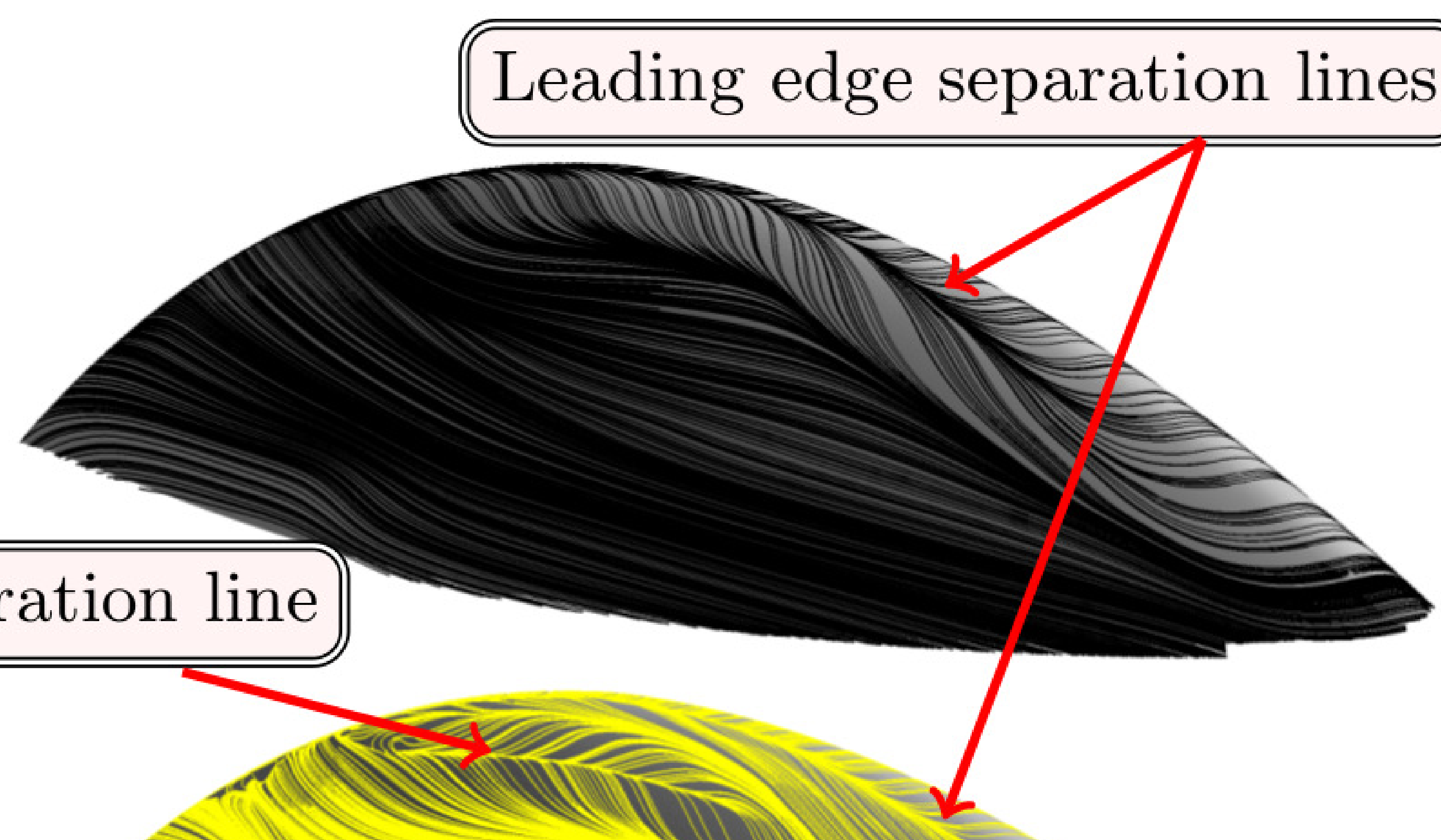

\section{,}




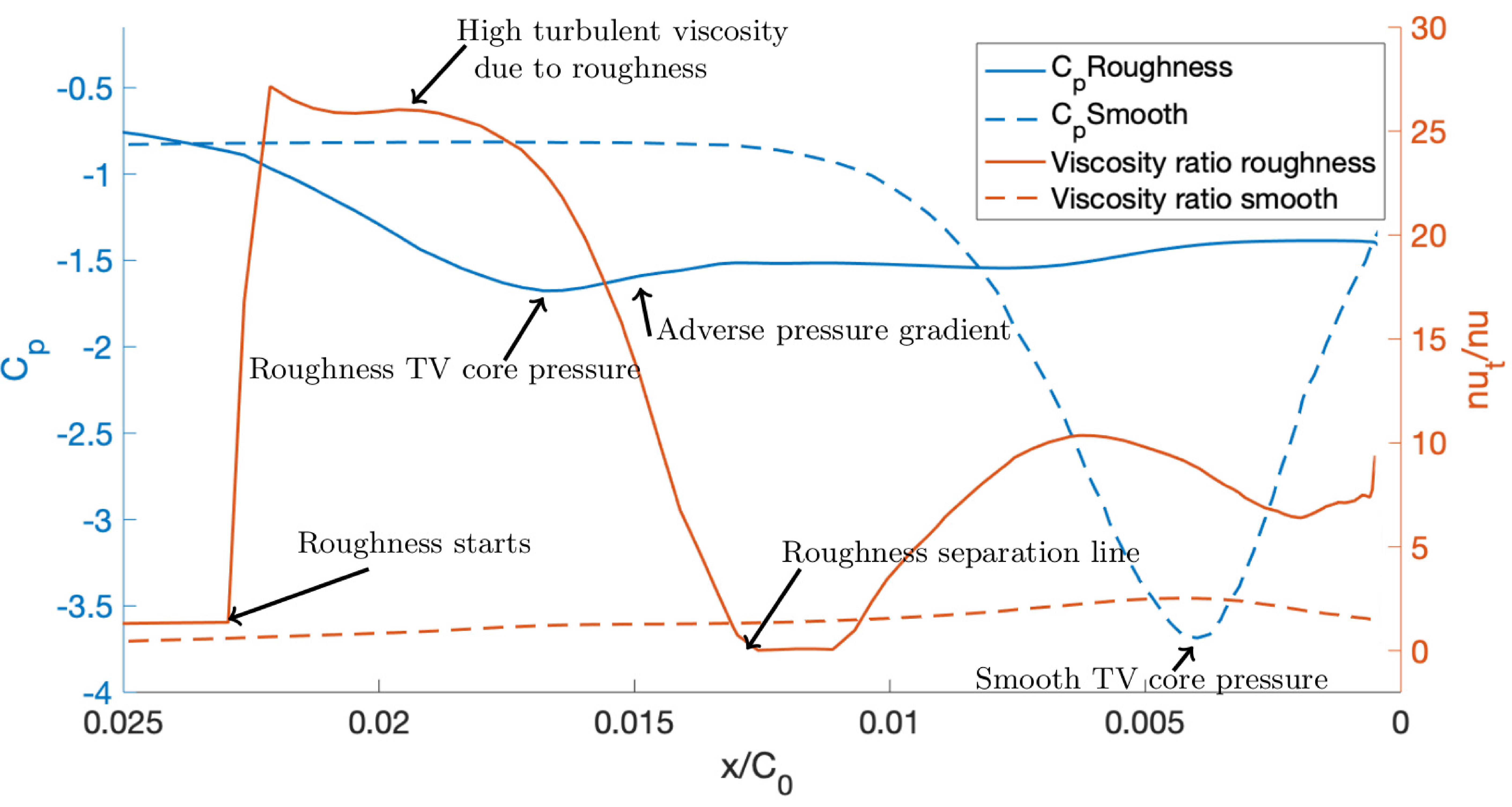




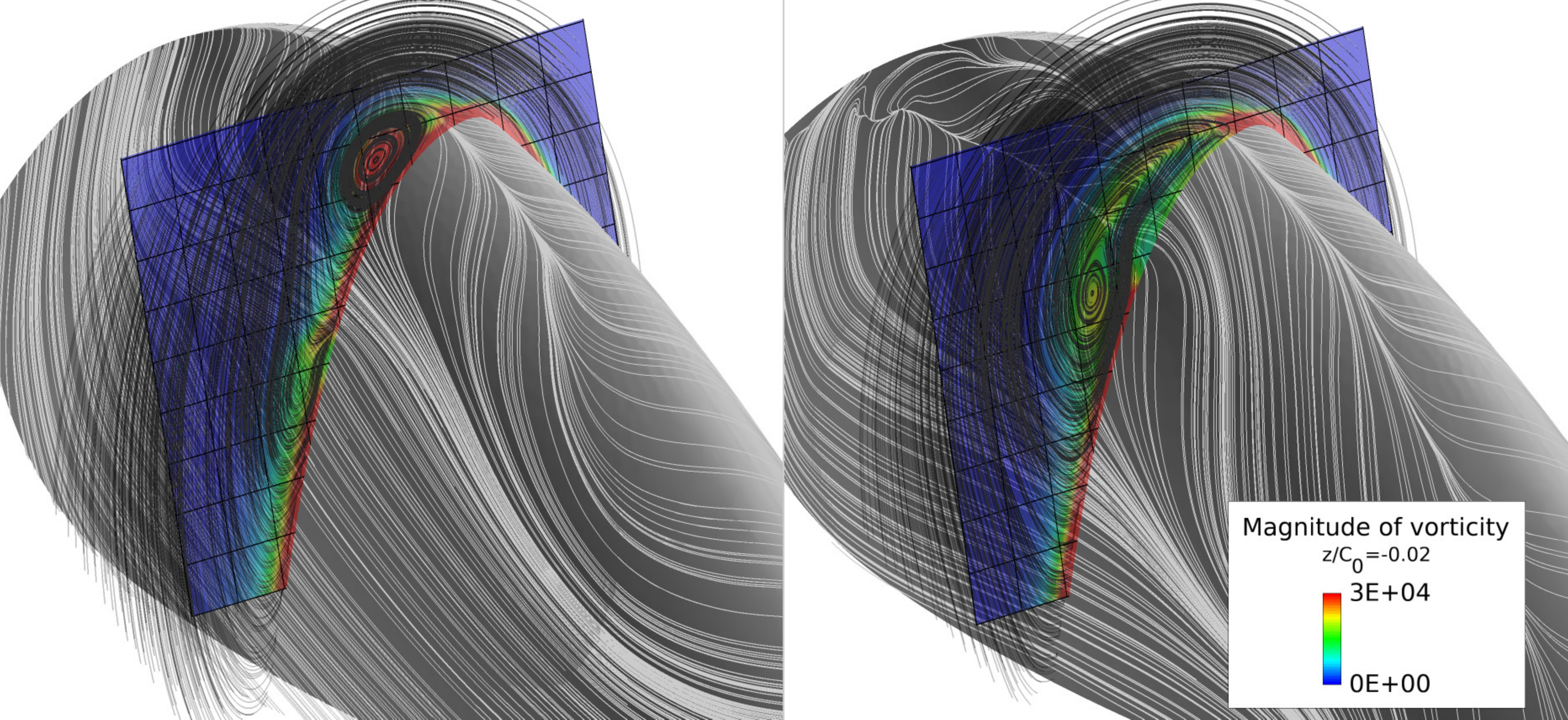



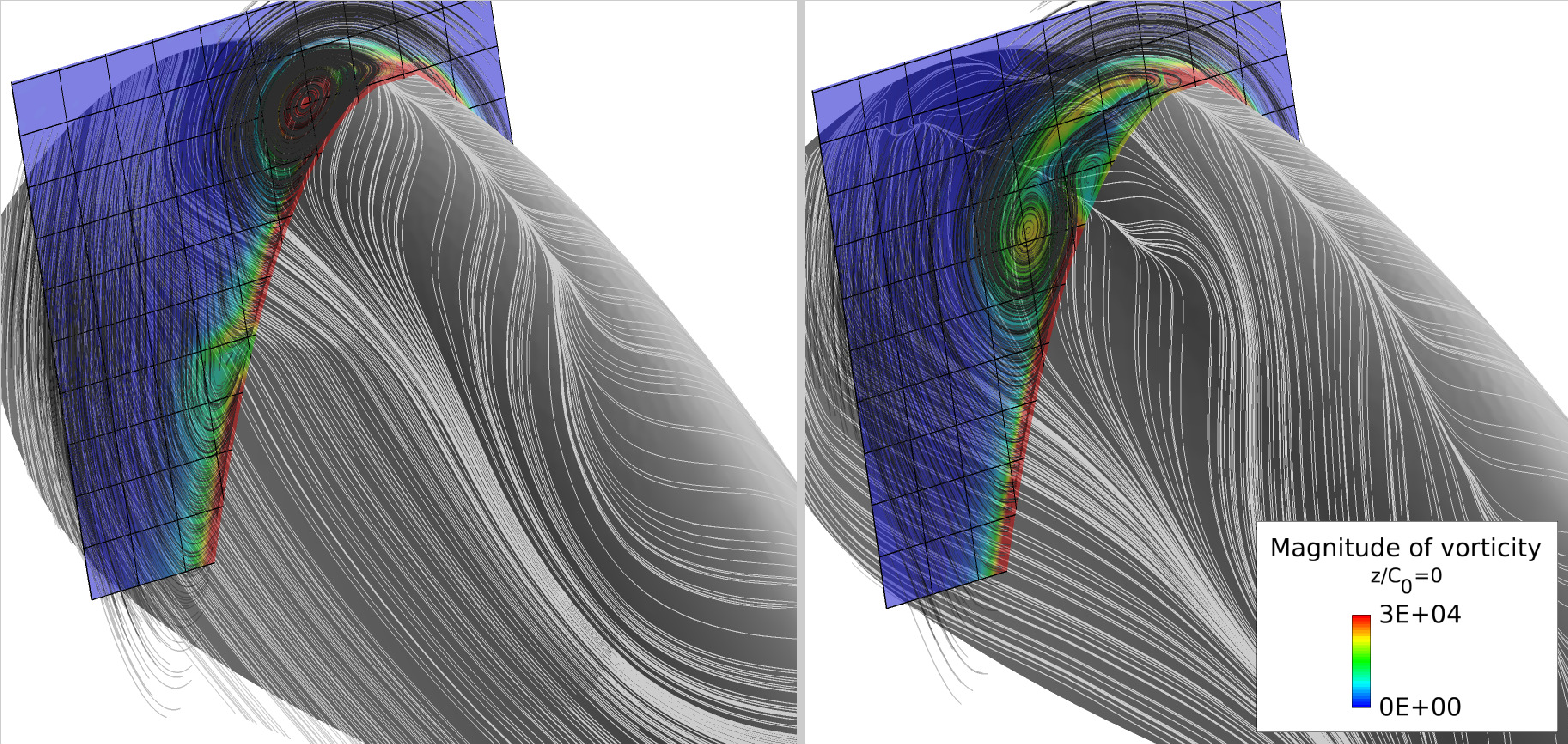

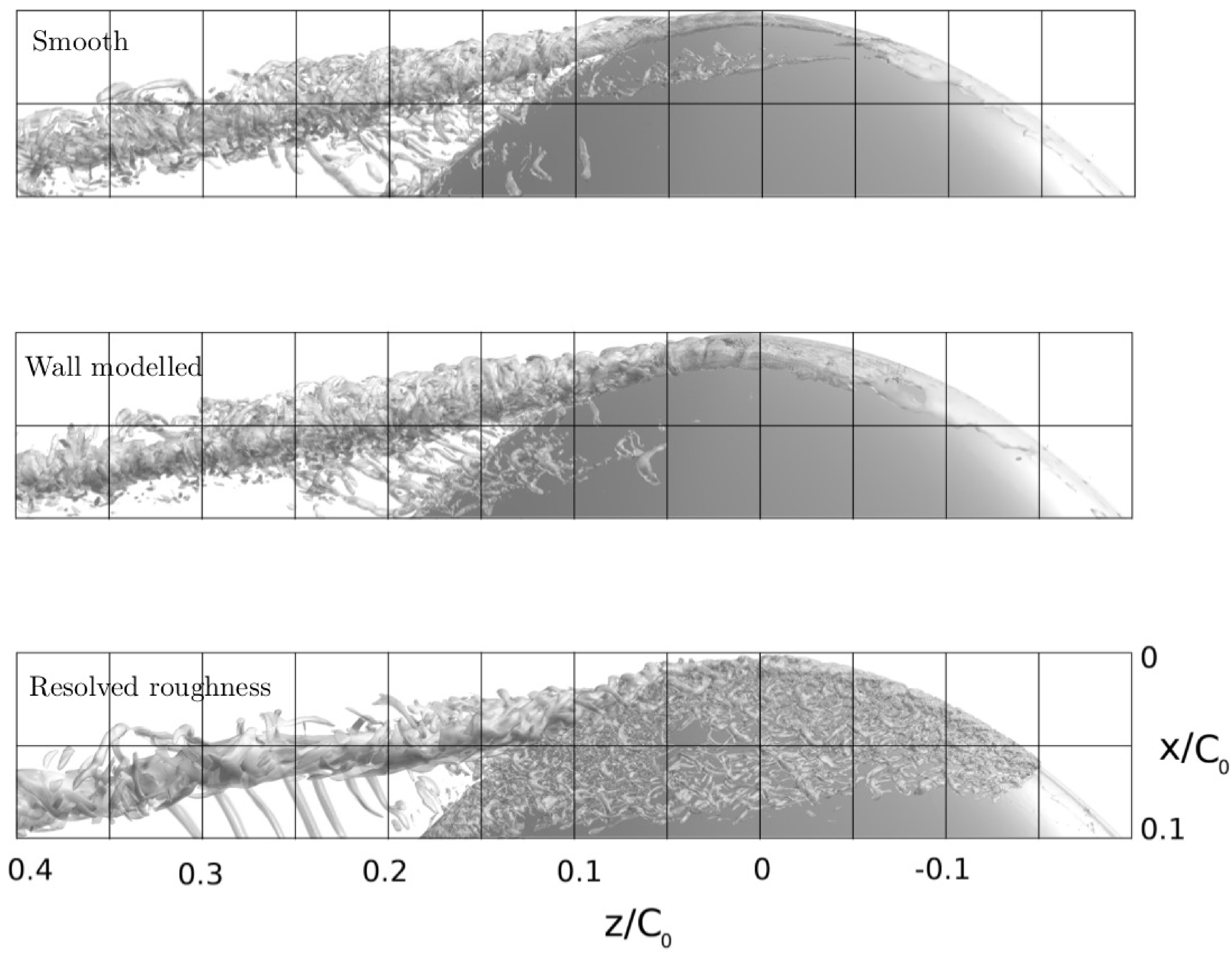


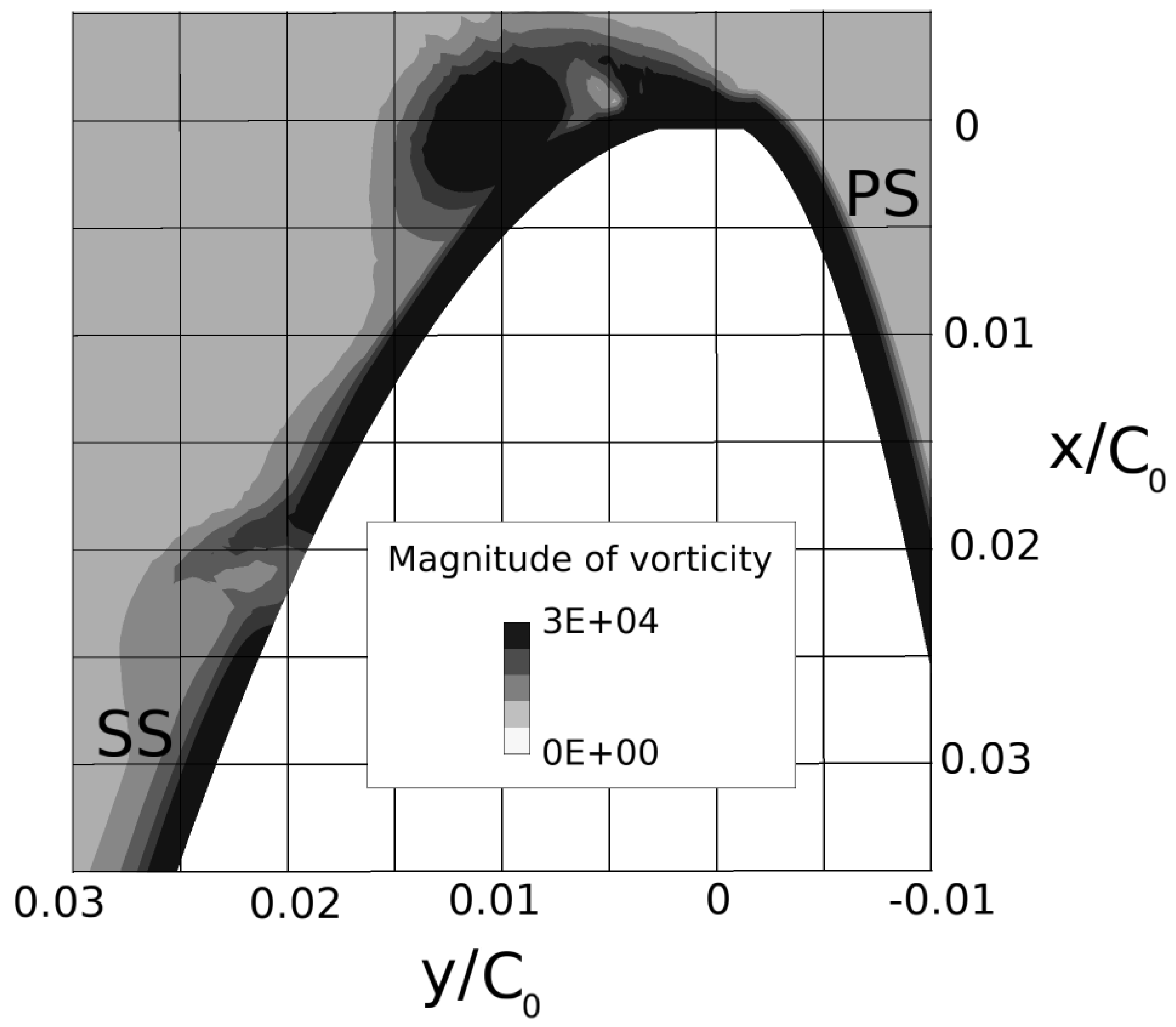




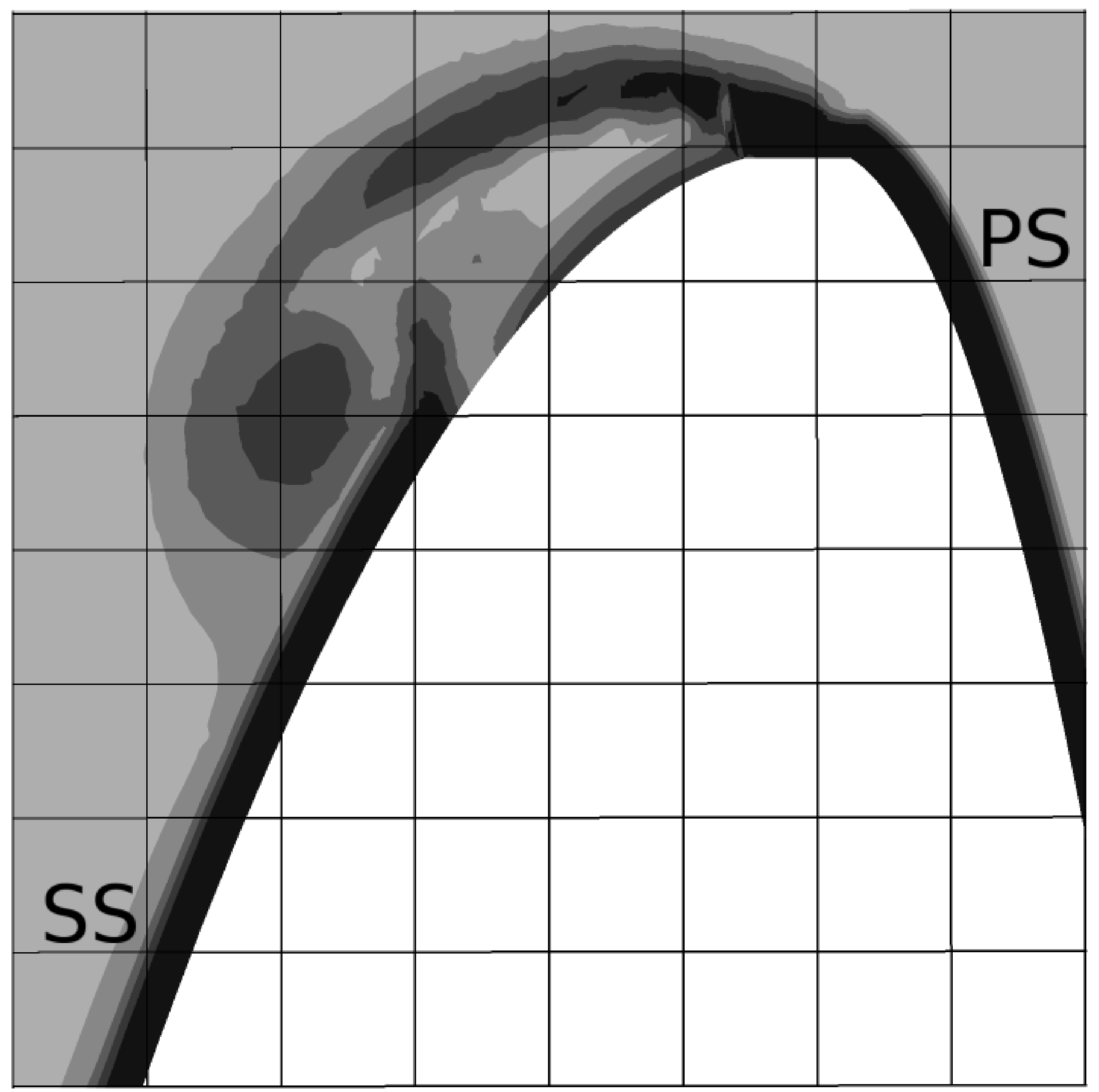




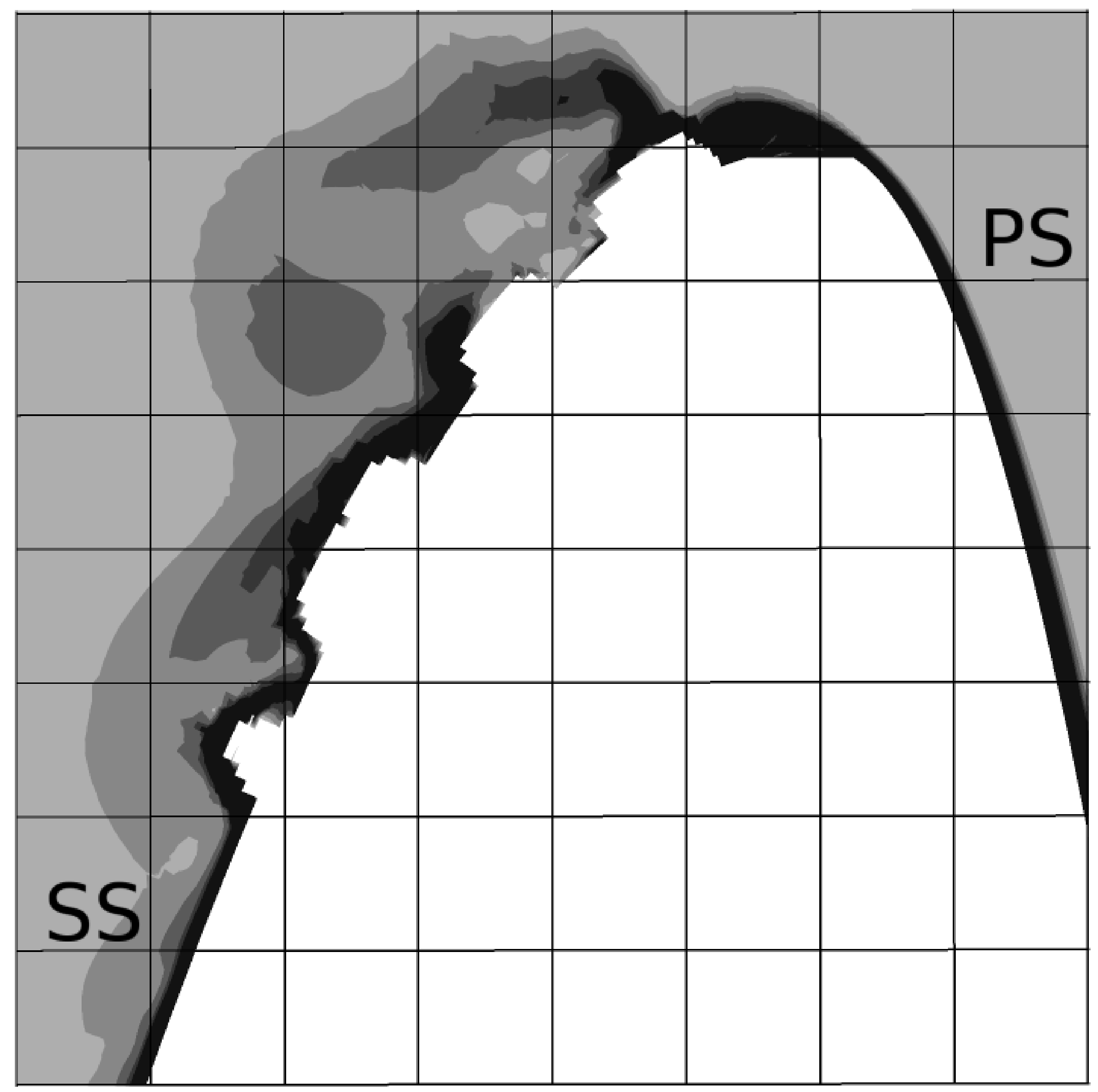




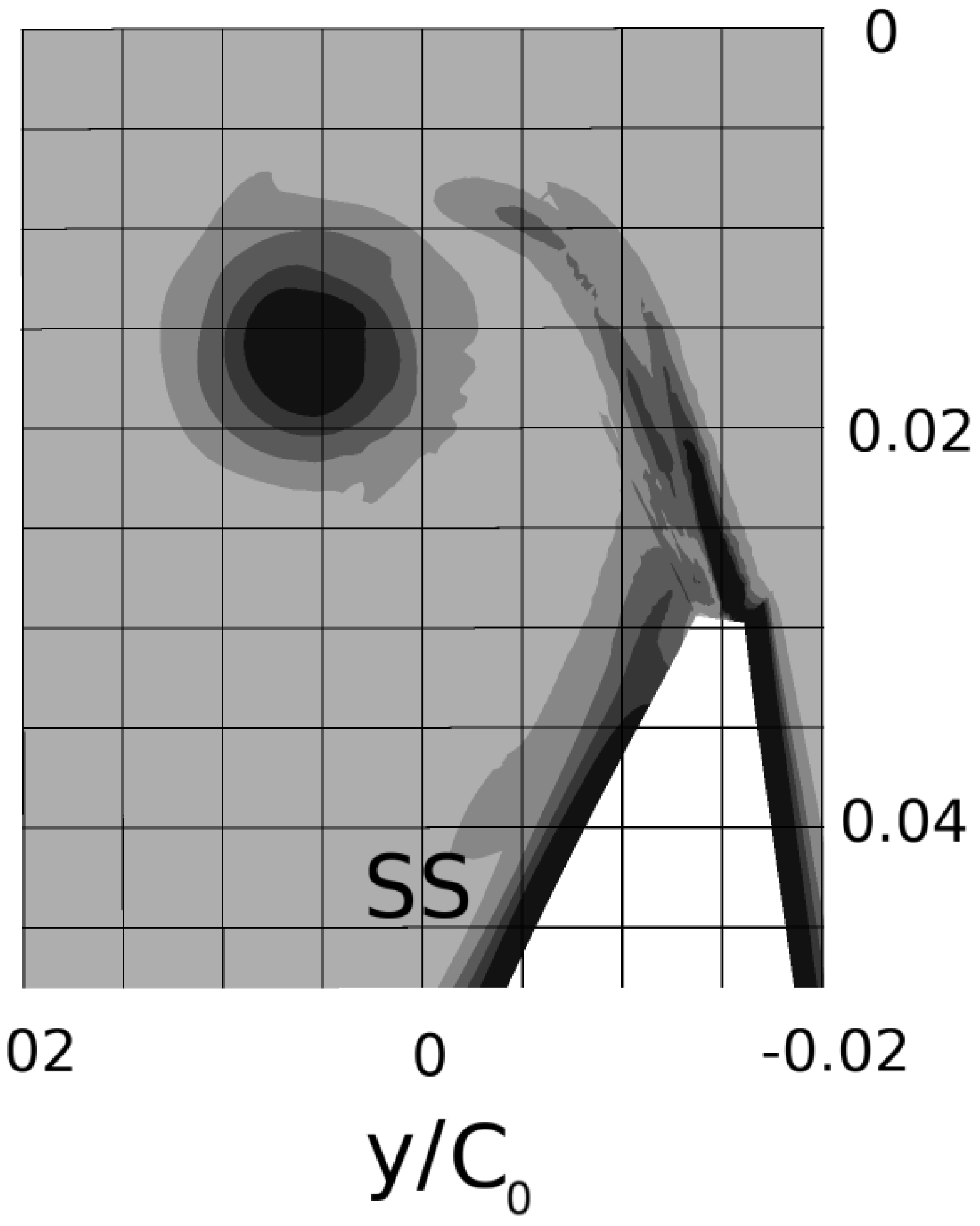

0.02 


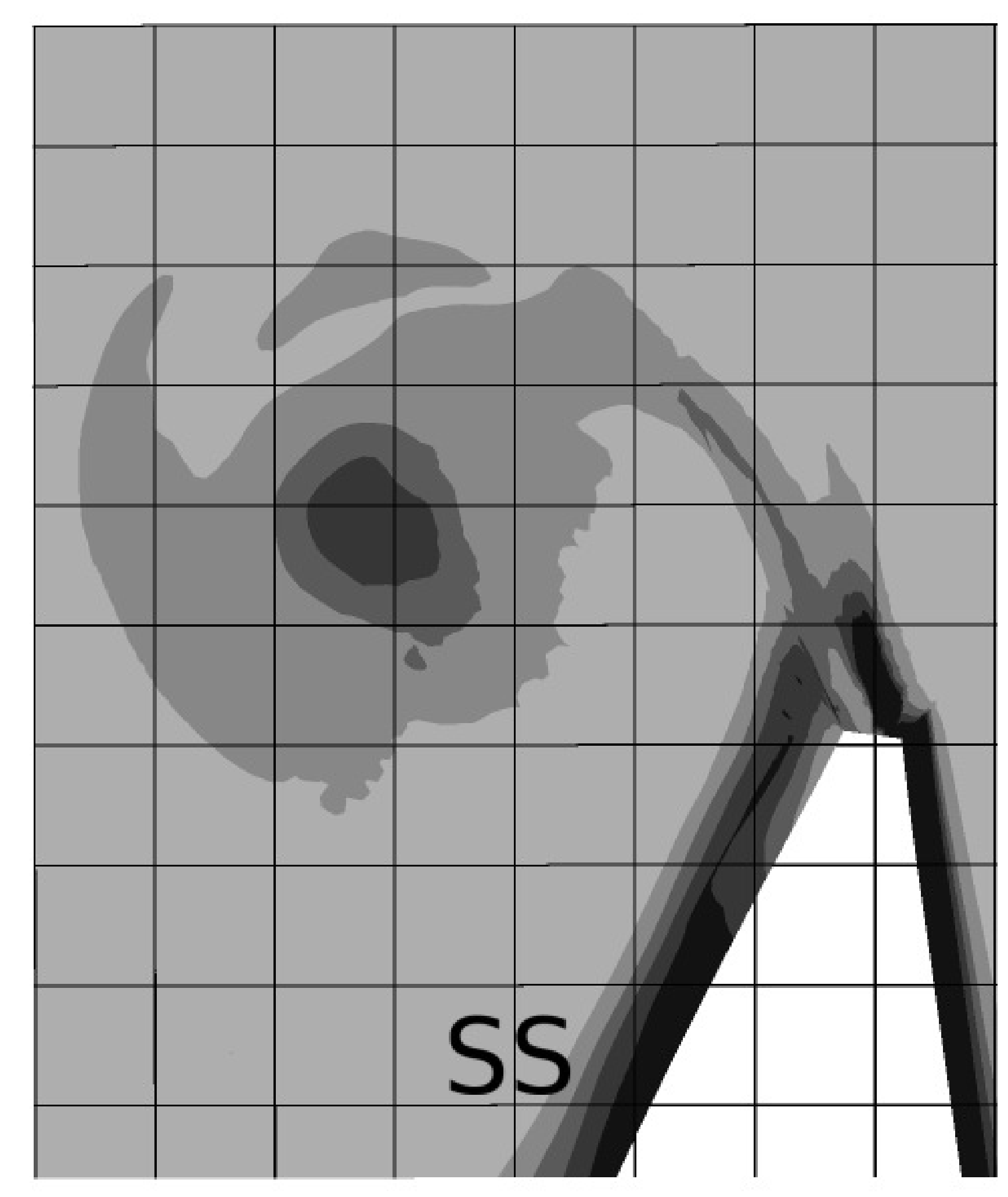




$$
9
$$


$-$.

. 



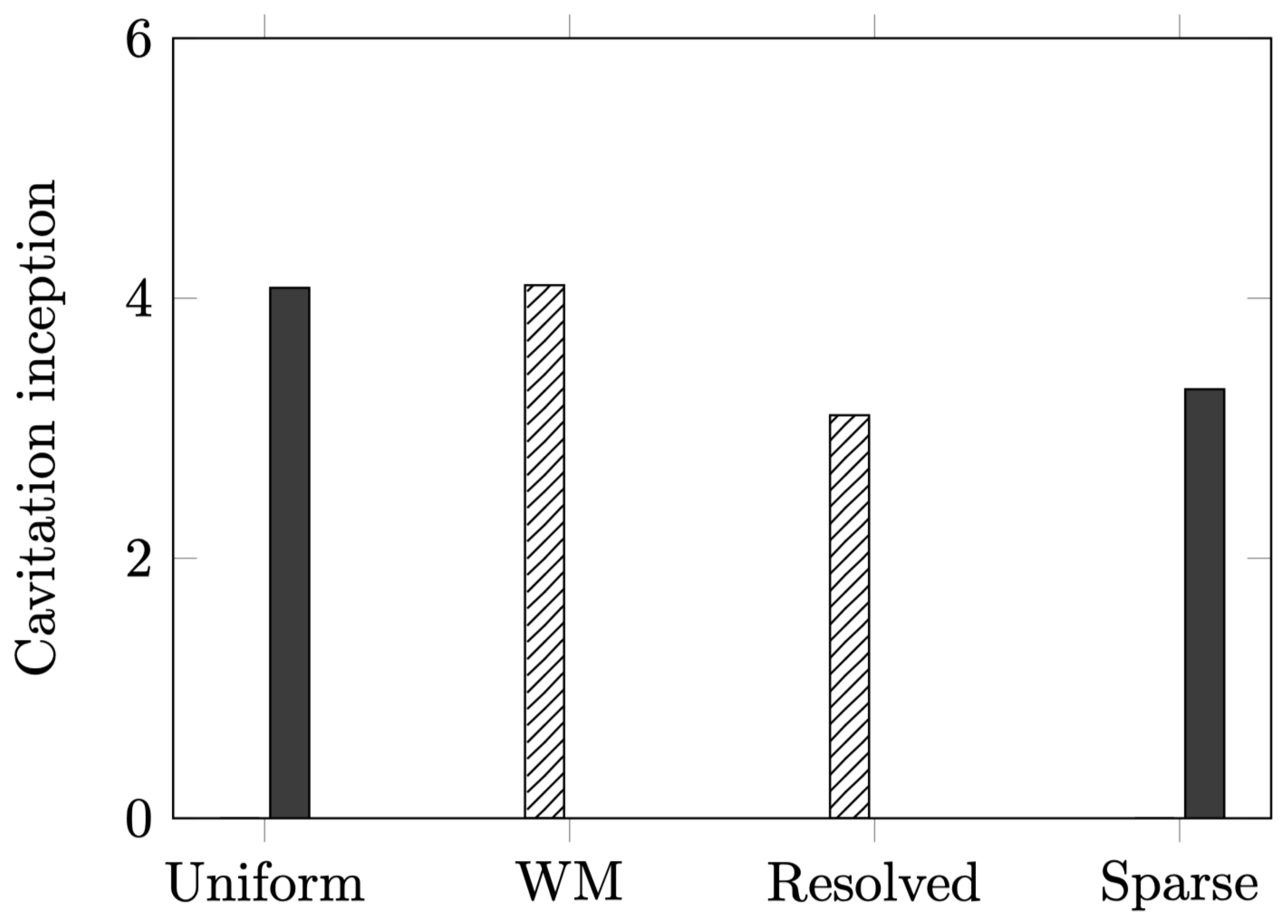




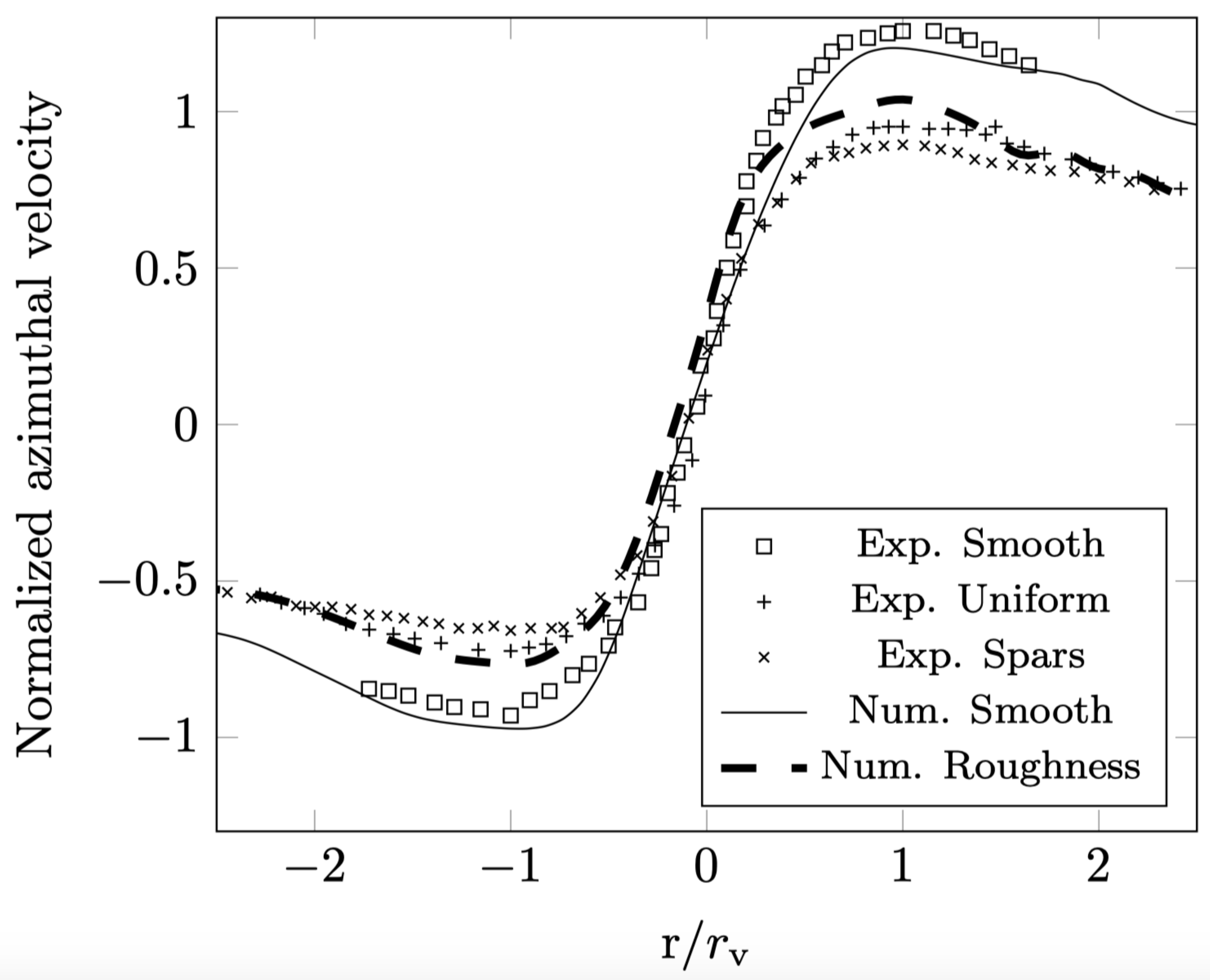




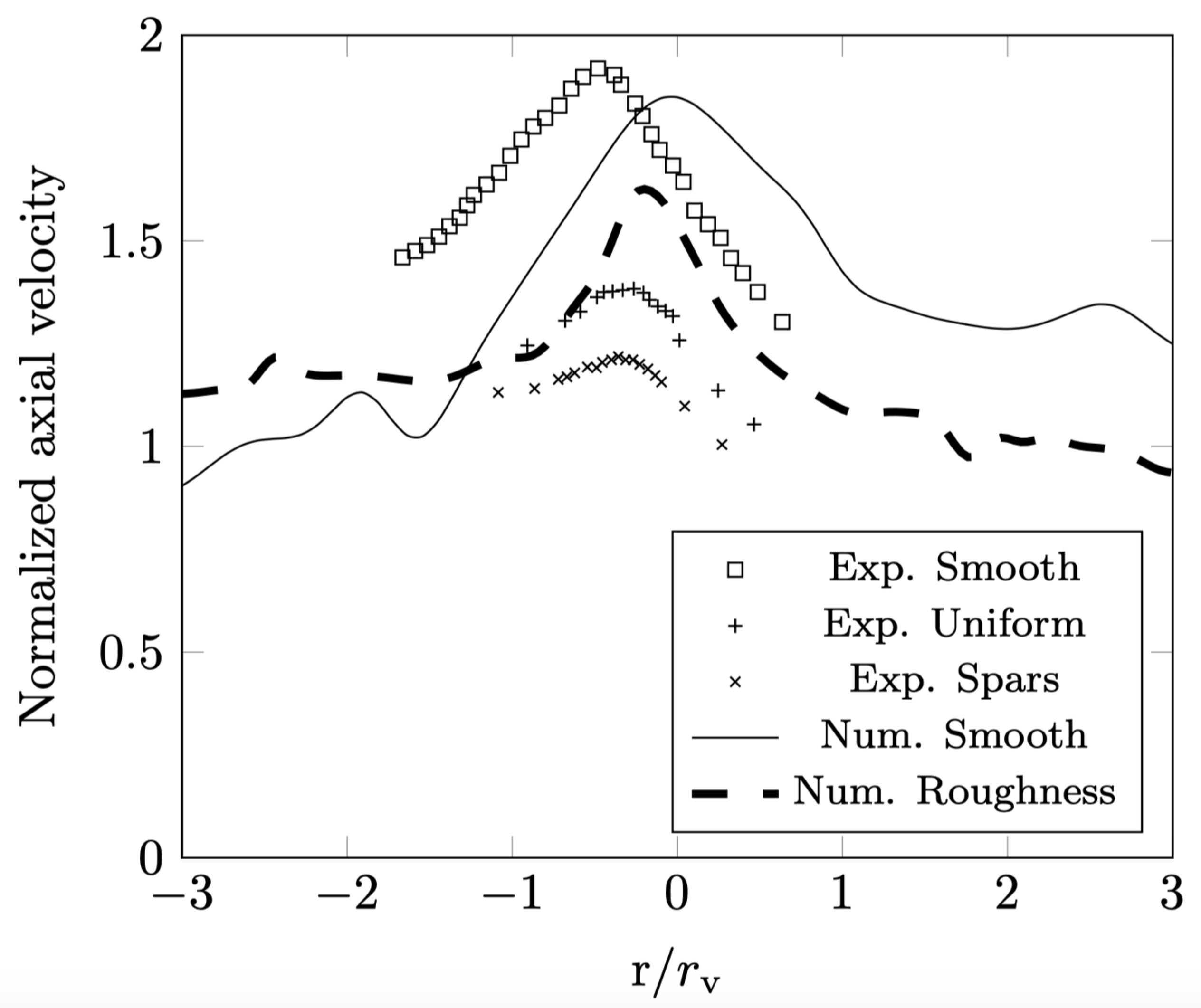

